\title{
Multi-Physics Simulations for Molten Salt Reactor Evaluation: Chemistry Modeling and Database Development
}

\section{Approved for public release.} Distribution is unlimited.
J. McMurray ${ }^{1}$
T. Besmann ${ }^{2}$
J. Jerden ${ }^{3}$
M. Williamson ${ }^{3}$
J. Ard ${ }^{2}$
B. Fitzpatrick ${ }^{4}$
M. Piro ${ }^{4}$
A. Graham ${ }^{1}$
Z. Taylor ${ }^{1}$
B. Collins ${ }^{1}$
B. Betzler ${ }^{1}$
L. Qualls ${ }^{1}$
T. Pandya ${ }^{1}$
S. Greenwood ${ }^{1}$
S. Johnson ${ }^{1}$

1. Oak Ridge National Laboratory

2. University of South Carolina

3. Argonne National Laboratory 4. University of Ontario Institute of Technology

June 2018 


\section{DOCUMENT AVAILABILITY}

Reports produced after January 1, 1996, are generally available free via US Department of Energy (DOE) SciTech Connect.

Website www.osti.gov

Reports produced before January 1, 1996, may be purchased by members of the public from the following source:

National Technical Information Service

5285 Port Royal Road

Springfield, VA 22161

Telephone 703-605-6000 (1-800-553-6847)

TDD 703-487-4639

Fax 703-605-6900

E-mail info@ntis.gov

Website http://classic.ntis.gov/

Reports are available to DOE employees, DOE contractors, Energy Technology Data Exchange representatives, and International Nuclear Information System representatives from the following source:

Office of Scientific and Technical Information

PO Box 62

Oak Ridge, TN 37831

Telephone 865-576-8401

Fax 865-576-5728

E-mail reports@osti.gov

Website http://www.osti.gov/contact.html

This report was prepared as an account of work sponsored by an agency of the United States Government. Neither the United States Government nor any agency thereof, nor any of their employees, makes any warranty, express or implied, or assumes any legal liability or responsibility for the accuracy, completeness, or usefulness of any information, apparatus, product, or process disclosed, or represents that its use would not infringe privately owned rights. Reference herein to any specific commercial product, process, or service by trade name, trademark, manufacturer, or otherwise, does not necessarily constitute or imply its endorsement, recommendation, or favoring by the United States Government or any agency thereof. The views and opinions of authors expressed herein do not necessarily state or reflect those of the United States Government or any agency thereof. 
Advanced Reactor Technology Program

Multiphysics simulations for molten salt reactor evaluation: Chemistry modeling and database development

\author{
J. McMurray ${ }^{1}$ \\ T. Besmann ${ }^{2}$ \\ J. Jerden ${ }^{3}$ \\ M. Williamson ${ }^{3}$ \\ J. Ard $^{2}$ \\ B. Fitzpatrick ${ }^{4}$ \\ M. Piro ${ }^{4}$ \\ A. Graham ${ }^{1}$ \\ Z. Taylor ${ }^{1}$ \\ B. Collins ${ }^{1}$ \\ B. Betzler ${ }^{1}$ \\ L. Qualls ${ }^{1}$ \\ T. Pandya ${ }^{1}$ \\ S. Greenwood ${ }^{1}$ \\ S. Johnson ${ }^{1}$
}

1. Oak Ridge National Laboratory

2. University of South Carolina

3. Argonne National Laboratory

4. University of Ontario Institute of Technology

Date Published:

June 2018

Prepared by

OAK RIDGE NATIONAL LABORATORY

Oak Ridge, TN 37831-6283

managed by

UT-BATTELLE, LLC

for the

US DEPARTMENT OF ENERGY

under contract DE-AC05-00OR22725 



\section{LIST OF FIGURES}

\section{CONTENTS}

LIST OF TABLES

1. INTRODUCTION

1.1 Modeling Framework Summary and Database Functional Requirements ..............................2

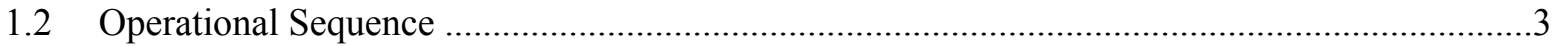

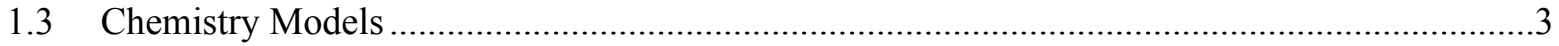

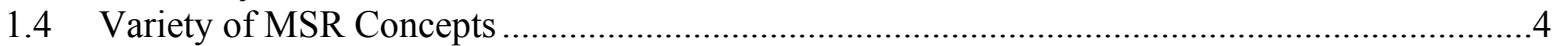

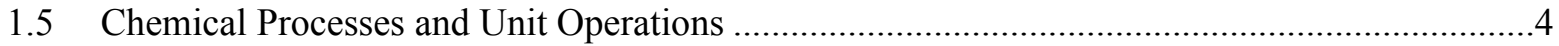

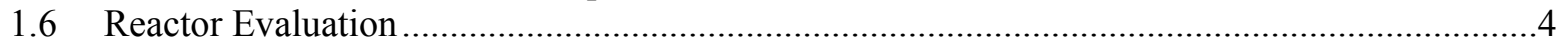

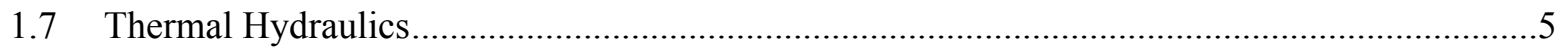

1.7.1 Implementation of General Species Transport in COBRA-TF ....................................

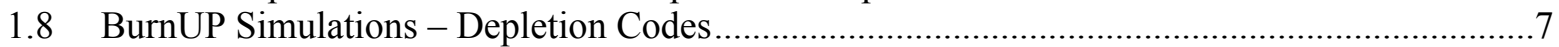

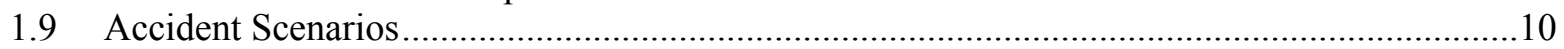

2. THERMOPHYSICAL DATA

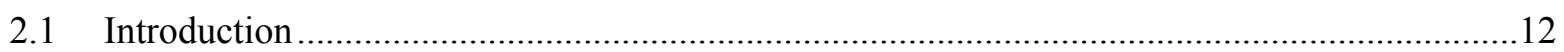

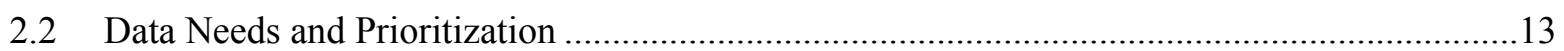

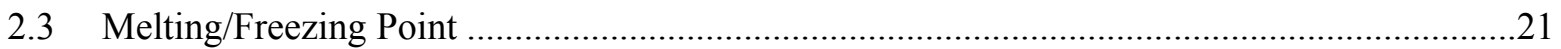

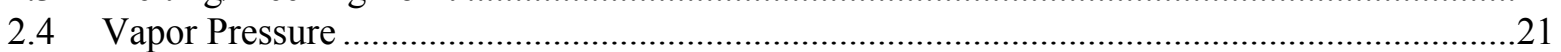

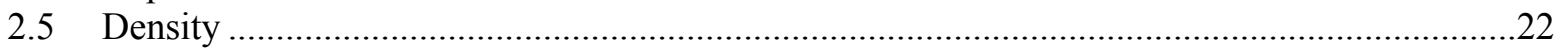

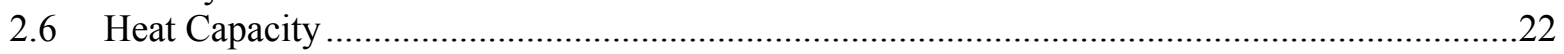

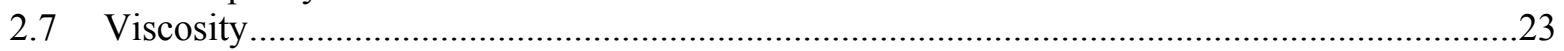

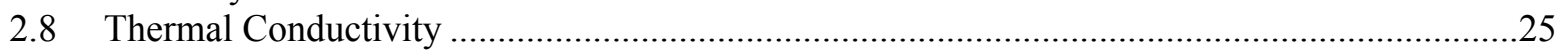

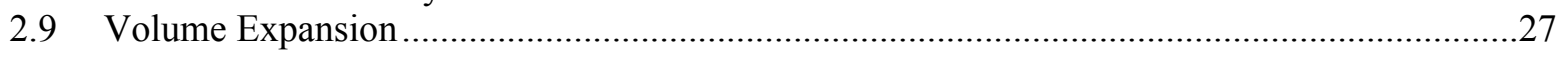

2.10 Dimensionless Coefficients for Fluid Dynamics/Heat Transfer Modeling ............................28

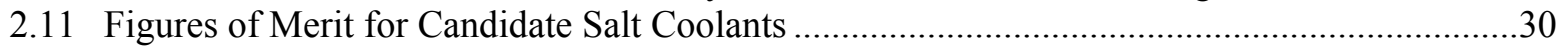

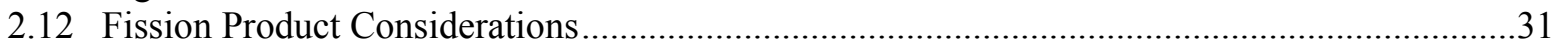

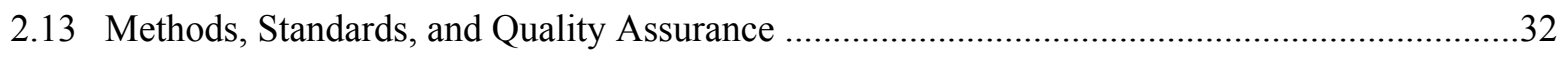

3. THERMOCHEMICAL MODELS AND DATABASE DEVELOPMENT

34

3.2 Applications

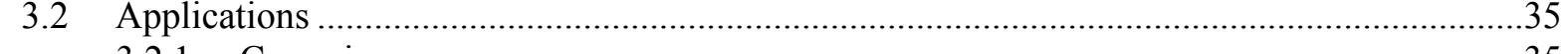

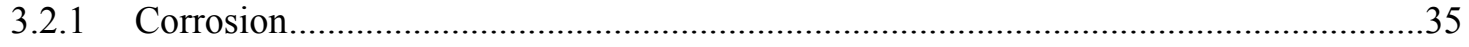

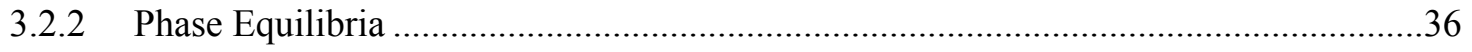

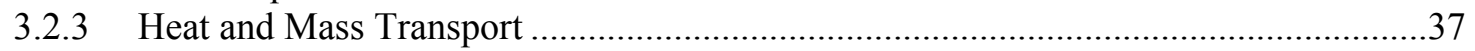

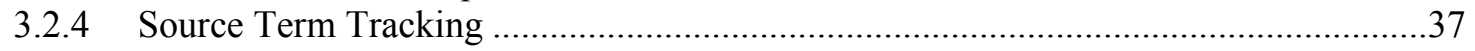

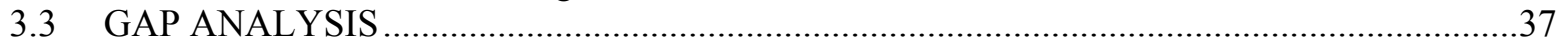

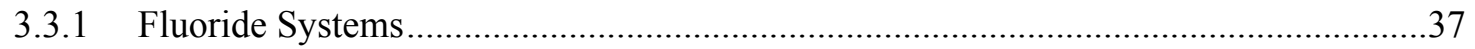

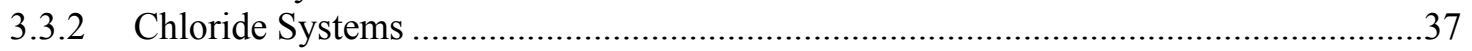

4. COUPLING CHEMISTRY TO REACTOR PERFORMANCE MOD-SIM

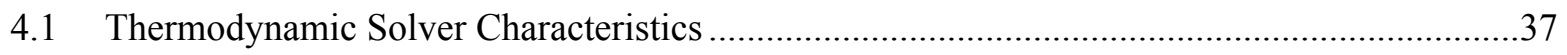

4.2 Thermochimica Development and Integration with ORIGEN and COBRA-TF ......................37

4.3 Thermophysical Data Integration into Mod-Sim Framework...................................................37 


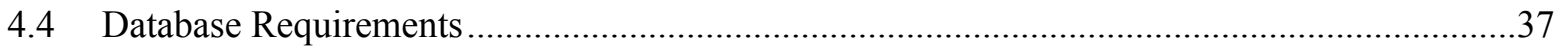

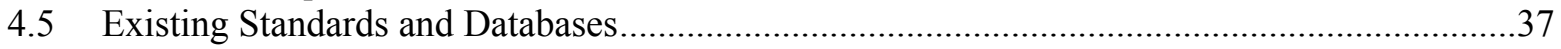

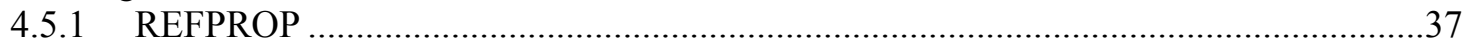

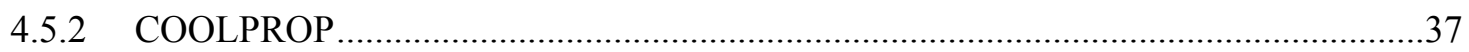

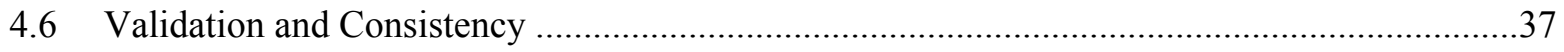

4.7 Functional Needs of a Thermophysical Molten Salt Database …............................................37

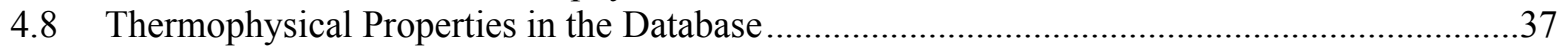

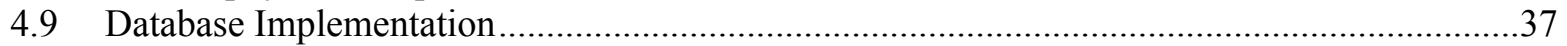

\section{FUTURE NEEDS}

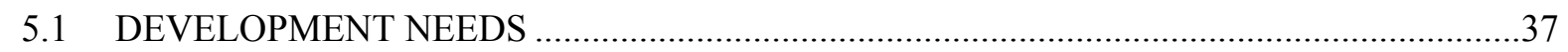

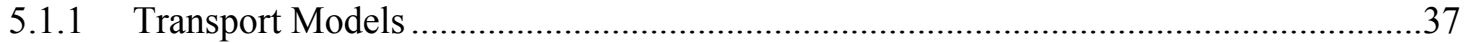

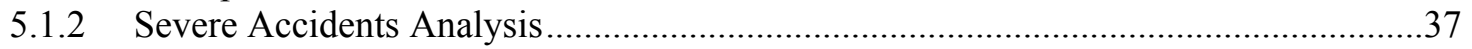

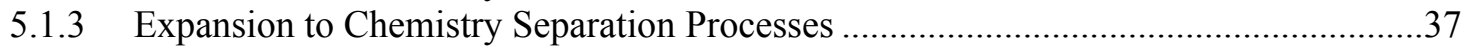

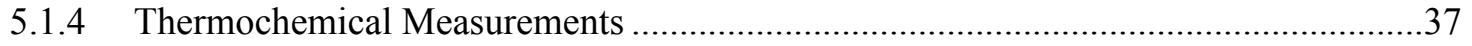

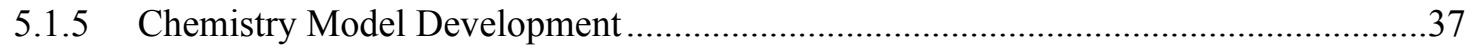

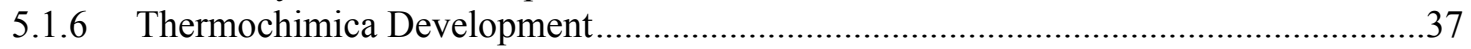

5.1.7 First Principles and Machine Learning Approach to Thermodynamic Values..............37

5.1.8 Thermophysical Property Models and Measurements..................................................37

ACKNOWLEDGEMENTS

\section{REFERENCES}

Appendix A. Demonstration of Implementation of Species Transport using COBRA-TF

Appendix B. Thermochemical Database Format

Appendix C. Example Thermochemical Database

appendix D: Thermochemical Models Code Value Key 


\section{LIST OF FIGURES}

Figure 1 MSR multi-physics simulation flow diagram illustrating the interconnectivity of individual phenomena......

Figure 2 Thermal hydraulic functionality inputs and outputs [1].

Figure 3 Fuel depletion functionality inputs and outputs [1]

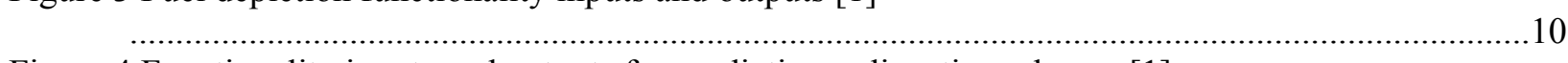

Figure 4 Functionality inputs and outputs for predicting radioactive releases [1].

Figure 5 Liquidous surface for the ternary salts $\mathrm{LiF}-\mathrm{NaF}-\mathrm{ZrF}_{4}$ and $\mathrm{NaCl}-\mathrm{KCl}-\mathrm{MgCl}_{2}$ showing temperature contours (light gray), and eutectic points (dark gray points) in ${ }^{\circ} \mathrm{C}$. The yellow region represents compositions that fall within the favorable melting point envelope, making them potential candidates as coolant salts.

Figure 6 Example of discrepancies between empirical fits to two different viscosity data sets for LiF-NaF-KF.

Figure 7 Example datasets showing viscosity as a function of temperature for selected salt compositions. (a) shows empirically derived functions from $[18,19]$ with $10 \%$ error envelopes, (b) shows measured data points from Cohen and Jones [49] and exponential trendlines with $5 \%$ error envelopes.

Figure 8 Example of density data from the literature for Li-NaF-KF highlighting discrepancies between measurements.

Figure 9 Computed fluorine activity versus redox (ratio of U(IV) to U(III)).

Figure 10 Liquidus projection and invariant points with phase boundaries for $\mathrm{LiF}-\mathrm{UF}_{4}-\mathrm{ThF}_{4}$ with fixed fractions of $0.05 \mathrm{CrF}_{2}$ and $0.05 \mathrm{UF}_{3}$. Computed using proprietary FactSage [77] database.

Figure 11 Liquidus projection and invariant points with phase boundaries for $\mathrm{LiF}-\mathrm{UF}_{4}-\mathrm{BeF}_{2}$ with fixed fraction of $0.05 \mathrm{UF}_{3}$. Computed using initial database from the JRC-Karlsruhe with added $\mathrm{UF}_{3}$ species.

Figure 12 Ruthenium species vapor pressures computed from equilibrium state of LWR fuel utilized in MELCOR.[79] ....

Figure 13 Cesium species vapor pressures computed from equilibrium state of LWR fuel utilized in MELCOR.[79] .....

Figure 14 Binary subsystems of LiF-BeF $-\mathrm{NaF}_{-} \mathrm{ThF}_{4}-\mathrm{PuF}_{3}-\mathrm{UF}_{4}-\mathrm{UF}_{3}-\mathrm{ZrF}_{4}-\mathrm{KF}-\mathrm{RbF}-\mathrm{CaF}_{2}$

Figure 15 Ternary subsystems of LiF-BeF $-\mathrm{NaF}_{2} \mathrm{ThF}_{4}-\mathrm{PuF}_{3}-\mathrm{UF}_{4}-\mathrm{UF}_{3}-\mathrm{ZrF}_{4}-\mathrm{KF}-\mathrm{RbF}-\mathrm{CaF}_{2}$

Figure 16 A schematic of input/output operations with Thermochimica.

Figure 17 Interfacing contribution of UOIT, between Thermochimica (thermodynamics),

ORIGEN (isotope physics) and COBRA-TF (thermal-hydraulics).....

Figure 18 Isotopic evolution of the noble metal system, from ORIGEN, using a power history from the MSRE. Power history taken from [100].

Figure 19 Thermodynamic simulation of phase evolution, from Thermochimica, coupled to the ORIGEN-API. Power history from [100].

Figure 20 Computed $\mathrm{K}-\mathrm{KCl}$ phase diagram using the solution model for the melt developed in this work with the thermodynamic values from the FactSage 7.1 database [77] for pure components and stoichiometric compounds. Symbols are experimental points from [101]. 
Figure 21 Predicted enthalpies (left) and entropies (right) of complex oxides via machine learning approach. About 100 oxides thermochemistry data obtained from experiments have been used to train linear regression model to predict formation enthalpies and Bayesian Ridge regression model to predict formation entropies at $25^{\circ} \mathrm{C}$, respectively.

Figure 22 Polyhedra-based features used to train machine learning models to predict thermochemistry of complex oxides. Examples shown here are perovskite and brownmillerite phases which have similarities in both stoichiometry and crystal structures. Polyhedra can simultaneously capture composition (with oxidation states of metal ions) and crystal structural information.

Figure 23 Diagram showing gaps addressed by R\&D activities to develop a state of the art thermophysical database/module for design and assessments of MSRs.

Figure 24 Qualitative assessment of the thermophysical properties database and predictive modeling methods for high temperature coolant salts by [19] (adapted).

Figure 25 Relative error numerical vs analytical solution for various time steps [2]

Figure 26 Relative error in ${ }^{135}$ I concentration for various axial meshes [2]

Figure 27 Step change in ${ }^{135}$ I concentration for various axial meshes [2]

Figure 28 Step change in ${ }^{135}$ I concentration for various lateral meshes [2]

\section{LIST OF TABLES}

Table 1 Examples of applications of molten salts in proposed and tested nuclear reactor concepts (adapted from [10]

Table 2 Thermophysical properties extracted from literature for MSR-relevant salts (adapted from [10]).

Table 3 Additional thermophysical properties extracted from literature for MSR-relevant salts (adapted from [10]).

Table 4 Fuel salt compositions studied by Capelli [41].

Table 5 Summary of theoretical and empirical predictive relationships for key salt properties. Each equation is discussed, and terms defined in more detail in the sections below.

Table 6 Comparison of measured heat capacities for candidate coolant salts with values calculated using the Dulong-Petit model (adapted from $[18,19]$ )

Table 7 Comparison of measured thermal conductivities with predicted values for candidate coolant salts (adapted from $[18,19]$ ).

Table 8 Comparison of volumetric expansion and other heat transfer properties for several relevant coolants.

Table 9 Key dimensionless coefficients for fluid dynamics.

Table 10 Convective heat transfer correlations for coolant flow within circular tubes (adapted from Samuel [10]based on equations from El-Wakil [60]and Incorpera, and Dewitt, D. P, 2002).

Table 11 Figures of Merit (FOM) equations used in the comparative analysis of molten salt coolant performance depending on flow regime (adapted from Samuel [10]). 
Table 12 Result from example calculations based on feature of merit equations defined in Table 2-11 above. Coolants are ordered based on values for forced turbulent convection pumping factor (FOM1).

Table 13 Main fluoride-based US MSR concepts [22, 80].

Table 14 Relevant MSR data and appropriate measurement technique. 


\begin{abstract}
To aid in design and licensing of molten salt reactors, a framework integrating the complex interaction of reactor neutronics, thermal hydraulics, and chemistry is being developed within the Department of Energy Advanced Reactor Technology Program's Molten Salt Reactor (MSR) campaign. The challenges of integrating thermochemical and thermophysical behavior into a multi-physics reactor simulations include the following: (1) population of data needed for refinement of current models and development of nonexistent models through experimental measurements, first principles calculations, and development of a machine learning approach (2) thermochemical and thermophysical model development, (3) further development of Thermochimica, an open-source efficient equilibrium solver used to link thermochemical models to the multi-physics code, (4) a framework for integrating kinetic phenomena: nucleation, precipitation, mass/heat transport, and corrosion models, and (5) a computational environment to efficiently utilize the data and models within a multi-physics modeling tool. These challenges are being addressed through a collaboration among Oak Ridge National Laboratory, Argonne National Laboratory, the University of South Carolina, and the University of Ontario Institute of Technology.
\end{abstract}

\title{
1. INTRODUCTION
}

This report relates to the purpose and the functionality of chemistry models used to simulate molten salt reactors and define the preliminary requirements for their implementation. The models will eventually span the range of salt chemistry needs from production through disposal, including accident and dose consequence evaluation. The chemistry models defined and described in this report are related to those models used to predict salt properties for multi-physics reactor evaluation for normal operation, including anticipated operational occurrences. Models related to chemistry for salt production, processing, or separation system description, and models related to severe accident analyses will be defined separately.

MSRs use high temperature, low pressure, salts as a reactor coolant, and in some cases, as the fuel. The behavior of the salt is integral to the behavior of the reactor and it must be well understood. For liquidfueled salts, fission occurs directly within the salt and fission products are born and begin to decay within the salt as they are dispersed through the primary system. Fission products, their decay daughters, in addition to transmutation and activation products make up the potential source term for an MSR. The behavior of these products is dependent upon the conditions within the reactor and specific composition of the salt being used, which is, by its nature, continuously changing. The chemistry challenges related to MSRs begins with salt production and purification and runs all the way though eventual salt waste disposal. A key factor in reactor licensing is related to radioactive material accountability, both during normal and accident scenarios, thus salt chemistry behavior must be understood over a broad range of salt conditions.

A fundamental difference between a liquid-fueled MSR and traditional reactors is the location of the radioactive source term under normal conditions. In liquid fueled reactors, the source term is dispersed within the salts, adhered to or absorbed in contacting materials, in effluent streams and processing systems, lost to the surrounding reactor containment areas, or lost to the environment. Tracking and accounting for the radioactive source term is a principal challenge for liquid-fueled MSRs and it is directly linked to understanding the chemistry underlying the salt behavior. Salt chemistry behavior is related to the following elements:

1) Thermo-physical properties of salts and their dependencies on operating conditions and compositional changes;

2) Thermochemical nature of salts, including their phases relationships, again, dependent on reactor operating conditions and salt composition; and 
3) Impact of salt composition and operating conditions on corrosion of primary structural materials.

The chemistry and corrosion models necessary to predict salt behavior within the reactor during normal operation are under development. The framework for these models is intended to be generic, so that any candidate reactor salt can be studied and modeled. This allows for parallel development of reactor concepts from multiple developers. As interest in a particular salt reactor increases, data for that salt can be collected and used. Where additional information is needed, experiments to generate new data can be commissioned. With appropriate data and models, important properties can be estimated through first principle predictions, and interpolation and extrapolation of measured data. With better data, salt properties can be estimated with better accuracy.

The MSR salt chemistry effort has the following basic elements: thermo-physical property data, thermochemical data and models that utilize them, and the computational framework to use the data within multiple models, and the database that stores data so that it can be efficiently accessed and used in those models. This document describes the chemistry data and models envisioned for modeling liquidfueled molten salt reactors and sets the preliminary requirements for the computational structure required to implement them. Information for salt-cooled, solid-fueled MSRs, and for salts used as heat transport fluids within secondary heat transport systems will be described by similar, but simpler, data sets and models.

\subsection{MODELING FRAMEWORK SUMMARY AND DATABASE FUNCTIONAL REQUIREMENTS}

The modeling framework consists of the following functionalities:

1) A depletion calculation based on reactor core conditions that calculates salt constituents based on operational history, including material additions and removal from the salts

2) A multi-physics modeling framework that calculates neutronics, thermal-hydraulics, and mass transport within the system based on salt chemistry

3) A thermodynamic chemistry model

4) A reactor transient system model with transport models

Evaluation methods are under development that can perform integrated MSR analysis. These programs are examples of a broader set of tools that can perform similar analyses. The chemistry models and databases developed should be broadly applicable to modeling platforms. The depletion tool used is ORIGEN. The multi-physics code under development is VERA-MSR, which is derived from the VERA code used to model chemistry and material deposition within light water reactors. Thermochimica is an open source thermodynamic model developed to work with high performance computing codes. TRANSFORM is a reactor system dynamic modeling program developed in the open-source Modelica programming environment.

Depletion analysis is used to predict salt isotopic concentrations based on original salt composition, reactor core design, operation, and salt processing history. The isotopic concentrations produced are compressed into elemental molar concentrations and input into a modeling tool that uses the CALPHAD thermodynamic method in combination with collected data and modeling to develop salt structure, phase information, and other thermodynamic functions of the specified compositions. Thermochimica is the computationally-efficient open-source implementation of the CALPHAD method that is used to produce these thermodynamic functions. It is designed for integration with High Performance Computing (HPC) multi-physics modeling tools. Because it is an open source software package, it can be broadly shared and used within the MSR community. Thermochimica takes molar elemental combinations as input and calculates the lowest energy state, from which the equilibrium chemical combinations can be predicted. 
For liquid salts in an MSR, the assumption that the mixtures achieve equilibrium instantaneously is an acceptable approximation.

The data that Thermochimica relies on is derived from available experimental, salt chemistry data. Data for specific salt combinations are collected and stored in specific file formats. The files are created manually and stored in various databases controlled by users in the scientific community. Specific salt chemistry data files can be shared under cooperative agreements or new data files can be manually created from data found through literature searches or through experiments. Thermochimica can calculate specific heat of a salt. However, it cannot produce estimates of density, viscosity, or thermal conductivity. These thermo-physical properties, important for reactor performance evaluation, must be generated through experimental measurement. They will also be stored within a related database.

Normal operation is unique to a specific reactor design, as are operational upsets that can occur. For MSRs, normal operation is dependent on the salt used, the specifics of the reactor design, and the processes used to modify the salts to maintain the desired operational states. The radioactive source term for MSRs may be located in multiple systems around the plant. Each of these systems and locations require evaluation. Salt chemistry models are used to predict the behavior of specific salt compositions under anticipated operating conditions. These compositions vary over time, and the models must be able to estimate performance over the ranges of interest, even when there is a lack of experimental data.

\subsection{OPERATIONAL SEQUENCE}

For an analysis, the following sequence is envisioned:

1) The user specifies initial salt composition or reads a composition as an input file from a previous evaluation.

2) The operational history proposed is used within the depletion evaluation to estimate salt compositions as a function of time.

3) The thermophysical properties are estimated for the salt compositions based on available data.

4) Thermochimica is executed to determine expected thermodynamic properties of the salt

5) A multi-physics evaluation of the reactor system is operated that uses neutronics, thermalhydraulic, thermochemistry, and material transport models, to predict the distribution of the source term within the system.

6) The results of the multi-physics evaluation are input into a reactor system model, which uses emulation transport models to predict reactor system behavior during transient scenarios.

Preliminary implementation of the models assumes that changes in the salt composition do not impact thermophysical or thermochemical property values between evaluation time steps. However, this assumption requires examination to properly set the time steps. MSRs are low-pressure machines. The inputs to the database will not include pressure and will initially include only temperature and salt composition.

\subsection{CHEMISTRY MODELS}

In a liquid-fueled MSR, the thermophysical properties of the primary salt influence reactor performance. Density, viscosity, thermal conductivity, optical transmission, and heat capacity, among others, must be known to properly design the reactor and maintained to properly operate and control the reactor. For liquid-fueled MSRs, the unstable isotopes within the salt influence reactor power in a manner that is unique compared to traditional solid fuel reactor systems. Delayed neutrons can be emitted outside of the reactor core in non-critical areas and can cause fissions but not contribute to the core chain reaction. 
Other neutron emitters may pass around the primary system before returning to the core to release their neutrons. Thus, the flow rate of the salts, the distances they travel, and the time to neutron emission are intimately connected to reactor behavior. As salts pass around the primary system, their temperatures, pressures, flow rates, and irradiation fields all change. The composition of the salt evolves spatially and temporally. These changes must be understood and their importance to the key questions of reactor behavior and source term behavior and accountability must be considered.

Salt composition impacts reactor performance behavior and it directly influences interaction with contacting structural materials. To prevent excessive corrosion, the salt properties must be maintained within acceptable bounds. Compositional changes from fission and transmutation products, and, perhaps to a lesser extent, from corrosion of structural materials, inevitably affect the equilibrium state. Thus, the significant features of the salt (those things that matter), have to be known, monitored and controlled. The ability to predict and monitor them and the means of manipulating them require development. Thus, salt chemistry models and salt monitoring instrumentation require development.

\subsection{VARIETY OF MSR CONCEPTS}

Multiple MSR reactor concepts are under development. Each developer is evaluating materials and salt combinations in an effort to make viable concepts for the market. It is not possible at this time to identify and concentrate on a particular MSR technology. Thus, early efforts related to chemistry modeling development are focused on developing generic frameworks that can address a broad range of salt chemistry and materials compatibility issues. As concepts mature, specific data from specific salts will be used within these frameworks to evaluate salt chemistry within the context of a specific reactor design.

\subsection{CHEMICAL PROCESSES AND UNIT OPERATIONS}

Within a liquid-fueled MSR, the salt is constantly changing. It is modified through fuel additions, intentional separations processes, and unintentional losses. Separation processes are the real systems that implement the chemistry used for extraction and separations. These processes are imperfect and may contaminate the salt with trace amounts of impurities. All constituents within the salts potentially have significant impact on reactor performance, plant maintenance, salt processing, storage systems, or final waste disposition.

\subsection{REACTOR EVALUATION}

The complex interaction of reactor neutronics, thermal hydraulics, and dynamic salt chemistry within a liquid fueled MSR requires an integrated evaluation methodology. For most reactors types, neutronics and thermal hydraulics can be performed separately and iteratively, and coolant chemistry can be entirely isolated. For liquid-fueled MSRs, they must be performed together.

The models that are to be used to describe the salt composition, reactor conditions, and salt processing system performance require definition, development, and implementation in such a manner that they can be efficiently used in reactor analysis. Models can consist of the following:

1) Analytical equations

2) Empirical relationships derived from data

3) Fits to mathematical forms based on a combination of theory, experience, and data

4) Combination of all of the above. 
The data that populate models is specific to salt compositions, of which there are many to consider, and liquid-fueled salts will vary continuously in composition and operate over a large range of conditions. Data is needed over the possible conditions encountered. However, there must be methods to estimate properties where data does not exist and interpolate and extrapolate among both conditions and contents simultaneously. The chemistry models represent a utilitarian embodiment of the data used to develop them. They facilitate creation of a framework for efficient and effective means of accessing and using that data. The need to interpolate between measured data points and to extrapolate beyond them, both in terms of composition, structure, and condition is also required. Figure 1 shows a representation of how chemistry data, chemistry models are used within a multi-physics reactor evaluation.

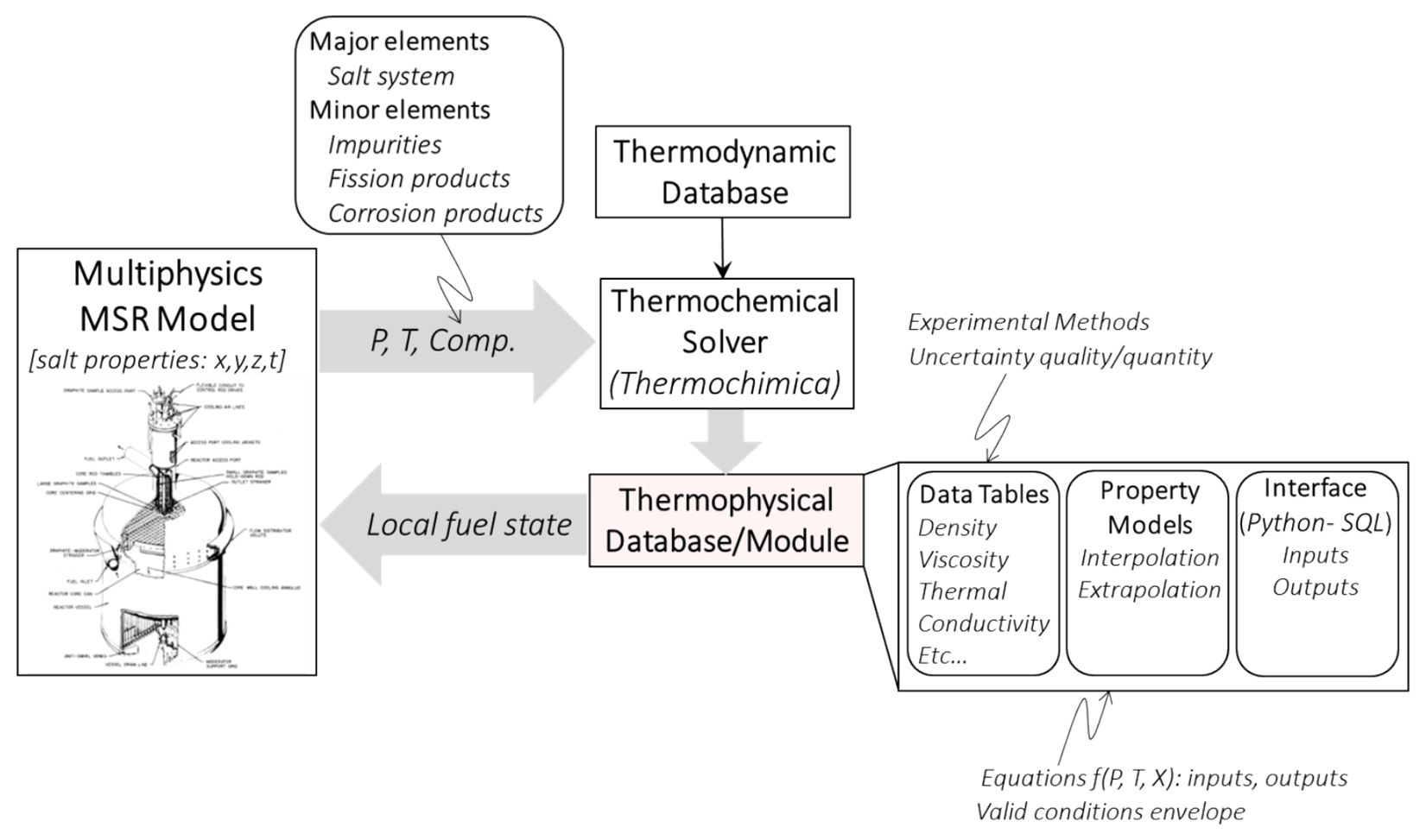

Figure 1 MSR multi-physics simulation flow diagram illustrating the interconnectivity of individual phenomena.

\subsection{THERMAL HYDRAULICS}

Thermal hydraulics is a critical piece in characterizing reactor performance and safety, calculating heat transfer and fluid flow and generating temperatures, pressures, and flow rates. For solid-fueled systems, the majority of the heat is generated within the fuel material; heat is transferred from the fuel to the coolant through cladding and other fission product barriers. The effectiveness of this heat transfer is imperative for reactor safety, as peak temperatures exist with the fuel material, which have specific limits to prevent fuel failure. Traditional thermal hydraulic tools are specialized for simpler cylinder-channel light water reactor geometries, which provides for simplified heat transfer and fluid flow physics. Some developments for pebble bed thermal hydraulics were made for gas-cooled high-temperature reactors; additional considerations are required for fluoride salt-cooled systems due to the differences between gaseous and salt coolants. 
In liquid-fueled molten salt reactors, the majority of heat is generated within the fuel salt, which in most cases doubles as the coolant. The fuel salt heats adjacent moderator and structural components; material (e.g., temperature) limits for these components exist. Thermal and fast spectrum liquid-fueled molten salt reactor systems present very different thermal hydraulics problems; most fast spectrum systems consist of an open core and thermal spectrum systems contain moderator structure with cylindrical fuel salt channels. For open core systems and component design and development, high-fidelity computational fluid dynamics may be required.

Coupling between core neutronics, thermal hydraulics, and chemistry is required to accurately characterize the steady-state (or time-dependent for transients) performance of the reactor (e.g., flux, temperature, flow). Temperature-dependent core neutronics simulations provide thermal hydraulics tools with power distributions. For salt reactors, the accuracy of these thermal hydraulic simulations is largely dependent on the salt thermophysical properties (e.g., viscosities, thermal conductivities, heat capacity, thermal diffusivity) for a range of conditions (e.g., temperatures, pressures, salt impurities). Chemistry models provide feedback to these calculations.

In summary, nuclear thermal hydraulics tools rely on accurate thermophysical data for the coolant or fuel salt (Figure 2). These tools provide spatially-resolved temperatures, flow rates, and pressures that feed chemistry models to obtain localized behavior of salt constituents (e.g., fission products in heat exchangers or noble metals at structural interfaces).

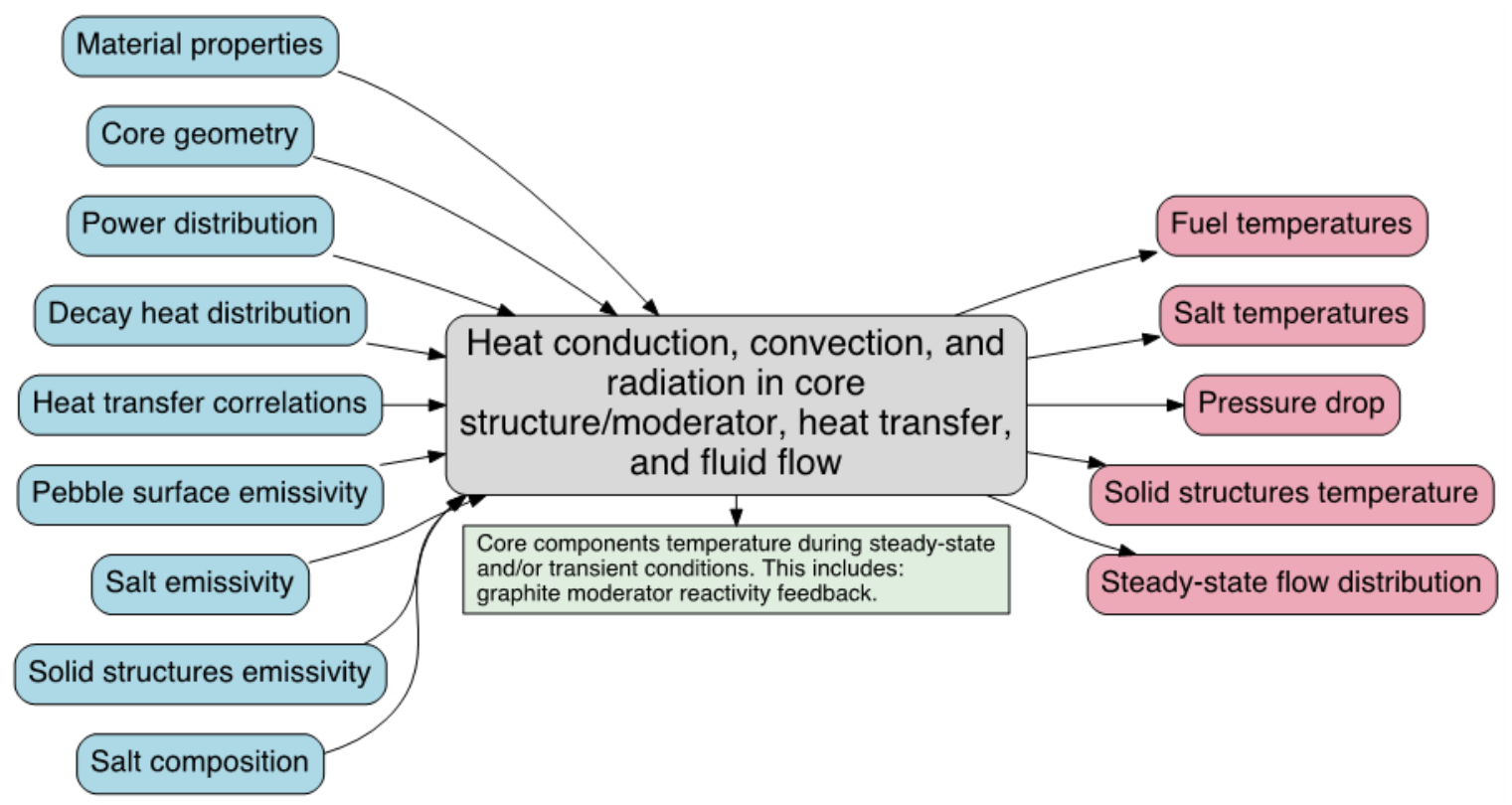

Figure 2 Thermal hydraulic functionality inputs and outputs [1].

Implementation of species transport yields elemental concentration information for predicting the chemical state. Once the chemical state is calculated and known, this is feed back into species transport. 
Heat evaluation and transport also feeds into calculating the thermophysical state. Understanding and modeling heat transport yields temperature and pressure distribution which are both inputs in determining the thermophysical state.

\subsubsection{Implementation of General Species Transport in COBRA-TF}

CTF is a modernized version of COBRA-TF a thermal hydraulics (TH) code currently being utilized as the main TH solver for the VERA-CS modeling and simulation package. CTF uses a two-fluid modeling approach with flow divided into three field: fluid film, vapor and liquid droplets [2]. Governing transport equations for a single phase are discretized on a finite volume represented below.

$$
\frac{\partial}{\partial t} \iiint_{V} C_{j} d V=-\iint_{S} n \cdot F_{j} d S+\iiint_{V} B_{V, j} d V+\iint_{S} B_{S, j} d S
$$

The term on the left-hand side represents the change in concentration of species $\mathrm{j}$ with respect to time. Continuing to the right, the first term on the right-hand side is the flux of species $\mathrm{j}$ into/out of the current mesh cell. This flux will have contributions from convection and or diffusion terms. Next is the volumetric source term followed by the surface source term for species $\mathrm{j}$. The first term can be simplified using the Leibniz rule and noting that the control volumes are fixed. The terms $C_{j}, B_{v, j}$, and $B_{s, j}$ can be represented by their volume averaged terms.

$$
\bar{C}_{j}=\frac{1}{V} \iiint_{V} C_{j} d V \quad \bar{B}_{v, j}=\frac{1}{V} \iiint_{V} B_{v, j} d V \quad \bar{B}_{s, j}=\frac{1}{A} \iint_{A} B_{s, j} d A
$$

After application of these volume average terms and some algebraic manipulation, the single-phase transport equations become;

$$
\frac{\partial \bar{C}_{j}}{\partial t}=-\frac{1}{V} \iint_{S} n \cdot F_{j} d S+\bar{B}_{v, j}+\frac{A}{V} \bar{B}_{s}
$$

The flux term is modeled by ignoring the diffusive term and applying an upwind difference scheme for the convective term. Convection is then represented by a double summation. First is a summation over each face of the cell, next is a summation over the connected cells to that face. Solving this system is accomplished by applying an explicit numerical scheme for the time dependent term, allowing for a direct and simple solution.

\subsection{BURNUP SIMULATIONS - DEPLETION CODES}

Burnup or depletion simulations are used in nuclear engineering to calculate the change in a material isotopic composition over time due to continuous neutron irradiation. Mathematically, this involves solving a large set of Bateman equations describing the decay and irradiation of nuclides, where the rate of change of nuclide describe the rate of change of the nuclides in the problem, where the rate of change of nuclide $i$ is

$$
\frac{d N_{i}}{d t}=\sum_{j=1}^{m} l_{i j} \lambda_{j} N_{j}+\bar{\Phi} \sum_{k=1}^{m} f_{i k} \sigma_{k} N_{k}-\left(\lambda_{i}+\bar{\Phi} \sigma_{i}\right) N_{i}
$$

where $N_{i}, N_{j}$, and $N_{k}$, are the number densities of nuclides $i, j$, and $k$;

$l_{i j}$ is the branching fractions of radioactive decay from nuclide $j$;

$\lambda_{i}$ and $\lambda_{j}$ are the decay constants of nuclides $i$ and $j$; 
$\bar{\Phi}$ is the space- and energy-averaged neutron flux;

$f_{i k}$ is the branching fraction for neutron absorption by other nuclides $k$ that lead to the formation of nuclide $i$; and

$\sigma_{i}$ and $\sigma_{k}$ are the spectrum-averaged neutron absorption cross sections of nuclides $i$ and $k$.

The three terms on the right-hand side of the equation represent (1) the decay rate of nuclide $i$ into nuclide $j$, (2) the production rate of nuclide $i$ from irradiation, and (3) the loss rate of nuclide $i$ due to decay and irradiation. Thus, this includes production and destruction from fission, transmutation, and decay. Generally, the flux and cross sections are generated during a neutron transport calculation, as the remaining elements in these equations are nuclear data. In all nuclear engineering calculations, elements are tracked by individual isotope, as nuclear data may vary widely for isotopes of the same element. Chemical compound forms are of low or no importance in these simulations. In SCALE [3], the ORIGEN tool [4] solves this set of Bateman equations.

The evolution of a material composition has a large impact on criticality, radioactivity, decay heat, and chemical composition. In most solid-fueled reactors, fission products and actinides remain physically with the initial fuel material during and after operation until reprocessing (or indefinitely in a once-through fuel cycle). This is due to fission product barriers (e.g., fuel rod cladding and TRISO particle structure) that are designed for containment. As bound uranium atoms undergo fission, resulting fission products form chemical compounds that have an impact on the solid structure of the fuel and pellet-clad interactions. Non-reactive fission product gases may migrate within the solid fuel structure. These concerns are relevant for solid fueled fluoride salt-cooled high temperature reactor systems, which uses a TRISO particle-based fuel form.

A liquid-fueled reactor is likely to have online separations and/or feeds, where material is moved to or from the core at all times (continuous) or at specific intervals (batch). The ability to perform online separations improves the potential neutronic performance of liquid-fueled systems. For example, it is unnecessary for liquid-fueled systems to operate with excess reactivity if fissile material is continuously being fed into the core. There is also an additional neutronic benefit from removing fission products with high concentrations and absorption cross sections, but removal of each element from the liquid fuel presents a unique issue in terms of separations, storage, and disposal of the radioactive materials.

To account for batch discards, a depletion tool must have the ability to remove a fraction or total amount of a material at a specified time. Accounting for a continuous removal or addition is more difficult because it requires adding a term to the Bateman equations,

$$
\frac{d N_{i}}{d t}=\sum_{j=1}^{m} l_{i j} \lambda_{j} N_{j}+\bar{\Phi} \sum_{k=1}^{m} f_{i k} \sigma_{k} N_{k}-\left(\lambda_{i}+\bar{\Phi} \sigma_{i}-r_{i}\right) N_{i},
$$

where $r_{i}$ is the external loss constant for nuclide $i$. For a solid-fueled reactor, $r_{i}$ is zero, and in many depletion tools this is a hard-coded assumption. For example, the SCALE/TRITON tool [5] does not allow for the specification of non-zero removal or feed rates for depletion simulations, though ORIGEN input allows the specification of these rates. Due to the Bateman equation form, these rates must be expressed in terms of a decay constant. An accurate removal/feed rate must take into account processing system design and efficiencies, liquid fuel flow rates, and reactor design. For example, fission product gasses that are continuously generated during operation may form chemical compounds, be removed actively in a processing system, or removed passively during interaction with the cover gas. Thus, chemical behavior, volatility, and solubility are all important for quantifying the removal rate of a given element. From a depletion tool perspective, all of this information is rolled into the single constant $r_{i}$, as only the isotopic composition is important. An infinitely mixed assumption simplifies the depletion simulations, as this treats the fuel salt as a single material. 
Some tools have been developed to solve the Bateman equations with the additional feed and removal terms. One reactor physics-based approach uses a semi-continuous batch process to simulate a continuous process to model the changing isotopic composition of the liquid fuel in a molten salt reactor during operation. The ChemTriton tool models salt treatment, separations, discards, and fueling using an MSR unit cell model, iteratively running SCALE/TRITON over small time steps to simulate continuous processes and deplete the fuel salt [6]. This tool has been used to generate fuel cycle behavior for the Fuel Cycle Options Campaign. Additional developments in SCALE are ongoing to deliver higher accuracy results [7]. Regardless, the ChemTriton tool is able to generate the isotopic composition over the lifetime of a reactor using assumptions in fissile/fertile material feed rate and isotopic removal rates. These are easily collapsed into elemental compositions, as the isotopic detail is less important for salt chemistry.

In a liquid-fueled system, the fuel salt is continuously changing in time. But, the rate of this change and its effect on the change in chemical behavior of the fuel salt has not been well-characterized and is design dependent. Systems with larger amounts of fuel salt, a low power density, and fission product processing systems may undergo a relatively gradual change in isotopic composition with respect to integral systems with a high-power density and no fission product removal systems. From a reactor design perspective, it is illogical to design a system that has the potential for a rapid change in chemical behavior, for which control or mitigation systems would not be able to correct.

In summary, depletion simulations mostly feed the chemistry models that depend on elemental composition (Figure 3). But, for liquid-fueled systems, some feedback is necessary to better quantify (1) elemental removal rates and (2) the necessary feed rate of chemistry control or corrosion mitigation materials. 


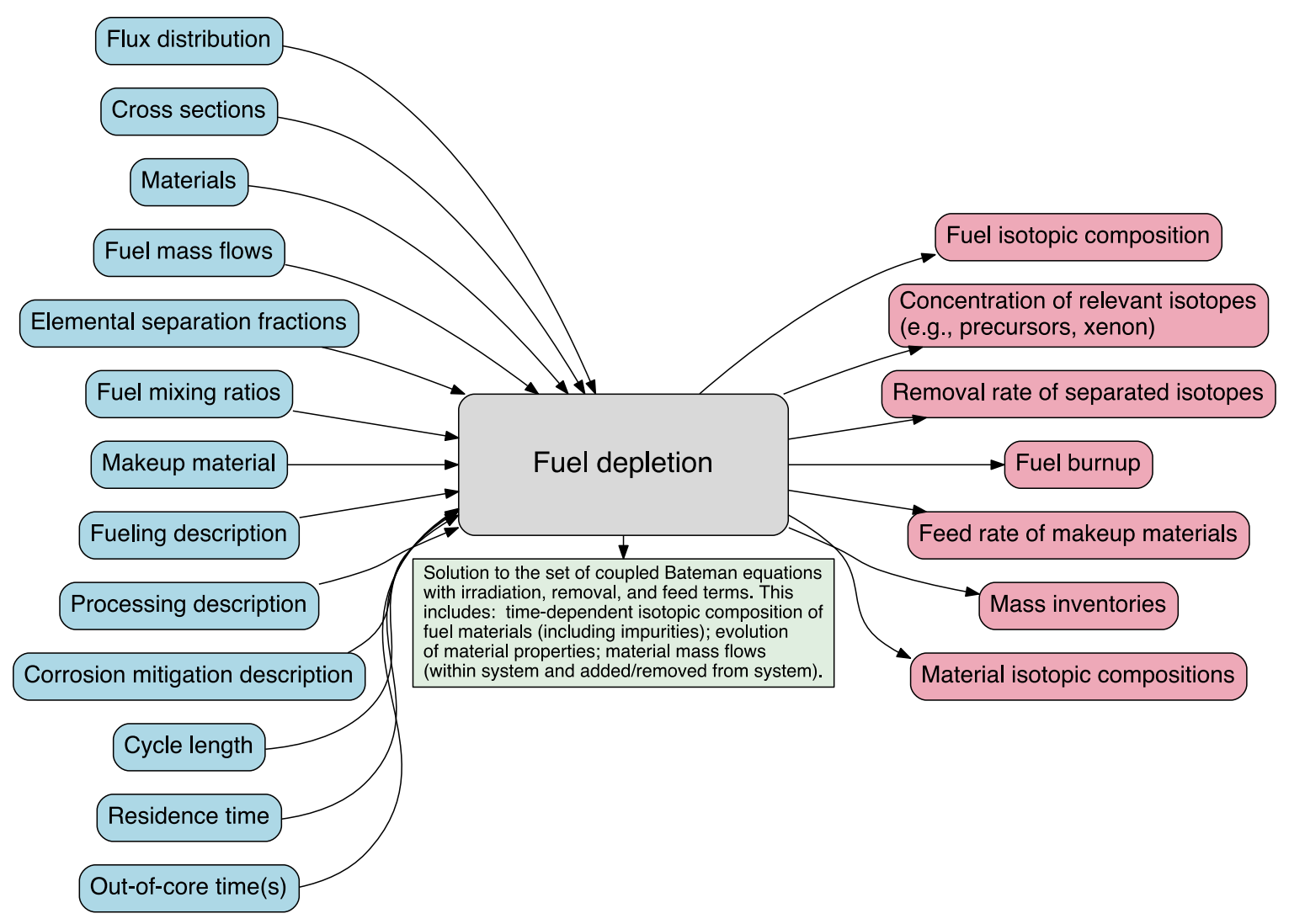

Figure 3 Fuel depletion functionality inputs and outputs [1]

\subsection{ACCIDENT SCENARIOS}

Accident analyses in a reactor licensing case include both design basis accidents and severe (i.e., beyond design-basis) accidents. It must be shown that a reactor responds safely to design basis accidents and does not exceed radiological release limits. A severe accident analysis provides radiological release information for siting and early release limits. These determinations are made from a set of calculations based on the nuclear and thermal hydraulic design, fuel, materials, structure, instrumentation, control systems, and a set of accident sequences and initiators [8]. While some accident sequences for molten salt reactors are known, many are design dependent and have yet to be defined.

In solid-fueled reactors, several physical barriers exist between the fuel material and a potential radiological release in a designed defense-in-depth approach [9]. Multiple physical failures are required to result in a radiological release. In a light water reactor, radioactive fission products must escape the fuel pellet, travel through the cladding, cross the coolant boundary, and breach reactor containment before a radiological release to the public. For solid-fueled fluoride salt-cooled high temperature reactors using a TRISO fuel form, an additional defense-in-depth layer is added in the form of a contained microsphere particle. The progression to radiological release in these systems is very similar to a pebble bed gascooled high-temperature reactor, with the main difference being the gaseous coolant instead of the fluoride salt coolant. Contributors to the failure of these physical components are peak temperatures and energy density. 
In liquid-fueled molten salt reactors, the fuel material may no longer be encased in a solid cladding and may be free to flow from the core region to heat exchangers and processing systems. A defense-in-depth approach still exists: systems will still have a fuel salt boundary, a coolant salt boundary, and reactor containment. One barrier to radiological release in these systems may be the liquid salt itself, in which certain chemical compounds (fuel, fission products, etc.) will remain in solution under accident conditions. For example, some volatile compounds will escape the salt to the cover gas during normal operation, but this may change during a transient in which the salt temperature fluctuates. In addition, the source term of the fuel salt may be reduced in systems with active fission product removals, as the most volatile components are likely being separated from the fuel materials.

From an operations and design perspective, it is important to understand the effects of fluctuations in power density and temperature within the core and primary coolant loop. During normal operation, there should be some level of understanding of salt constituent (e.g., noble metals and tritium) behavior, from their creation to removal, transport, or deposition onto a surface. During an accident scenario, both the source rate of salt constituents and salt and material (e.g., graphite and alloys) temperatures may fluctuate. Thus, salt constituents that have deposited onto a surface may reenter the salt with an increase in material temperature. It is important to understand these behaviors to understand the source term within the salt and the condition of components (e.g., heat exchangers and pipes).

For light water reactors, MELCOR and MACCS are the severe accident analysis tools for integrated analysis and atmospheric release, respectively. Depletion simulations generate fuel material compositions (e.g., heavy metals, fission products) that are fed to initiate the accident progression sequence. These tools simulate the accident progression to eventual radiological release.

The ability of a liquid salt to act as a radiological release barrier (i.e., retain constituents within the salt) is inherently a chemistry-dependent characteristic (e.g., solubilities and volatilities of salt constituents). For any practical application, this ability must be understood across a variety of conditions that the salt may encounter during a transient scenario (e.g., temperatures, concentrations, exposure). Transient scenario timescales may range between milliseconds to minutes or longer; the chemistry models must be appropriate (e.g., reaction kinetics) to capture rapid changes in the salt condition.

In summary, the nuclear and thermal hydraulic progression (e.g., peak temperatures, energy deposited) of accident scenarios are mostly understood without chemistry models, instead relying on reactor physics, kinetics, heat transfer, and salt thermophysical properties. But, the consequences of the accident progression on potential radiological releases is inherently dependent on chemistry models (Figure 4). 


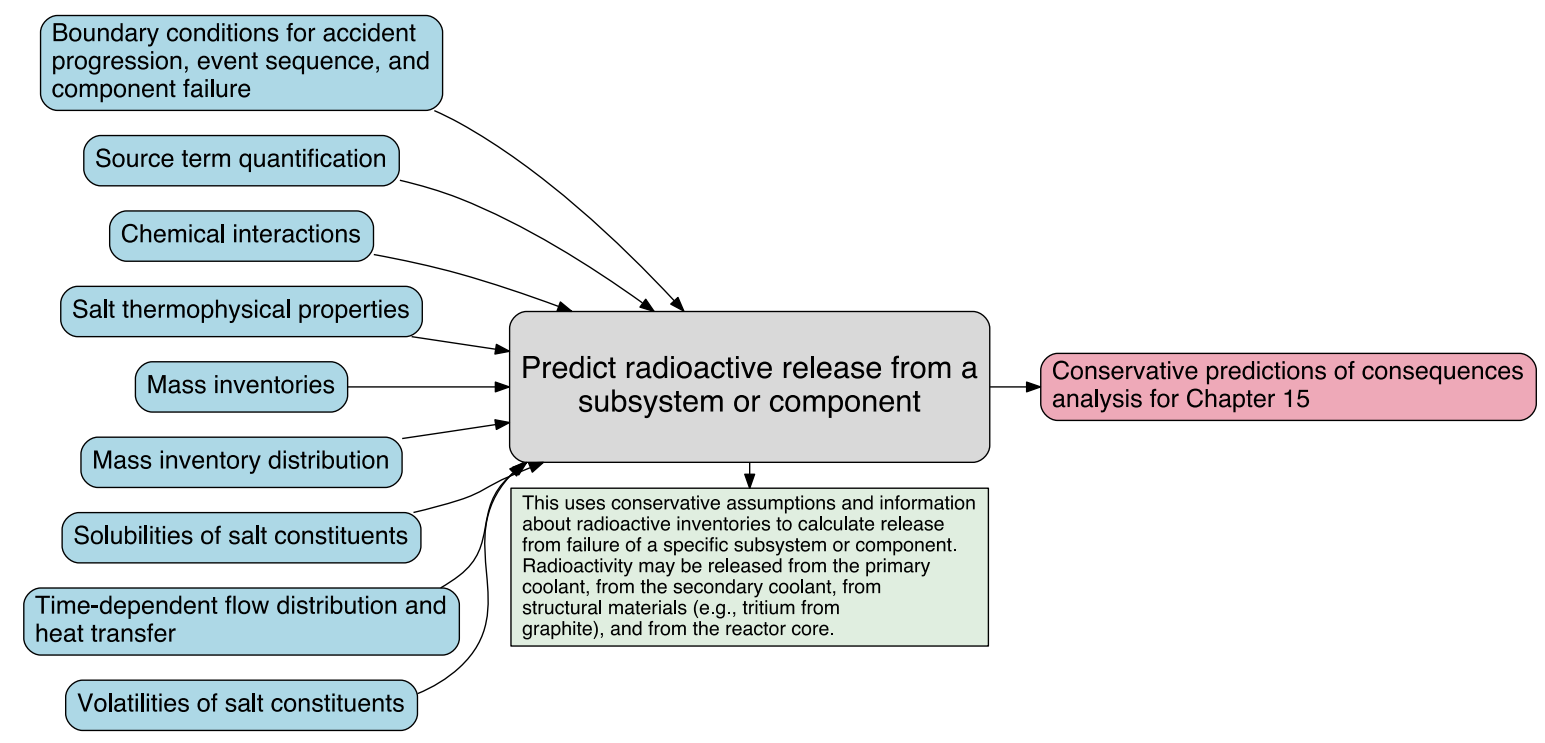

Figure 4 Functionality inputs and outputs for predicting radioactive releases [1].

\section{THERMOPHYSICAL DATA}

\subsection{INTRODUCTION}

Table 1 lists examples of molten salt compositions proposed for applications in Generation IV nuclear reactor technologies. As indicated in Table 1, there are three general applications of molten salts in these advanced nuclear reactor systems:

- Salts used as a fuel and coolant that dissolve fissile $\left({ }^{235} \mathrm{U},{ }^{233} \mathrm{U},{ }^{239} \mathrm{Pu}\right)$ and/or fertile $\left({ }^{232} \mathrm{Th},{ }^{238} \mathrm{U}\right)$ actinides. This application is used in MSR breeder and burner technologies.

- Salts used as a primary coolant only that are circulated in the primary cooling loop.

- Salts used as heat exchange media in secondary loops and heat exchangers.

The fuel and coolant salts consist of binary, ternary or quaternary salt mixtures that contain the fissile actinides that fission in the reactor core and are circulated within the primary loop to the primary heat exchangers during reactor operation (e.g., reference [10]). Heat from the primary heat exchangers may then be transferred to a secondary coolant salt that does not contain fissile or fertile elements. In saltcooled solid fueled reactors (e.g., liquid salt cooled reactors), the molten salt is restricted to circulation within the primary cooling loop to transport heat from the core to intermediate loops that may also use liquid salts to transport heat to either a Brayton power cycle for electricity production or to a hydrogen production facility $[10,11]$. 
Table 1 Examples of applications of molten salts in proposed and tested nuclear reactor concepts (adapted from [10]

\begin{tabular}{|c|c|c|c|c|}
\hline Reactor Type & $\begin{array}{l}\text { Neutron } \\
\text { Spectrum }\end{array}$ & $\begin{array}{l}\text { Molten Salt } \\
\text { Application } \\
\end{array}$ & Reference Salt Systems & Selected References \\
\hline \multirow{5}{*}{$\begin{array}{l}\text { Molten Salt } \\
\text { Breeder Reactor }\end{array}$} & Thermal & Fuel & ${ }^{7} \mathrm{LiF}_{-} \mathrm{BeF}_{2}-\mathrm{AnF}_{4}$ & {$[12-14]$} \\
\hline & \multirow{4}{*}{ Fast } & $\begin{array}{l}\text { Secondary } \\
\text { coolant }\end{array}$ & $\mathrm{NaF} \mathrm{NaBF}_{4}$ & {$[12-14]$} \\
\hline & & \multirow{3}{*}{ Fuel } & ${ }^{7} \mathrm{LiF}-\mathrm{AnF}_{4}$ & {$[12-14]$} \\
\hline & & & $\begin{array}{l}\mathrm{NaCl}-\mathrm{MgCl}_{2}-\mathrm{UCl}_{3}- \\
\mathrm{PuCl}_{3}\end{array}$ & {$[15]$} \\
\hline & & & $\mathrm{LiF}-\mathrm{NaF}-\mathrm{BeF}_{2}-\mathrm{AnF}_{3}$ & {$[16]$} \\
\hline $\begin{array}{l}\text { Advanced High } \\
\text { Temperature } \\
\text { Reactor }\end{array}$ & Thermal & $\begin{array}{l}\text { Primary } \\
\text { coolant }\end{array}$ & ${ }^{7} \mathrm{LiF}-\mathrm{BeF}_{2}$ & {$[17-19]$} \\
\hline $\begin{array}{l}\text { Very High } \\
\text { Temperature } \\
\text { Reactor }\end{array}$ & Thermal & $\begin{array}{l}\text { Heat } \\
\text { transfer } \\
\text { coolant }\end{array}$ & LiF-NaF-KF & {$[18,19]$} \\
\hline \multirow{2}{*}{$\begin{array}{l}\text { Liquid Salt } \\
\text { Cooled Fast } \\
\text { Reactor }\end{array}$} & \multirow{2}{*}{ Fast } & $\begin{array}{l}\text { Primary } \\
\text { coolant }\end{array}$ & LiCl-NaCl-MgCl 2 & {$[11,20]$} \\
\hline & & $\begin{array}{l}\text { Intermediate } \\
\text { coolant }\end{array}$ & $\mathrm{NaNO}_{3}-\mathrm{KNO}_{3}$ & {$[21]$} \\
\hline
\end{tabular}

The specific salt systems and compositions that are best suited for particular applications will be based on their thermophysical and chemical properties. Several thermodynamic and physical properties databases for molten salts have been assembled and several review papers and reports summarize the substantial literature in this field $[10,18,19,22,23]$. However, there remains a need for a functional database and computational tool that can be used to select, optimize and predict the properties of specific molten salt mixtures being considered for current nuclear reactor applications.

In general, the MSR Campaign will focus on binary, ternary and quaternary salt mixtures because the melting points of individual salts are too high for coolant/fuel applications. A number of appropriate chloride and fluoride salt systems with eutectic behavior have been identified for use as coolants and fuel in different types of nuclear reactors (Table 1 and references cited therein). However, there remains a need for a functional database that can support salt mixture properties modeling over the full range of relevant P-T-X (pressure, temperature, composition) conditions to optimize and assess the performance of MSR designs.

\subsection{DATA NEEDS AND PRIORITIZATION}

A coolant or fuel-coolant salt must efficiently extract heat from the core at relatively high temperatures and efficiently transfer that heat to heat exchangers and energy conversion systems (e.g., steam generator- 
turbine system) without introducing unmanageable safety or waste issues. Examples of some of the key requirements include:

- Neutron attenuation: the salt should not significantly attenuate neutrons.

- Activation: the salt should not produce significant quantities of radioactive activation products that could complicate regular operation tasks (low neutron absorption cross sections) or substantially increase the source term.

- Corrosion: the salt should not be aggressively corrosive to stainless steels or other alloys used for core or loop components.

- Actinide solubility: the solubility of uranium, thorium and plutonium in a candidate fuel-coolant salt should be understood and predictable over relevant temperature ranges.

- Salt freezing: the salt must have a melting point that is at least $50^{\circ} \mathrm{C}$ lower than the lowest temperature that will occur within the coolant loop circuit.

- Salt decomposition or boiling: the salt must be thermally stable at temperature well above those anticipated during normal and off-normal operation (i.e., under credible accident conditions).

- Pumpability: the salt should have a reasonably low viscosity at operating temperatures to allow predictable flow dynamics.

- Vaporization: the salt should have a relatively low vapor pressure to avoid pressurization, aerosol generation and coolant loss issues at temperature.

- Cost and materials availability: salt mixture components should be relatively inexpensive and should be readily available to accommodate scale up and commercialization.

- Opacity: the salt should ideally have low opacity compatible with optical in-service inspection of core and coolant loop components.

- Toxicity: the coolant chemistry should not pose unnecessary toxicity hazard during handling for routine repair operations.

In addition to these general considerations, [24]identify the following specific qualities as essential criteria for assessing the applicability of molten salt systems for nuclear applications.

- Chemical stability of salt mixture at elevated temperatures $\left(500-800^{\circ} \mathrm{C}\right)$.

- Radiolytic stability of salt mixture in high radiation fields (specific to primary coolant).

- Low freezing temperature, preferably lower than $500^{\circ} \mathrm{C}$.

- Large heat capacity and thermal conductivity.

- Low vapor pressures: less than one atmosphere at operating temperatures.

- Compatible with reactor core/heat exchanger materials: alloys, graphite, and ceramics.

Fuel-containing salts have added performance criteria because, in addition to carrying the fissile and fertile material, the salt may be required to act as a neutron moderator, provide negative thermal reactivity feedback, act as the primary heat transfer medium and provide decay heat removal by natural circulation [25]. Therefore, in addition to the requirements listed above, fuel salts also have the following requirements:

- Very low concentrations of isotopes with high-parasitic neutron absorption cross sections within the relevant spectral region for a given reactor.

- Large coefficient of thermal expansion to provide strong negative reactivity feedback and efficient natural circulation.

- Ability to maintain high dissolved concentrations of fissile and fertile materials ( $\mathrm{U}, \mathrm{Pu}, \mathrm{Th})$ to support criticality and efficient breeding, if needed. 
The development of screening factors or operational criteria for salt systems will depend on the specific application within the reactor system. For example, previous studies have suggested that chloride salts are problematic for fast reactor applications due to their relatively high thermal-neutron absorption cross sections and undesirable neutron activation properties (e.g., generation of ${ }^{36} \mathrm{Cl}$ ) $[10,18,19]$. However, recent work indicates that chloride salt systems such as $\mathrm{NaCl}-\mathrm{MgCl}_{2}$ containing fertile ${ }^{238} \mathrm{UCl}_{3}$ and fissile ${ }^{239} \mathrm{PuCl}_{3}$ show considerable promise as a fuel-coolant salt for fast breeder molten salt reactors $[22,26]$.

An example of an application-specific criterion is given by [18], who states that a minimum steady-state temperature for heat transfer in the NGNP/NHI loop is around $680{ }^{\circ} \mathrm{C}$ and that acceptable salt melting points for heat transfer salts must be $454{ }^{\circ} \mathrm{C}$. Also, for relatively high temperature applications [20] indicates that salt coolants should have boiling points around $1300{ }^{\circ} \mathrm{C}$ to ensure stability in the event of thermal excursions during reactor operation.

A wealth of knowledge on the thermophysical properties of molten salts was gained at Oak Ridge National Laboratory in the 1950s - 1970s during the Aircraft Reactor Experiment (ARE), Molten Salt Reactor Experiment (MSRE) and the Molten Salt Breeder Reactor (MSBR) projects [10, 18, 19]. More recently, work on the Advanced High-Temperature Reactor (AHTR), Next Generation Nuclear Plant (NGNP) and Nuclear Hydrogen Initiative (NHI) have provided important information about molten salt property criteria for nuclear applications [17-19]. Other key information on molten salt applications as coolants and fuels in MSRs are compiled in the European Molten Salt Technology (MOST) database [17$19,27]$ and work in Russia described by [16, 28, 29]. Further general salt properties information was made available online through the Molten Salt Database (MSDB) project

(http://allen.neep.wisc.edu/shell/), which was a collaborative effort involving the University of Wisconsin and Shell Oil Company.

In addition to these nuclear application-focused databases, Janz and others compiled and maintained an extensive database of thermophysical and thermochemical property values for molten salts relevant for energy storage [30-40]. The Janz database includes a number of salt compositions that may be applicable as MSR coolants, but actinide-bearing salt compositions are not represented.

Table 2 and Table 3 give melting point, boiling point, vapor pressures, densities, heat capacities, viscosities and thermal conductivities for some of the major candidate molten salt systems. The cells identified as "Data Need" indicate information gaps for these salt compositions. It is likely that new experimental work will be needed to fill these information gaps to further consider use of these mixtures. As pointed out by Williams [18, 19], melting points, vapor pressures and viscosities can be measured accurately, but it is difficult to predict these property values accurately by using current parameter databases. There is a particular need for high temperature heat capacity, viscosity and thermal conductivity data for many relevant salt systems to establish accurate equations for temperature dependencies to predict values throughout the range of operating temperatures.

Table 4 lists a number of possible MSR fuel salts that have been the subject of recent thermodynamic studies by the European Commission's Institute for Transuranium Elements (ITU). For example, Capelli, [41]reports a number of advances in measuring thermophysical and thermochemical properties of MSRrelevant salt mixtures at elevated temperatures.

Table 4 summarizes the results for salt systems that are discussed in some detail by Capelli [41]. 
Table 2 Thermophysical properties extracted from literature for MSR-relevant salts (adapted from [10]).

\begin{tabular}{|c|c|c|c|c|c|}
\hline \multicolumn{2}{|l|}{ Salt Mixture } & \multirow{2}{*}{$\begin{array}{l}\text { Melting } \\
\text { Point or } \\
\text { *Liquidus } \\
\mathrm{T}\left({ }^{\circ} \mathrm{C}\right)\end{array}$} & \multirow{2}{*}{$\begin{array}{l}\text { Boiling } \\
\text { Point } \\
\left({ }^{\circ} \mathrm{C}\right)\end{array}$} & \multirow{2}{*}{$\begin{array}{c}\text { Vapor } \\
\text { Pressure }(\mathrm{mm} \\
\mathrm{Hg}) \text { at } 900^{\circ} \mathrm{C} \\
\text { or Function }\end{array}$} & \multirow[t]{2}{*}{ Density $\left(\mathrm{g} / \mathrm{cm}^{3}\right)$} \\
\hline Type & $\begin{array}{l}\text { Composition } \\
(\mathrm{mol} \%)\end{array}$ & & & & \\
\hline \multirow[t]{4}{*}{$\begin{array}{l}\text { Alkali } \\
\text { Fluorides }\end{array}$} & $\begin{array}{l}\mathrm{LiF}-\mathrm{KF} \\
(50-50) \\
\end{array}$ & 492 & $\begin{array}{l}\text { Data } \\
\text { Need }\end{array}$ & Data Need & $\begin{array}{c}2.460-0.00068 \cdot \mathrm{T}(847- \\
\left.1067^{\circ} \mathrm{C}\right) \\
\end{array}$ \\
\hline & $\begin{array}{l}\text { LiF-RbF } \\
(44-56)\end{array}$ & 470 & $\begin{array}{l}\text { Data } \\
\text { Need }\end{array}$ & Data Need & $\begin{array}{c}3.300-0.00096 \cdot \mathrm{T}(500- \\
\left.750^{\circ} \mathrm{C}\right)\end{array}$ \\
\hline & $\begin{array}{l}\text { LiF-NaF-KF } \\
(46.5-11.5-42)\end{array}$ & 454 & 1570 & -0.7 & $\begin{array}{c}2.530-0.00073 \cdot \mathrm{T}(940- \\
\left.1170^{\circ} \mathrm{C}\right)\end{array}$ \\
\hline & $\begin{array}{l}\text { LiF-NaF-RbF } \\
(42-6-52)\end{array}$ & 435 & $\begin{array}{l}\text { Data } \\
\text { Need }\end{array}$ & -0.8 & $\begin{array}{c}3.261-0.000811 \cdot \mathrm{T}(500- \\
\left.700^{\circ} \mathrm{C}\right)\end{array}$ \\
\hline \multirow[t]{4}{*}{$\mathrm{ZrF}_{4}$ Salts } & $\begin{array}{l}\mathrm{LiF}-\mathrm{ZrF}{ }_{4} \\
(51-49)\end{array}$ & 509 & $\begin{array}{l}\text { Data } \\
\text { Need }\end{array}$ & 77 & $3.739-0.000924 \bullet \mathrm{T}$ \\
\hline & $\begin{array}{l}\mathrm{NaF}-\mathrm{ZrF}_{4} \\
(59.5-40.5)\end{array}$ & 500 & 1350 & 5 & $\begin{array}{c}3.650-0.00088 \cdot \mathrm{T}(550- \\
\left.700^{\circ} \mathrm{C}\right) \\
\end{array}$ \\
\hline & $\begin{array}{l}\mathrm{RbF}-\mathrm{ZrF}_{4} \\
(58-42)\end{array}$ & 410 & 1450 & 1.3 & $3.923-0.00100 \bullet \mathrm{T}$ \\
\hline & $\begin{array}{l}\text { LiF-NaF-ZrF } \\
(26-37-37)\end{array}$ & 436 & $\begin{array}{l}\text { Data } \\
\text { Need }\end{array}$ & 5 & $\begin{array}{c}3.533-0.00087 \cdot \mathrm{T}(500- \\
\left.650^{\circ} \mathrm{C}\right) \\
\end{array}$ \\
\hline \multirow[t]{3}{*}{$\mathrm{BeF}_{2}$ Salts } & $\begin{array}{l}\mathrm{LiF}-\mathrm{BeF}_{2} \\
(67-33)\end{array}$ & 460 & 1400 & Data Need & $\begin{array}{c}2.280-0.000488 \cdot \mathrm{T}(500- \\
\left.650^{\circ} \mathrm{C}\right) \\
\end{array}$ \\
\hline & $\begin{array}{l}\mathrm{NaF}-\mathrm{BeF}_{2} \\
(57-43)\end{array}$ & 340 & 1400 & 1.4 & $\begin{array}{c}2.270-0.00037 \cdot \mathrm{T}(450- \\
\left.550^{\circ} \mathrm{C}\right) \\
\end{array}$ \\
\hline & $\begin{array}{l}\text { LiF-NaF-BeF } \\
(31-31-38)\end{array}$ & 315 & $\begin{array}{l}\text { Data } \\
\text { Need }\end{array}$ & 1.7 & $\begin{array}{c}2.313-0.000450 \bullet \mathrm{T}(450- \\
\left.550^{\circ} \mathrm{C}\right)\end{array}$ \\
\hline \multirow[t]{4}{*}{ Chlorides } & $\begin{array}{l}\mathrm{LiCl}-\mathrm{KCl} \\
(59.5-40.5)\end{array}$ & 355 & 1400 & 5.8 & $1.8772-0.00087 \bullet \mathrm{T}$ \\
\hline & $\begin{array}{l}\mathrm{LiCl}-\mathrm{RbCl} \\
(58-42)\end{array}$ & 313 & $\begin{array}{l}\text { Data } \\
\text { Need }\end{array}$ & Data Need & $2.7416-0.000689 \bullet \mathrm{T}$ \\
\hline & $\begin{array}{l}\text { NaCl-MgCl } \\
(68-32)\end{array}$ & 445 & 1465 & $<2.5$ & $2.2971-0.000507 \bullet \mathrm{T}$ \\
\hline & $\begin{array}{l}\mathrm{KCl}-\mathrm{MgCl}_{2} \\
(68-32)\end{array}$ & 426 & $>1418$ & $<2$ & $2.2546-0.000474 \bullet \mathrm{T}$ \\
\hline \multirow[t]{3}{*}{$\begin{array}{l}\text { Fluoroborate } \\
\text { Salts }\end{array}$} & $\begin{array}{l}\text { NaF-NaBF } 4 \\
(8-92)\end{array}$ & 385 & 694 & 9500 & $2.2521-0.000711 \bullet \mathrm{T}$ \\
\hline & $\begin{array}{l}\mathrm{KF}-\mathrm{KBF}_{4} \\
(25-75)\end{array}$ & 460 & 1070 & 100 & $2.258-0.0008026 \bullet \mathrm{T}$ \\
\hline & $\begin{array}{l}\text { RbF-RbBF } 4 \\
(31-69)\end{array}$ & 442 & 1070 & $<100$ & $2.946-0.001047 \bullet T$ \\
\hline \multirow[t]{2}{*}{$\begin{array}{l}\text { MSR } \\
\text { Breeder } \\
\text { Fuel Salts }\end{array}$} & $\begin{array}{l}\mathrm{LiF}-\mathrm{BeF} 2-\mathrm{ThF} 4- \\
\mathrm{UF}_{4}(73-16-10.7- \\
0.3)\end{array}$ & 500 & $\begin{array}{l}\text { Data } \\
\text { Need }\end{array}$ & $\begin{array}{c}8.0- \\
10000 / \mathrm{T}(\mathrm{K}) \\
\left(500-700^{\circ} \mathrm{C}\right) \\
\end{array}$ & $3.628-0.00066 \cdot \mathrm{T}\left({ }^{\circ} \mathrm{C}\right)$ \\
\hline & $\begin{array}{l}\text { LiF-BeF2-ThF4- } \\
\mathrm{UF}_{4}(72-21-6.7- \\
0.3)\end{array}$ & $500^{*}$ & $\begin{array}{l}\text { Data } \\
\text { Need }\end{array}$ & $\begin{array}{c}8.0- \\
10000 / \mathrm{T}(\mathrm{K}) \\
\left(500-700^{\circ} \mathrm{C}\right) \\
\end{array}$ & $3.153-0.00058 \cdot \mathrm{T}\left({ }^{\circ} \mathrm{C}\right)$ \\
\hline
\end{tabular}




\begin{tabular}{|c|c|c|c|c|c|}
\hline & $\begin{array}{l}\mathrm{LiF}^{-B e F} 2-\mathrm{ThF} 4- \\
\mathrm{UF}_{4}(68-20-11-7- \\
0.3)\end{array}$ & $480^{*}$ & $\begin{array}{l}\text { Data } \\
\text { Need }\end{array}$ & $\begin{array}{c}8.0- \\
10000 / \mathrm{T}(\mathrm{K}) \\
\left(500-700^{\circ} \mathrm{C}\right)\end{array}$ & $3.644-0.00063 \cdot \mathrm{T}\left({ }^{\circ} \mathrm{C}\right)$ \\
\hline & $\begin{array}{l}\text { LiF-BeF2-ThF4- } \\
\mathrm{UF}_{4}(63-25-11.7- \\
0.3)\end{array}$ & $500 *$ & $\begin{array}{l}\text { Data } \\
\text { Need }\end{array}$ & $\begin{array}{c}8.0- \\
10000 / \mathrm{T}(\mathrm{K}) \\
\left(500-700^{\circ} \mathrm{C}\right)\end{array}$ & $3.628-0.00066 \cdot \mathrm{T}\left({ }^{\circ} \mathrm{C}\right)$ \\
\hline
\end{tabular}


Table 3 Additional thermophysical properties extracted from literature for MSR-relevant salts (adapted from [10]).

\begin{tabular}{|c|c|c|c|c|c|c|c|}
\hline \multicolumn{2}{|c|}{ Salt Mixture } & \multicolumn{2}{|c|}{$\begin{array}{l}\text { Heat Capacity } \\
\left(\mathrm{cal} / \mathrm{g}^{\circ} \mathrm{C}\right)\end{array}$} & \multicolumn{2}{|c|}{ Viscosity (cP) } & \multicolumn{2}{|c|}{$\begin{array}{c}\text { Thermal } \\
\text { Conductivity }(\mathrm{W} / \mathrm{m} \\
\mathrm{K})\end{array}$} \\
\hline Type & $\begin{array}{l}\text { Composition } \\
(\mathrm{mol} \%)\end{array}$ & $\begin{array}{c}\text { Measure } \\
\mathrm{d} \\
\left(700^{\circ} \mathrm{C}\right)\end{array}$ & $\begin{array}{c}\text { Predict } \\
\text { ed } \\
\left(700^{\circ} \mathrm{C}\right. \\
)\end{array}$ & $\begin{array}{c}\text { Measure } \\
\mathrm{d} \\
\left(700^{\circ} \mathrm{C}\right)\end{array}$ & T Function & $\begin{array}{l}\text { Predicted } \\
\left(700^{\circ} \mathrm{C}\right)\end{array}$ & $\begin{array}{c}\text { Measure } \\
\mathrm{d}\end{array}$ \\
\hline \multirow{4}{*}{$\begin{array}{l}\text { Alkali } \\
\text { Fluoride } \\
\text { s }\end{array}$} & $\begin{array}{l}\text { LiF-KF } \\
(50-50)\end{array}$ & 0.44 & 0.381 & $\begin{array}{l}\text { Data } \\
\text { Need }\end{array}$ & $\begin{array}{l}\text { Data } \\
\text { Need }\end{array}$ & 0.91 & $\begin{array}{l}\text { Data } \\
\text { Need }\end{array}$ \\
\hline & $\begin{array}{l}\text { LiF-RbF } \\
(44-56)\end{array}$ & 0.284 & 0.226 & $\begin{array}{c}\text { See } \\
\text { Functio } \\
n\end{array}$ & $\begin{array}{c}0.0212 \exp (467 \\
8 / \mathrm{T})(873- \\
1073 \mathrm{~K})\end{array}$ & $T B A$ & $\begin{array}{l}\text { Data } \\
\text { Need }\end{array}$ \\
\hline & $\begin{array}{l}\text { LiF-NaF-KF } \\
(46.5-11.5-42)\end{array}$ & 0.45 & 0.387 & 2.9 & $\begin{array}{c}0.04 \exp (4170 / \\
\mathrm{T})(873- \\
1073 \mathrm{~K})\end{array}$ & 0.92 & $\begin{array}{c}0.6 \\
\left(500^{\circ} \mathrm{C}\right)\end{array}$ \\
\hline & $\begin{array}{l}\text { LiF-NaF-RbF } \\
(42-6-52)\end{array}$ & $\begin{array}{l}\text { Data } \\
\text { Need }\end{array}$ & 0.236 & 2.6 & $\begin{array}{l}\text { Data } \\
\text { Need } \\
\end{array}$ & 0.62 & $\begin{array}{l}\text { Data } \\
\text { Need }\end{array}$ \\
\hline \multirow[t]{4}{*}{$\begin{array}{l}\mathrm{ZrF}_{4} \\
\text { Salts }\end{array}$} & $\begin{array}{l}\mathrm{LiF}-\mathrm{ZrF}_{4} \\
(51-49)\end{array}$ & $\begin{array}{l}\text { Data } \\
\text { Need }\end{array}$ & 0.292 & $>5.1$ & $\begin{array}{l}\text { Data } \\
\text { Need }\end{array}$ & 0.48 & $\begin{array}{l}\text { Data } \\
\text { Need }\end{array}$ \\
\hline & $\begin{array}{l}\mathrm{NaF}-\mathrm{ZrF}_{4} \\
(59.5-40.5)\end{array}$ & 0.28 & 0.275 & 5.1 & $\begin{array}{c}0.0767 \exp (397 \\
7 / \mathrm{T})(873- \\
1073 \mathrm{~K}) \\
\end{array}$ & 0.49 & $\begin{array}{l}\text { Data } \\
\text { Need }\end{array}$ \\
\hline & $\begin{array}{l}\mathrm{RbF}-\mathrm{ZrF}_{4} \\
(58-42)\end{array}$ & $\begin{array}{l}\text { Data } \\
\text { Need }\end{array}$ & 0.2 & 5.1 & $\begin{array}{l}\text { Data } \\
\text { Need }\end{array}$ & 0.39 & $\begin{array}{l}\text { Data } \\
\text { Need }\end{array}$ \\
\hline & $\begin{array}{l}\text { LiF-NaF-ZrF } \\
(26-37-37)\end{array}$ & $\begin{array}{l}\text { Data } \\
\text { Need }\end{array}$ & 0.296 & 6.9 & $\begin{array}{l}\text { Data } \\
\text { Need } \\
\end{array}$ & 0.53 & $\begin{array}{l}\text { Data } \\
\text { Need }\end{array}$ \\
\hline \multirow[t]{3}{*}{$\begin{array}{l}\mathrm{BeF}_{2} \\
\text { Salts }\end{array}$} & $\begin{array}{l}\text { LiF-BeF } \\
(67-33)\end{array}$ & 0.577 & 0.566 & 5.6 & $\begin{array}{c}0.0116 \exp (375 \\
5 / \mathrm{T})(873- \\
1073 \mathrm{~K})\end{array}$ & 1.1 & $\begin{array}{c}1.0 \\
\left(600^{\circ} \mathrm{C}\right)\end{array}$ \\
\hline & $\begin{array}{l}\mathrm{NaF}-\mathrm{BeF}_{2} \\
(57-43)\end{array}$ & 0.52 & 0.44 & 7 & $\begin{array}{c}0.0346 \exp (516 \\
5 / \mathrm{T})(873- \\
1073 \mathrm{~K}) \\
\end{array}$ & 0.87 & 1 \\
\hline & $\begin{array}{l}\text { LiF-NaF-BeF } 2 \\
(31-31-38)\end{array}$ & $\begin{array}{l}\text { Data } \\
\text { Need }\end{array}$ & 0.489 & 5 & $\begin{array}{l}\text { Data } \\
\text { Need }\end{array}$ & 0.97 & $T B A$ \\
\hline \multirow[t]{4}{*}{$\begin{array}{l}\text { Chlorid } \\
\text { es }\end{array}$} & $\begin{array}{l}\mathrm{LiCl}-\mathrm{KCl} \\
(59.5-40.5)\end{array}$ & 0.287 & 0.289 & 1.15 & $\begin{array}{c}0.0861 \exp (251 \\
7 / \mathrm{T})(873- \\
1173 \mathrm{~K}) \\
\end{array}$ & 0.42 & $\begin{array}{c}0.38 \\
\left(700^{\circ} \mathrm{C}\right)\end{array}$ \\
\hline & $\begin{array}{l}\mathrm{LiCl}-\mathrm{RbCl} \\
(58-42)\end{array}$ & 0.213 & 0.212 & 1.3 & $\begin{array}{l}\text { Data } \\
\text { Need }\end{array}$ & 0.36 & $\begin{array}{l}\text { Data } \\
\text { Need }\end{array}$ \\
\hline & $\begin{array}{l}\mathrm{NaCl}-\mathrm{MgCl}_{2} \\
(68-32)\end{array}$ & 0.258 & 0.262 & 1.36 & $\begin{array}{c}0.0286 \exp (144 \\
1 / \mathrm{T})(973- \\
1173 \mathrm{~K}) \\
\end{array}$ & 0.5 & $\begin{array}{l}\text { Data } \\
\text { Need }\end{array}$ \\
\hline & $\begin{array}{l}\mathrm{KCl}-\mathrm{MgCl}_{2} \\
(68-32)\end{array}$ & 0.276 & 0.229 & 1.4 & $\begin{array}{c}0.0146 \exp (223 \\
0 / \mathrm{T})(900- \\
1073 \mathrm{~K})\end{array}$ & $\begin{array}{l}\text { Data } \\
\text { Need }\end{array}$ & $\begin{array}{l}\text { Data } \\
\text { Need }\end{array}$ \\
\hline $\begin{array}{l}\text { Fluorob } \\
\text { orate }\end{array}$ & $\begin{array}{l}\mathrm{NaF}-\mathrm{NaBF}_{4} \\
(8-92)\end{array}$ & 0.36 & 0.435 & 0.9 & $\begin{array}{c}0.0877 \exp (224 \\
0 / \mathrm{T})(873-\end{array}$ & 0.4 & $\begin{array}{c}0.4 \\
\left(621^{\circ} \mathrm{C}\right)\end{array}$ \\
\hline
\end{tabular}




\begin{tabular}{|c|c|c|c|c|c|c|c|}
\hline \multirow[t]{3}{*}{ Salts } & & & & & $1173 \mathrm{~K})$ & & \\
\hline & $\begin{array}{l}{\mathrm{KF}-\mathrm{KBF}_{4}}^{(25-75)}\end{array}$ & 0.312 & 0.367 & 0.9 & $\begin{array}{c}0.0431 \exp (306 \\
0 / \mathrm{T})(873- \\
1073 \mathrm{~K}) \\
\end{array}$ & 0.38 & $\begin{array}{l}\text { Data } \\
\text { Need }\end{array}$ \\
\hline & $\begin{array}{l}\mathrm{RbF}-\mathrm{RbBF}_{4} \\
(31-69)\end{array}$ & 0.218 & 0.258 & 0.9 & $\begin{array}{l}\text { Data } \\
\text { Need }\end{array}$ & 0.28 & $\begin{array}{l}\text { Data } \\
\text { Need }\end{array}$ \\
\hline \multirow{4}{*}{$\begin{array}{l}\text { MSR } \\
\text { Breeder } \\
\text { Fuel } \\
\text { Salts }\end{array}$} & $\begin{array}{l}\mathrm{LiF}-\mathrm{BeF}_{2}-\mathrm{ThF}_{4^{-}} \\
\mathrm{UF}_{4}(73-16-10.7- \\
0.3)\end{array}$ & 0.34 & $\begin{array}{l}\text { Data } \\
\text { Need }\end{array}$ & $\begin{array}{l}\text { Data } \\
\text { Need }\end{array}$ & $\begin{array}{c}0.084 \exp (4340 / \\
\mathrm{T})\end{array}$ & 0.01 & $\begin{array}{l}\text { Data } \\
\text { Need }\end{array}$ \\
\hline & $\begin{array}{l}\mathrm{LiF} \mathrm{BeF}_{2}-\mathrm{ThF}_{4^{-}} \\
\mathrm{UF}_{4}(72-21-6.7- \\
0.3)\end{array}$ & 0.39 & $\begin{array}{l}\text { Data } \\
\text { Need }\end{array}$ & $\begin{array}{l}\text { Data } \\
\text { Need }\end{array}$ & $\begin{array}{c}0.072 \exp (4370 / \\
\mathrm{T})\end{array}$ & 0.01 & $\begin{array}{l}\text { Data } \\
\text { Need }\end{array}$ \\
\hline & $\begin{array}{l}\mathrm{LiF}_{-\mathrm{BeF}_{2}-\mathrm{ThF}_{4-}} \\
\mathrm{UF}_{4}(68-20-11-7- \\
0.3)\end{array}$ & 0.33 & $\begin{array}{l}\text { Data } \\
\text { Need }\end{array}$ & $\begin{array}{l}\text { Data } \\
\text { Need }\end{array}$ & $\begin{array}{c}0.044 \exp (5030 / \\
\mathrm{T})\end{array}$ & 0.007 & $\begin{array}{l}\text { Data } \\
\text { Need }\end{array}$ \\
\hline & $\begin{array}{l}\mathrm{LiF} \mathrm{BeF}_{2}-\mathrm{ThF}_{4^{-}} \\
\mathrm{UF}_{4}(63-25-11.7- \\
0.3)\end{array}$ & 0.34 & $\begin{array}{l}\text { Data } \\
\text { Need }\end{array}$ & $\begin{array}{l}\text { Data } \\
\text { Need }\end{array}$ & $\begin{array}{c}0.084 \exp (4340 / \\
\mathrm{T})\end{array}$ & 0.01 & $\begin{array}{l}\text { Data } \\
\text { Need }\end{array}$ \\
\hline
\end{tabular}

Table 4 Fuel salt compositions studied by Capelli [41].

\begin{tabular}{|c|c|c|c|c|}
\hline Salt Mixture & Composition (mol\%) & $\begin{array}{c}\text { Liquidus } \\
\text { T }\left({ }^{\circ} \mathrm{C}\right)\end{array}$ & $\begin{array}{l}\text { Boiling T } \\
\quad\left({ }^{\circ} \mathrm{C}\right)\end{array}$ & $\begin{array}{c}\text { Vapor } P \text { at } 50 K \\
\text { above Melting } T \\
(\mathrm{~Pa})\end{array}$ \\
\hline $\mathrm{LiF}-\mathrm{ThF}_{4}$ & $76.2-23.8$ & 559 & 1754 & $8.56 \times 10^{-4}$ \\
\hline $\mathrm{Li}-\mathrm{ThF}_{4}-\mathrm{UF}_{4}-\mathrm{PuF}_{3}$ & $78.0-16.0-1.0-5.0$ & 594 & 1762 & $5.33 \times 10^{-3}$ \\
\hline $\mathrm{LiF}-\mathrm{ThF}_{4}-\mathrm{UF}_{4}-\mathrm{PuF}_{3}$ & $75.3-20.6-1.0-3.1$ & 546 & 1759 & $7.26 \times 10^{-4}$ \\
\hline $\mathrm{LiF}-\mathrm{ThF}_{4}-\mathrm{UF}_{4}$ & $76.2-21.3-2.55$ & 557 & 1749 & $1.08 \times 10^{-3}$ \\
\hline $\mathrm{LiF}-\mathrm{BeF}_{2}-\mathrm{ThF}_{4}-\mathrm{UF}_{4}$ & $75.7-1.8-19.95-2.55$ & 552 & 1718 & $4.67 \times 10^{-3}$ \\
\hline $\begin{array}{l}\text { LiF-NaF-ThF }{ }_{4}-U_{4} \\
\text { LiF-NaF-BeF }_{2}-\mathrm{ThF}_{4}-\end{array}$ & $47.3-25.9-24.3-2.55$ & 497 & 1765 & $7.27 \times 10^{-5}$ \\
\hline $\mathrm{UF}_{4}$ & $41.8-31.1-4.6-19.95-2.55$ & 480 & 1706 & $4.08 \times 10^{-5}$ \\
\hline $\mathrm{LiF}-\mathrm{CaF}_{2}-\mathrm{ThF}_{4}$ & 66.9-4.3-28.8 & 538 & 1744 & $4.12 \times 10^{-4}$ \\
\hline
\end{tabular}

Melting point and phase composition information can be readily extracted from accurate binary and ternary phase diagrams (examples shown in Figure 5). Therefore, existing phase diagrams for relevant salt systems will be compiled, catalogued and indexed as part of the data compilation for this new molten salt database. The thermodynamic constants and parameters needed to construct important phase diagrams will also be complied as part of this study (see Section 1 of this report). 

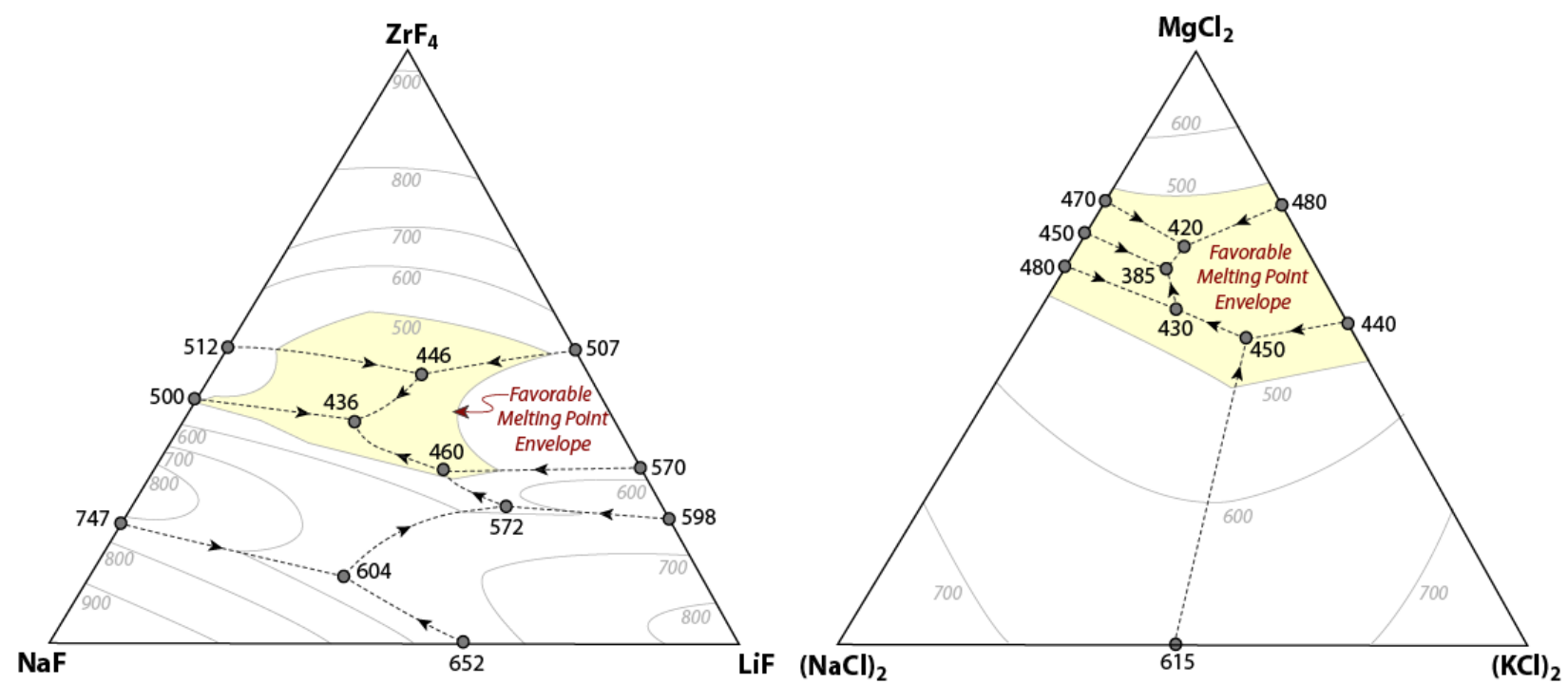

Figure 5 Liquidous surface for the ternary salts $\mathrm{LiF}-\mathrm{NaF}-\mathrm{ZrF}_{4}$ and $\mathrm{NaCl}-\mathrm{KCl}-\mathrm{MgCl}_{2}$ showing temperature contours (light gray), and eutectic points (dark gray points) in ${ }^{\circ} \mathrm{C}$. The yellow region represents compositions that fall within the favorable melting point envelope, making them potential candidates as coolant salts.

The following sections provide recommended methods for modeling property values when experimental results at the condition of interest are lacking by interpolation or extrapolation. A summary of the most widely used thermophysical property models is provided in Table 5. This table forms the basis for future database development activities focused on determining predictive relationships between salt properties and salt temperature and composition.

Table 5 Summary of theoretical and empirical predictive relationships for key salt properties. Each equation is discussed, and terms defined in more detail in the sections below.

\begin{tabular}{|l|l|l|}
\hline Density & $\rho_{\text {mixture }}=\frac{\sum X_{i} M_{i}}{\sum X_{i} V_{i}(T)}$ & Equation 2-1 \\
\hline Heat Capacity & $C_{p}=33.49 * \frac{\sum X_{i} N_{i}}{\sum X_{i} M_{i}}$ & Equation 2-2 \\
\hline Viscosity & $\mu=A e^{-\frac{B}{T}}$ & Equation 2-3 \\
\hline Viscosity & $\mu_{\text {idea mixture }}=\left[\sum\left(X_{i} \mu_{i}^{1 / 3}\right)\right]^{3}$ & Equation 2-4 \\
\hline Thermal Conductivity & $k=0.119 * \frac{T^{0.5} v^{0.667}}{(M / n)^{1.167}}$ & Equation 2-5 \\
\hline Thermal Conductivity & $k=5.0 \times 10^{-4} T+\frac{32.0}{M}-0.34$ & Equation 2-6 \\
\hline
\end{tabular}


$\rho_{\text {mixture }}$ - density of the molten salt mixture $\left(\mathrm{g} / \mathrm{cm}^{3}\right), X_{i}$ - mole fraction of component $i$, $M_{i}$ - molecular weight of component $i\left(\mathrm{~g} /\right.$ mole),$V_{i}(T)$ - molecular volume of component $i$ at temperature $T, N_{i}$ - number of atoms per salt component $i, \mu$ - dynamic viscosity in centipoise (cP), A and B are empirical constants, $k$ - thermal conductivity in watt $/ \mathrm{m} \mathrm{K}$, $T_{m}$ - salt mixture melting point in $\mathrm{K}, v$ - molar of the salt $\left(\mathrm{cm}^{3} / \mathrm{mol}\right), M$ - average formula weight of the salt, $n$ - number of ions per salt formula.

In addition to the properties reviewed below, some databases (e.g., reference [35]) include properties that are less commonly referenced but may be important for developing fundamental relationships between salt chemistry and thermophysical behavior, such as surface tension, electrical conductivity, bulk modulus, self-diffusivity of components and cryoscopic constants. These properties can be screened to determine if they provide essential functional needs within the thermophysical properties database to be integrated with MSR models.

\subsection{MELTING/FREEZING POINT}

In a critical review of coolant salts for the AHTR [19], identifies the melting (freezing) point as the single most important thermophysical property for a candidate salt system. The freezing point threshold for a given candidate coolant depends on the system design; it is generally accepted that the coolant salt will need to be in the molten state at temperatures above approximately $500{ }^{\circ} \mathrm{C}[18,19]$. Two important criteria that need to be defined are the safety margin for the freezing point of a given coolant salt for a given reactor concept and the applicable grace period for the onset of freezing that can be tolerated under a given transient scenario. For example, $[18,19]$ indicates a grace period for the onset of freezing of around 70 minutes for an off-site power loss scenario and a 5-7 minute grace period for a loss of heat sink transient.

Williams provides critical reviews $[18,19]$ of relevant ternary systems which map relevant melting point compositional envelopes (contoured liquidous surface) (see Figure 5 above) and discuss why some low melting point compositions are considered promising coolant candidates while others are not. For example, it has been observed that low melting point $\mathrm{BeF}_{2}$-rich salts become exceedingly viscous due to the associative behavior of $\mathrm{Be}^{2+}$ in a molten fluoride. Another example is the well-studied LiF-NaF- $\mathrm{ZrF}{ }_{4}$ system, which has the fairly broad and favorable melting point envelope shown in Figure 5. As pointed out by Williams [18, 19], compositions with more than $40 \mathrm{~mol} \% \mathrm{ZrF}_{4}$ are not favored as coolants due to the significant vapor pressure imparted by $\mathrm{ZrF}_{4}$ at higher concentrations. The point here is that not all salt compositions within the favorable melting point envelope are necessarily good candidates for use as coolant salt- other screening properties must be considered.

\subsection{VAPOR PRESSURE}

As shown by Williams $[18,19]$, binary and ternary mixtures of salts exhibit lower vapor pressures (higher boiling points) than their constituent, pure salt components. This allows for the selection of multicomponent salts with low melting points and low vapor pressures within the operational range of $500{ }^{\circ} \mathrm{C}-800{ }^{\circ} \mathrm{C}$. However, as mentioned above, other thermophysical and chemical factors may determine the suitability of a given salt system as a coolant.

The vapor pressures and boing points of salt mixtures may be the second most important property for screening potential coolant salts [10]. Forsberg [42] suggests that coolant salts should have boiling points high enough to preclude boiling of the coolant even under reactor operational transients (e.g., 
temperatures as high as $\left.1300{ }^{\circ} \mathrm{C}[10]\right)$. There are several studies focused on the vapor pressures and vapor speciation for relevant salt systems in the literature [14, 43, 44]. Vapor pressures for fuel salts considered for breeder reactor concepts are discussed by in references [45, 46]. The vapor pressures of several key salt mixtures were shown in Table 2 and Table 4 above.

For the MSR campaign modeling efforts, it is anticipated that vapor pressures and vapor phase speciation for fuel-salts and coolant salts will be calculated in the thermochemical solver by using the thermochemical databased discussed in Section 1 above. However, experimental vapor pressure measurements as well as empirical vapor pressure models will be compiled as part of the thermophysical database development activity to validate the thermodynamic calculations.

\subsection{DENSITY}

The coolant density as a function of temperature plays a key role in heat transport capability for both natural convection and forced convection systems. Density is readily measured and can be accurately predicted for unknown compositions by using established methods [14, 47]. By comparing measured and calculated values, $[18,19]$, showed that this additive molar volume method predicts salt densities to an accuracy of better than 5\%. The recommended formulation of this method is as follows:

$\rho_{\text {mixture }}=\frac{\sum X_{i} M_{i}}{\sum X_{i} V_{i}(T)}$

where $\rho_{\text {mixture }}$ is the density of the molten salt mixture $\left(\mathrm{g} / \mathrm{cm}^{3}\right), X_{i}$ is the mole fraction of component $i$, $M_{i}$ is the molecular weight of component $i(\mathrm{~g} /$ mole $)$ and $V_{i}(T)$ is the molecular volume of component $i$ at temperature $T$.

In general, salts for which density changes significantly with temperature may be favored for use as coolants due to their greater capacity to remove heat by natural convection relative to salts with densities that are less sensitive to temperature. However, salts with high densities may become problematic for some pumping equipment $[18,19]$. Overall, density is a key property for modeling coolant behavior but is not a salt selection criterion.

\subsection{HEAT CAPACITY}

Molten salts have relatively large heat capacities, and some of the fluoride mixtures have a heat carrying capacity similar to water if density differences are taken into account $[18,19]$. First principles methods for predicting heat capacities for novel salt compositions are not fully developed, but there are some established empirical relationships that can be used for estimations. These relationships generally involve estimating the heat capacity of the salt mixture based on mole fraction-weighted contributions from purecomponents and accounting for mixing enthalpies where available $[18,19]$.

For example, $[18,19]$, assert that the "Dulong and Petit" method, which assumes a contribution of 33.49 $\mathrm{J} /{ }^{\circ} \mathrm{C}$ per mole of each atom in the mixture, is the most successful heat capacity predictive function. The Dulong-Petit approximation can be expressed as follows:

$C_{p}=33.49 * \frac{\sum X_{i} N_{i}}{\sum X_{i} M_{i}}$ 
where $X_{i}$ is the mole fraction of component $i, N_{i}$ is the number of $i$ atoms per formula salt component (e.g., 2 for alkali halides, 3 for $\mathrm{BeF}_{2}, 5$ for $\mathrm{ZrF}_{4}$ ), and $M_{i}$ is the molecular weight of component $i(\mathrm{~g} / \mathrm{mole}$ ).

Based on calculations by [19] the Dulong-Petit method is generally accurate to within $10 \%$ for $\mathrm{BeF}_{2}$ and $\mathrm{ZrF}_{4}$ bearing salts and to within $20 \%$ for other AHTR coolant candidate salts (Table 6). There is a lack of temperature dependent heat capacity data for many relevant salt compositions, but [19]notes that, in general, salt mixture heat capacities do not vary considerably over the temperature ranges relevant for MSR designs.

Table 6 Comparison of measured heat capacities for candidate coolant salts with values calculated using the Dulong-Petit model (adapted from [18, 19]).

\begin{tabular}{|c|c|c|c|}
\hline Salt & $\begin{array}{c}\text { Composition (mol } \\
\%)\end{array}$ & $\begin{array}{l}\mathrm{C}_{\mathrm{p}} \text { measured at } \\
7_{000^{\circ} \mathrm{C}\left(\mathrm{J} / \mathrm{g}^{\circ} \mathrm{C}\right)}\end{array}$ & $\begin{array}{c}\mathrm{C}_{\mathrm{p}} \text { predicted by } \\
\text { Dulong-Petit model } \\
\left(\mathrm{J} / \mathrm{g}^{\circ} \mathrm{C}\right)\end{array}$ \\
\hline Li-NaF-KF & 46.5-11.5-42 & 1.88 & 1.62 \\
\hline LiF-KF & $50-50$ & 1.84 & 1.59 \\
\hline LiF-NaF-Rb & $42-6-52$ & No Data & 0.99 \\
\hline LiF-RbF & $43-57$ & 1.19 & 0.95 \\
\hline $\mathrm{LiF}-\mathrm{BeF}_{2}$ & $66.7-33.3$ & 2.41 & 2.37 \\
\hline $\begin{array}{l}\mathrm{NaF}_{-} \mathrm{BeF}_{2} \\
\mathrm{LiF}-\mathrm{NaF}-\end{array}$ & $57-43$ & 2.18 & 1.84 \\
\hline $\mathrm{BeF}_{2}$ & $31-31-38$ & No Data & 2.05 \\
\hline $\begin{array}{l}\text { LiF-ZrF } \\
\text { LiF-NaF- }\end{array}$ & $51-49$ & No Data & 1.22 \\
\hline $\mathrm{ZrF}_{4}$ & $26-37-37$ & No Data & 1.24 \\
\hline $\mathrm{NaF}-\mathrm{ZrF}_{4}$ & $57-43$ & 1.17 & 1.15 \\
\hline $\mathrm{KF}-\mathrm{NaF}-\mathrm{ZrF}{ }_{4}$ & $52-5-43$ & 1.09 & 1.05 \\
\hline $\mathrm{KF}-\mathrm{ZrF}_{4}$ & $58-42$ & No Data & 1.05 \\
\hline $\mathrm{RbF}-\mathrm{ZrF}_{4}$ & $58-42$ & No Data & 0.84 \\
\hline LiCl-KCl & $59-41$ & 1.20 & 1.21 \\
\hline LiCl-RbCl & $58-42$ & 0.89 & 0.89 \\
\hline $\mathrm{NaCl}-\mathrm{MgCl}_{2}$ & $58-42$ & 1.08 & 1.10 \\
\hline $\mathrm{KCl}-\mathrm{MgCl}_{2}$ & $67-33$ & 1.15 & 0.96 \\
\hline $\mathrm{NaF}_{-\mathrm{NaBF}_{4}}$ & $8-92$ & 1.51 & 1.82 \\
\hline $\mathrm{KF} \mathrm{KBF}_{4}$ & $25-75$ & 1.31 & 1.54 \\
\hline $\mathrm{RbF} \mathrm{RbBF}_{4}$ & $31-69$ & 0.91 & 1.08 \\
\hline
\end{tabular}

\subsection{VISCOSITY}

The resistance to flow of a salt mixture, as quantified by viscosity, is another important property for modeling heat transport and fluid dynamics [10]. Viscosity is temperature dependent and increases exponentially with decreasing temperature, depending on salt composition (e.g., Table 3). Samuel [10] states that, as a general rule, a coolant salt viscosity should be below 10 centipoise at operational temperatures. 
Molten salts are Newtonian fluids that show an exponential decrease in viscosity with reciprocal temperature $[18,19]$. This dependence can be expressed as follows:

$\mu=A e^{-\frac{B}{T}}$

where $\mu$ is the dynamic viscosity in centipoise (cP), T is temperature in Kelvin, and A and $\mathrm{B}$ are empirical constants.

Based on this exponential relationship, viscosity changes more with temperature than do the other key thermophysical salt properties $[18,19]$. There are no established methods for estimating the empirical constants needed for predicting viscosity as a function of temperature and viscosity values for candidate coolant and fuel-coolant salts must be determined experimentally.

For some salt compositions lacking experimental data, it may be appropriate to estimate viscosity based on a mole fraction weighted sum (Equation 2-4 below). However, this approach is only valid if the salt closely approximates an ideal mixture.

$\mu_{\text {ideal mixture }}=\left[\sum\left(X_{i} \mu_{i}^{1 / 3}\right)\right]^{3}$

where $X_{i}$ is the mole fraction of component $i$ and $\mu_{i}$ is the dynamic viscosity of component $i$ (centipoise).

Empirical temperature functions for viscosity have been determined for a number of relevant salt systems (e.g., Table 3). However, a cursory critical review shows that systematic discrepancies occur between existing data (e.g., Figure 6). A thorough and comprehensive critical review of viscosity data for relevant salt systems is needed to establish compositionally-dependent trends and bounds, as well as to identify and prioritize data gaps that need to be filled through new experimental work.

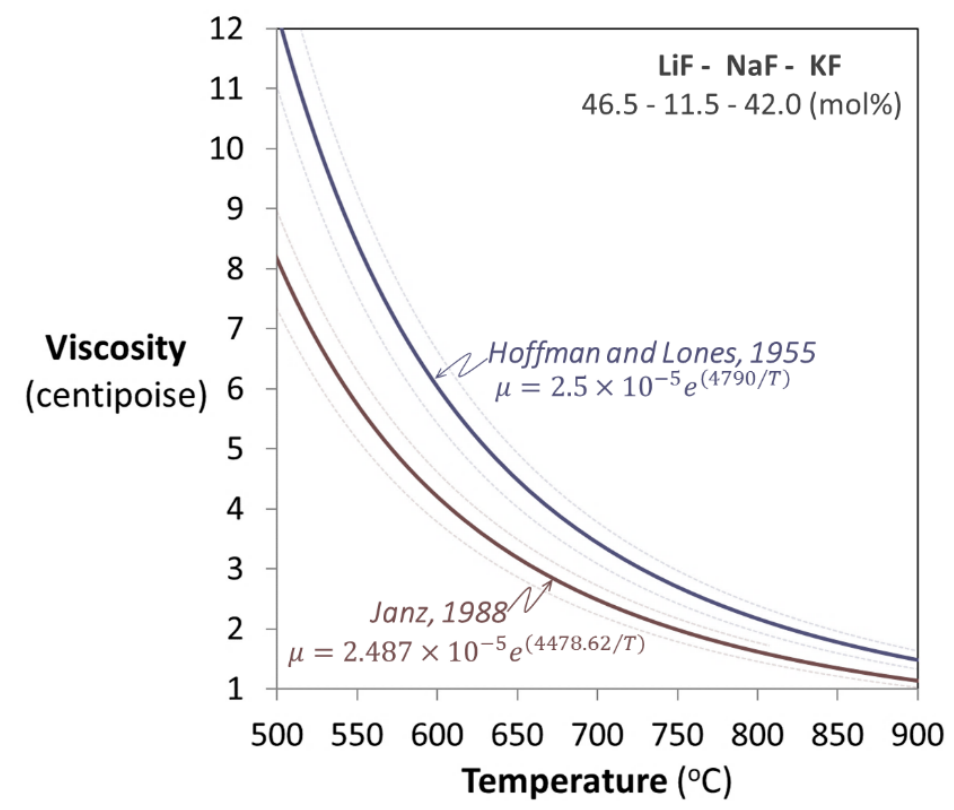

Figure 6 Example of discrepancies between empirical fits to two different viscosity data sets for LiF-NaF-KF.

As with melting point and vapor pressure, viscosity is determined largely by the coordination chemistry of particular species within the melt. For example, beryllium tends to self-associate in fluoride melts that 
lack enough free $\mathrm{F}^{-}$to fully coordinate $\mathrm{Be}^{2+}$. In such mixtures, the self-association of $\mathrm{Be}^{2+}$ creates an extended molecular ordering that increases the viscosity of the molten salt $[18,19]$. The valence of the alkali cation in the salt mixture also plays a role in this effect, as monovalent ions such as $\mathrm{Na}^{+}$and $\mathrm{Rb}^{+}$ give up their coordinating $\mathrm{F}^{-}$ions more readily than divalent cations to minimize $\mathrm{Be}^{2+}$ self-association. Because of this phenomenon, salt mixtures with more than $45 \mathrm{~mol}^{\%} \mathrm{BeF}_{2}$ are generally too viscous to be considered suitable candidates for coolant purposes $[18,19]$.

Williams $[18,19]$ compared viscosity measurements made at ORNL in the 1950s and 1960s by using capillary efflux and rotational viscometry methods to measurements made in the 1990s and 2000s made by using oscillating cup viscometry and found that the older measurements tended to produce values around $10 \%$ higher than the recent measurements. This is not a large discrepancy considering the accuracy of the older measurements was estimated to be around $20 \%$. The observations that the older measurements are consistently higher may be attributed to the fact that the newer methods were customized for high temperature measurements and are more sensitive to low-viscosity fluids [18, 19]. A more in-depth treatment of molten salt viscosity at high temperatures is provided by Veliyulin et al. [48].

It has been observed that viscosities of fluoride salt mixtures vary significantly with salt composition [18, 19, 49]. Examples of how compositional changes in a salt composition can influence viscosity are shown in Figure 7. Specifically, Figure 7a shows the considerable decrease in viscosity for chloride salts relative to fluoride salts and Figure $7 \mathrm{~b}$ shows how an increase in $\mathrm{BeF}_{2}$ and decrease in $\mathrm{Th} \mathrm{F}_{2}$ results in a viscosity decrease of around $2 \mathrm{cP}$ over this temperature range.
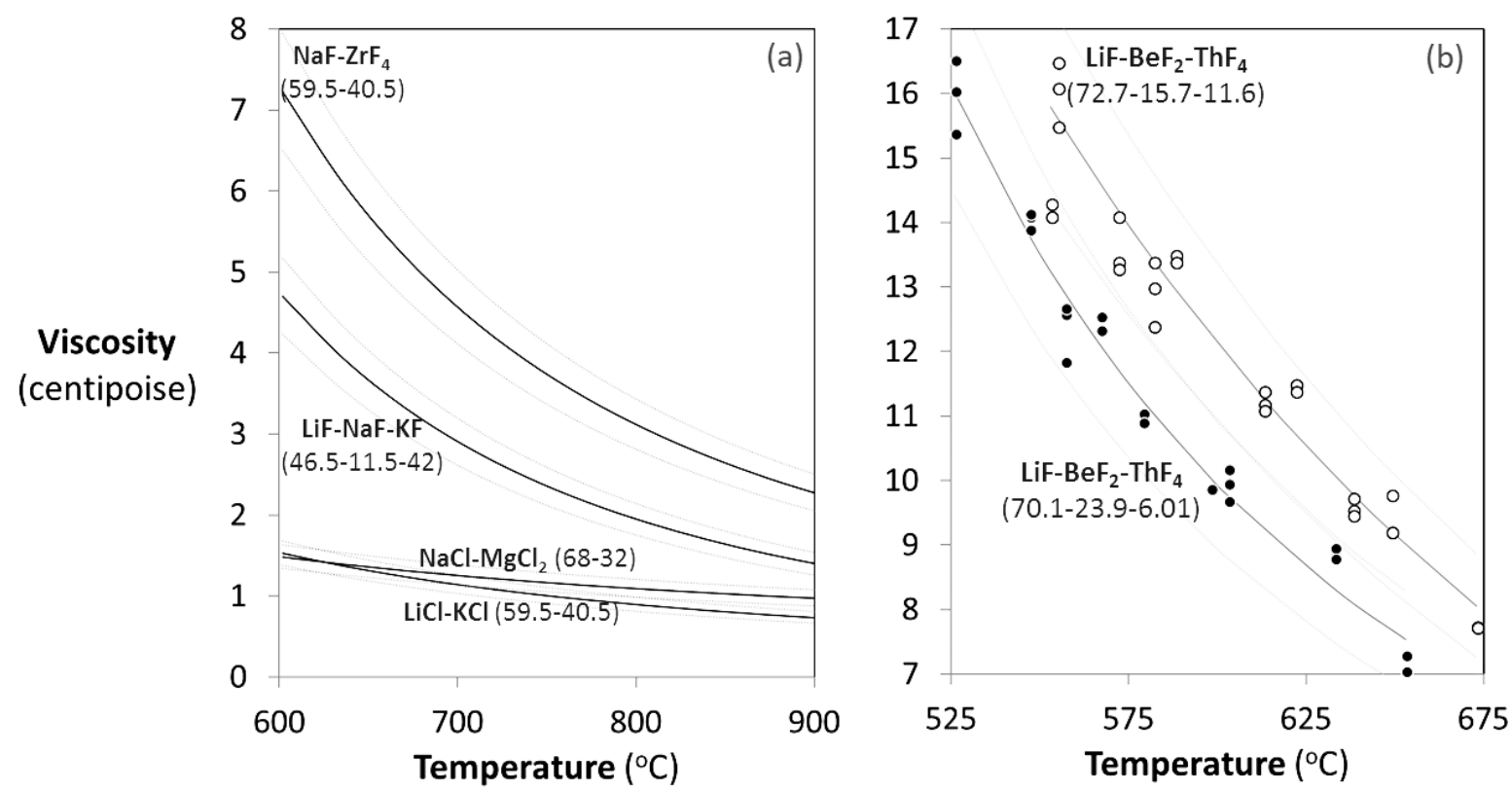

Figure 7 Example datasets showing viscosity as a function of temperature for selected salt compositions. (a) shows empirically derived functions from [18, 19] with 10\% error envelopes, (b) shows measured data points from Cohen and Jones [49] and exponential trendlines with $5 \%$ error envelopes.

\subsection{THERMAL CONDUCTIVITY}

Further experimental validation of both the heat capacities and thermal conductivities of candidate salts is needed $[18,19]$ (see examples in Table 3). Accurate prediction methods for thermal conductivity have been demonstrated $[28,29]$, but there remains a need for significant experimental validation [10]. Thermal conductivity is essential for heat transfer calculations and reliable temperature dependence 
functions are needed for the design of heat exchangers that will be in direct contact with the coolant salt $[10]$.

Williams $[18,19]$ identified thermal conductivity as the most challenging thermophysical property to measure for relevant molten salt compositions at relevant temperatures. Uncertainties in thermal conductivity directly affect the accuracy of heat transfer calculations for reactor design and optimization. For example, early measurements made on relevant salt mixtures by using a variable-gap thermal conductivity apparatus [50]produced values approximately $40 \%$ higher than more recent measurements made for the same salts $[18,19,51,52]$ by using an improved variable-gap apparatus that was customized to minimize errors due to convective heat loss [51]. Recent advances have led to the development of methods for measuring molten salt thermal conductivity that are considerably more accurate, such as the laser flash method [53].

There remain important uncertainties associated with the heat transfer mode of molten salts at high temperatures [23]. Nieto de Castro [54] identified five of the main sources of uncertainties affecting of thermal conductivity measurements for molten salts at high temperature: (1) sample purity and homogeneity, (2) thermal stability of salt, (3) interactions between sample and sample container material and cover gas phase, (4) reliability of thermal sensors, and (5) competing heat transfer mechanisms such as convection and radiation.

The challenging nature of measuring thermal conductivities of molten salts at reactor-relevant temperatures has led to significant scatter in the experimental database for relevant coolant salts $[18,19]$. Despite this difficulty, attempts have been made to derive empirical predictive models as a function of temperature using what are considered the most reliable experimental data $[18,19]$. For example, Cornwell [55] derived the following expression:

$k=0.119 * \frac{T_{m}^{0.5} v^{0.667}}{(M / n)^{1.167}}$

where $k$ is thermal conductivity in watt $/ \mathrm{m} \mathrm{K}, T_{m}$ is the salt mixture melting point in $\mathrm{K}, v$ is the molar volume of the salt $\left(\mathrm{cm}^{3} / \mathrm{mol}\right), M$ is the average formula weight of the salt, and $n$ is the number of ions per salt formula (e.g., 2 for $\mathrm{NaCl})$.

According to Williams $[18,19]$, this model was developed for simple one-component salts (the original formulation lacked the $n$ term) but was extended to mixtures with polyvalent cations by including the ion number $n$. It is anticipated that mixtures will have thermal conductivities below the mole-fraction weighted averages of the single components due to changes in vibrational modes of the salt mixtures coordination "lattice" $[18,19]$.

A simpler empirical correlation for molten salt thermal conductivities measured as part of a Russian program was recommended by Khoklov [56] and Ignatiev et al. [28]:

$k=5.0 \times 10^{-4} T+\frac{32.0}{M}-0.34$

Comparisons of measured values to those calculated using the two expression above are shown in Table 7 (adapted from Williams [18, 19]). 
Table 7 Comparison of measured thermal conductivities with predicted values for candidate coolant salts (adapted from [18, 19]).

\begin{tabular}{|c|c|c|c|c|c|c|}
\hline Salt & $\begin{array}{c}\text { Compositio } \\
\mathbf{n}(\mathrm{mol} \%)\end{array}$ & $\begin{array}{c}\text { Melting } \\
\text { Point }\left({ }^{\circ} \mathrm{C}\right)\end{array}$ & $\begin{array}{c}\text { T of } \\
\text { thermal } \\
\text { conductivit } \\
\mathbf{y}\left({ }^{\circ} \mathrm{C}\right) \\
\end{array}$ & $\begin{array}{c}\text { Measured } \\
(\mathrm{W} / \mathrm{m} \mathrm{K})\end{array}$ & $\begin{array}{c}\text { Cornwell } \\
\text { model, } \\
(\mathrm{W} / \mathrm{m} \mathrm{K}) \\
\end{array}$ & $\begin{array}{c}\text { Kokhlov } \\
\text { model, } \\
(\mathrm{W} / \mathrm{m} \mathrm{K}) \\
\end{array}$ \\
\hline $\mathrm{LiCl}-\mathrm{KCl}$ & $56-41$ & 355 & 355 & 0.69 & 0.65 & 0.82 \\
\hline LiCl-KCl & $56-41$ & 356 & 538 & 0.28 & No Data & No Data \\
\hline LiCl-KCl & $\begin{array}{c}56-41 \\
46.5-11.5-\end{array}$ & 357 & 700 & 0.38 & No Data & No Data \\
\hline LiF-NaF-KF & 42 & 454 & 700 & 0.6 & 0.68 & 0.92 \\
\hline LiF-NaF-RbF & $42-6-52$ & 435 & 700 & No Data & 0.42 & 0.62 \\
\hline $\mathrm{LiF}-\mathrm{BeF}_{2}$ & $66.7-33.3$ & 460 & 600 & 1 & 0.79 & 1.1 \\
\hline $\mathrm{NaF}-\mathrm{BeF}_{2}$ & $57-43$ & 340 & 700 & No Data & 0.58 & 0.87 \\
\hline $\mathrm{LiF}-\mathrm{NaF}-\mathrm{BeF}_{2}$ & $26-37-37$ & 315 & 700 & No Data & 0.62 & 0.97 \\
\hline $\mathrm{LiF}-\mathrm{ZrF}_{4}$ & $51-49$ & 509 & 700 & No Data & 0.35 & 0.48 \\
\hline $\mathrm{NaF}-\mathrm{ZrF}_{4}$ & $59.5-40.5$ & 500 & 700 & No Data & 0.36 & 0.49 \\
\hline $\mathrm{KF}-\mathrm{ZrF}_{4}$ & $58-42$ & 390 & 700 & No Data & 0.32 & 0.45 \\
\hline $\mathrm{RbF}-\mathrm{ZrF}_{4}$ & $58-42$ & 410 & 700 & No Data & 0.26 & 0.39 \\
\hline $\mathrm{LiF}-\mathrm{NaF}-\mathrm{ZrF}_{4}$ & $26-37-37$ & 436 & 700 & No Data & 0.36 & 0.53 \\
\hline $\mathrm{NaF}-\mathrm{AlF}_{3}$ & $75-25$ & 1000 & 1000 & 0.8 & 0.79 & 0.91 \\
\hline
\end{tabular}

Williams $[18,19]$ indicates that accurate thermal conductivities may be obtained for some salt systems by using the simple mole-fraction average of pure component thermal conductivity values. However, this approach should only be used for model development purposes while awaiting reliable experimental values.

\subsection{VOLUME EXPANSION}

Bulk modulus and coefficients of thermal expansion are also important properties needed for predicting and assessing the performance of salts as coolants and fuel coolants in MSRs [57-59]. The work described in these references shows that bulk modulus and thermal expansion (and other properties such as self-diffusivity of components within the salt) can be accurately calculated using molecular dynamics methods because adequate temperature dependent experimental validation data exist.

The volumetric expansion of a coolant salt is another important thermophysical property for assessing candidate salt compositions. Williams [18, 19]compiled the volumetric expansion coefficients from a number of candidate AHTR coolant salts and compared them to those of other coolants used in the nuclear industry: water and sodium. A summary adapted from the Williams [18, 19] compilation is shown in Table 8 below. The average volumetric expansivity of the coolant salts considered is $2.9 \times 10^{-4}$ $\left(1 /{ }^{\circ} \mathrm{C}\right)$ which is a factor of 12 lower than water and around 3 times lower than sodium (Table 8). Other interesting thermophysical comparisons highlighted by Table 8 are that the salt coolants are considerably more dense and viscous than water, have lower heat capacities, but have similar thermal conductivities (properties for water at $300{ }^{\circ} \mathrm{C}$ are compared with salt properties at $700{ }^{\circ} \mathrm{C}$ ). 
Table 8 Comparison of volumetric expansion and other heat transfer properties for several relevant coolants.

\begin{tabular}{|c|c|c|c|c|c|}
\hline Coolant & $\begin{array}{l}\text { Vol. Expansion } \\
\left(1 /{ }^{\circ} \mathrm{C}\right)\end{array}$ & $\begin{array}{l}\text { Heat } \\
\text { Capacity, } \\
\mathrm{Cp}(\mathrm{J} / \mathrm{g} \text { oC }) \\
\end{array}$ & $\begin{array}{l}\text { Density } \\
\left(\mathrm{g} / \mathrm{cm}^{3}\right)\end{array}$ & $\begin{array}{l}\text { Viscosity } \\
\text { (cP) }\end{array}$ & $\begin{array}{l}\text { Thermal } \\
\text { conductivity } \\
(\mathrm{W} / \mathrm{m} \mathrm{K})\end{array}$ \\
\hline Water at $300^{\circ} \mathrm{C}$ & $3.3 \times 10^{-3}$ & 5.73 & 0.72 & 0.09 & 0.54 \\
\hline $\mathrm{Na}$ at $550^{\circ} \mathrm{C}$ & $8.6 \times 10^{-3}$ & 1.27 & 0.82 & 0.23 & 62 \\
\hline \multicolumn{6}{|c|}{ Coolants at $700^{\circ} \mathrm{C}$} \\
\hline FLiNaK & $3.61 \times 10^{-4}$ & 1.88 & 2.02 & 2.9 & 0.92 \\
\hline LiF-NaF-RbF & $3.01 \times 10^{-4}$ & 0.99 & 2.69 & 2.6 & 0.62 \\
\hline $2 \mathrm{LiF}-\mathrm{BeF}_{2}$ & $2.52 \times 10^{-4}$ & 2.41 & 1.94 & 5.6 & 1 \\
\hline $\mathrm{NaF}-\mathrm{BeF}_{2}$ & $1.84 \times 10^{-4}$ & 2.18 & 2.01 & 7 & 0.87 \\
\hline LiF-NaF-BeF ${ }_{2}$ & $2.25 \times 10^{-4}$ & 2.05 & 2 & 5 & 0.97 \\
\hline $\mathrm{LiF}-\mathrm{ZrF}_{4}$ & $2.99 \times 10^{-4}$ & 1.22 & 3.09 & $>5.2$ & 0.48 \\
\hline $\mathrm{NaF}-\mathrm{ZrF}_{4}$ & $2.96 \times 10^{-4}$ & 1.17 & 3.14 & 5.1 & 0.49 \\
\hline $\mathrm{KF}-\mathrm{ZrF}_{4}$ & $3.17 \times 10^{-4}$ & 1.05 & 2.8 & $<5.1$ & 0.45 \\
\hline $\mathrm{RbF}-\mathrm{ZrF} 4$ & $3.11 \times 10^{-4}$ & 0.84 & 3.22 & 5.1 & 0.39 \\
\hline $\mathrm{LiF}-\mathrm{NaF}-\mathrm{ZrF} \mathrm{F}_{4}$ & $3.12 \times 10^{-4}$ & 1.26 & 2.79 & 6.9 & 0.53 \\
\hline $\mathrm{NaF}_{-N a B F}$ & $4.25 \times 10^{-4}$ & 1.51 & 1.75 & 0.88 & 0.5 \\
\hline
\end{tabular}

\subsection{DIMENSIONLESS COEFFICIENTS FOR FLUID DYNAMICS/HEAT TRANSFER MODELING}

A list of dimensionless coefficients used to model heat transfer and fluid dynamics of a salt coolant are given in Table 9. The formulas in this table indicate the relationship between the coefficients and the thermophysical properties discussed above.

Table 9 Key dimensionless coefficients for fluid dynamics.

\begin{tabular}{|l|l|l|}
\hline Coefficient & Description & Formula \\
\hline Nusselt & thermal convection to thermal conduction along $\mathrm{d}_{\mathrm{e}}$ & $\mathrm{Nu}=\mathrm{hd}_{e} / \mathrm{k}$ \\
\hline Reynolds & inertia to viscous forces & $\mathrm{Re}=u L / v$ \\
\hline Prandtl & vicious momentum transfer to thermal conduction & $\mathrm{Pr}=\mathrm{C}_{p} \mu / k$ \\
\hline Grashof & buoyancy to viscous forces & $\mathrm{Gr}=g \mathrm{~L}^{3} \beta \Delta T / v^{2}$ \\
\hline Rayleigh & buoyancy to thermal and viscous momentum transfer & $\mathrm{Ra}=g L^{3} \beta \Delta T / a v$ \\
\hline Froude & inertia to gravitational forces & $\mathrm{Fr}=u^{2} / g L$ \\
\hline Biot & heat transfer at the liquid-solid interface & $\mathrm{Bi}=\mathrm{kl} / \gamma \mathrm{L}$ \\
\hline Strouhal & transient phenomena to convection timescale & $\mathrm{Sr}=\tau u / L$ \\
\hline Fourier & thermal diffusion to convective diffusion of heat & $\mathrm{Fo}=\mathrm{L}^{2} / \alpha \tau$ \\
\hline Weber & inertia to surface forces & $W e=\rho u^{2} L / \sigma$ \\
\hline
\end{tabular}

$h$ : coefficient of heat transfer by convection, or surface conductance, $L$ : fluid characteristic length scale, $u$ : coolant velocity, $d_{e}$ : channel diameter, $k$ : fluid thermal conductivity, $v$ : kinematic viscosity, $\alpha$ : thermal diffusivity, $C_{p}$ : isobaric specific heat, $g$ : acceleration of gravity, $l$ : solid characteristic length scale, $\beta$ : coefficient of thermal expansion, $\gamma$ : coefficient of viscosity linear thermal change, $\mu$ : dynamic viscosity, $\tau$ : timescale. 
Of the coefficients shown in Table 9, the Nusselt, Reynolds and Prandtl numbers are considered the most fundamental in heat transfer models [10]. As indicated in Table 9, the Nusselt number is defined as the ratio of convective heat transfer perpendicular to the direction of the coolant fluid flow to the fluids thermal conductivity [60]. The Reynolds number is the ratio of inertial forces to viscous forces, such that a low Reynolds number corresponds to laminar flow [60]. There is a threshold Reynolds number that marks the transition from laminar to turbulent flow for a given coolant. Thus, high Reynolds numbers characterize turbulent flow involving significant convective heat transfer [60]. The Prandtl number is defined as the ratio of the kinematic viscosity to the thermal diffusivity. The kinematic viscosity quantifies the rate of momentum transfer between molecules and thermal diffusivity is defined as the ratio of heat transfer to the energy stored by the molecules [60]. The only coefficient shown in Table 9 that is temperature and pressure dependent is the Prandtl number. The heat transfer coefficient is dependent on operational conditions and layout such as the coolant channel geometry, flow rate, and system temperature and on other physical properties of the molten salt coolant [10].

In general, molten salts are a category of high Prandtl number fluids that generally show Newtonian behavior $[10,61]$. Based on the available literature, there are a limited amount of experimental measurements of the convective heat transfer in molten salts [10,61]. Experimental work addressing both forced and natural convective flow is required to validate existing molten salt heat transfer models at high temperatures [10]. New experimental work should be conducted to validate application of state-ofthe-art computational fluid dynamics codes to molten salts. The database being compiled as part of the present study aims to provide an up-to-date source of the constants and coefficients needed to support fluid dynamic models for molten salts.

Based on fundamental fluid dynamics equations relating the coefficients in Table 9 and convective heat transfer values for coolants were derived [60], Incorpera and Dewitt [62]. The equations are specific to particular geometries and thermal conditions. The Nusselt number can be used to quantify the convective heat transfer from the coolant [62]It is noted that the equations relating heat transfer coefficients such as those shown in Table 10 need experimental validation for high temperature molten salt systems [10].

Table 10 Convective heat transfer correlations for coolant flow within circular tubes (adapted from Samuel [10]based on equations from El-Wakil [60] and Incorpera, and Dewitt, D. P, 2002).

\begin{tabular}{|l|l|l|}
\hline Correlation Name & Equation & Applicable Conditions \\
\hline Dittus-Boelter & $\begin{array}{l}N u=0.023 \operatorname{Re}^{0.8} \operatorname{Pr}^{n} \\
n=0.4 \text { for heating } \\
n=0.3 \text { for cooling }\end{array}$ & $\begin{array}{l}\text { Isothermal flow neglecting } \\
\text { temperature drop between wall and } \\
\text { bulk fluid. }\end{array}$ \\
\hline Sieder-Tate & $N u=0.023 \operatorname{Re}^{0.8} \operatorname{Pr}^{0.4}\left(\mu_{w} / \mu\right)^{0.14}$ & $\begin{array}{l}\text { Large temperature drop from tube wall } \\
\text { to bulk fluid, } \mu_{w} \text { is the dynamic } \\
\text { viscosity at the tube wall, } \mu \text { is the } \\
\text { dynamic viscosity within the bulk } \\
\text { fluid. }\end{array}$ \\
\hline Colburn & $N u=0.023 \operatorname{Re}^{4 / 5} \operatorname{Pr}^{1 / 3}$ & $\begin{array}{l}\text { For conditions for which Prandtl } \\
\text { numbers range from } 0.5-100 .\end{array}$ \\
\hline
\end{tabular}

An example of such an uncertainty study is provided by Sohal et al.[24] who used the Dittus-Boelter correlation to calculate the uncertainty in the Nusselt number for candidate salt systems based on uncertainties in the thermophysical properties density, viscosity, heat capacity and thermal conductivity. The Nusselt number is the key parameter needed to optimize heat exchanger system designs [10]. Results from the Sohal et al.[24]sensitivity study indicated uncertainties in the Nusselt numbers for Li-F-NaF-KF 
and $2 \mathrm{LiF}-\mathrm{BeF}_{2}$ salts of $8 \%$ and $9 \%$, respectively, and uncertainties as high as $18 \%$ for $\mathrm{KCl}-\mathrm{MgCl}_{2}$, which reflects the elevated uncertainties in the thermophysical properties for the chloride coolant salts [24].

\subsection{FIGURES OF MERIT FOR CANDIDATE SALT COOLANTS}

In an effort to rank coolant performance from a heat-transfer perspective, Williams $[18,19]$ recommend using the figure of merit (FOM) approached discussed by Bonilla [63]. The FOM is specifically defined for the comparative analysis of molten salt coolants based on thermophysical properties. The FOM values are generalized heat-transfer metrics that provide a quantitative means of grouping and ranking coolant performance in the absence of specific reactor design criteria $[18,19]$. Examples of FOM formulas are presented in Table 11. A lower FOM value corresponds to better performance of the salt mixture as a coolant. Thus, the formulas in Table 11 provide means by which thermophysical properties can be combined and used to formulate coolant selection criteria in the absence of a specific reactor and heat exchanger design.

Table 11 Figures of Merit (FOM) equations used in the comparative analysis of molten salt coolant performance depending on flow regime (adapted from Samuel [10]).

\begin{tabular}{|l|l|}
\hline FOM Equation & Flow Regime of FOM \\
\hline$F O M_{1}=\mu^{0.2} /\left(\rho^{2} c_{p}^{2.8}\right)$ & Forced convection, turbulent \\
\hline$F O M_{2}=\left[\mu^{0.2} / \beta\left(\rho^{2} c_{p}^{1.8}\right)\right]^{0.36}$ & Natural convection, turbulent \\
\hline$F O M_{3}=\left[\mu / \beta\left(\rho^{2} c_{p}\right)\right]^{0.36}$ & Natural convection, laminar \\
\hline$F O M_{4}=\mu^{0.2} /\left(\rho^{0.6} c_{p}^{2.8} k^{0.6}\right)$ & Heat exchanger area \\
\hline
\end{tabular}

$\mu$ : viscosity, $\rho$ : fluid density, $C_{p}$ : heat capacity, $\beta$ : volume expansivity, $k$

: thermal conductivity.

Values for the FOMs defined Table 11 for a number of relevant coolants are shown Table 12 (adapted from $[18,19])$. Note that, in general, the "lighter" salt compositions (those not containing $\mathrm{Zr}$, $\mathrm{Rb}$ ) have better heat transfer properties (lower FOM) and that all salts considered are superior to sodium based on this particular viscosity-density-heat capacity-based metric. 
Table 12 Result from example calculations based on feature of merit equations defined in Table 211 above. Coolants are ordered based on values for forced turbulent convection - pumping factor (FOM1).

\begin{tabular}{|c|c|c|c|c|}
\hline & $\begin{array}{l}\mathbf{F O M}_{1} \text {, } \\
\text { Pumping factor }\end{array}$ & $\begin{array}{l}\mathbf{F O M}_{2}, \\
\text { Turbulent }\end{array}$ & $\begin{array}{l}\mathbf{F O M}_{3}, \\
\text { Laminar }\end{array}$ & $\begin{array}{l}\mathbf{F O M}_{4}, \\
\text { Area factor }\end{array}$ \\
\hline Water $\left(300^{\circ} \mathrm{C}\right)$ & 0.2 & 4.8 & 0.6 & 13.0 \\
\hline $2 \mathrm{LiF}-\mathrm{BeF}_{2}$ & 0.7 & 13.9 & 10.1 & 21.5 \\
\hline $\mathrm{NaF}-\mathrm{BeF}_{2}$ & 0.9 & 16.5 & 13.5 & 25.2 \\
\hline $\mathrm{LiF}-\mathrm{NaF}-\mathrm{BeF}_{2}$ & 1.0 & 15.6 & 10.7 & 22.6 \\
\hline LiF-NaF-KF & 1.1 & 13.3 & 6.6 & 21.6 \\
\hline $\mathrm{LiF}-\mathrm{NaF}-\mathrm{ZrF} \mathrm{F}_{4}$ & 1.4 & 13.9 & 9.0 & 35.9 \\
\hline $\mathrm{LiF}-\mathrm{ZrF} \mathrm{F}_{4}$ & 1.8 & 14.5 & 7.9 & 37.5 \\
\hline $\mathrm{NaF}-\mathrm{ZrF}_{4}$ & 2.0 & 14.7 & 7.9 & 37.4 \\
\hline $\mathrm{NaF}_{-} \mathrm{NaBF}_{4}$ & 2.2 & 14.7 & 4.3 & 28.0 \\
\hline $\mathrm{KF}-\mathrm{ZrF}_{4}$ & 3.4 & 16.7 & 9.1 & 42.5 \\
\hline $\mathrm{KF}-\mathrm{KBF}_{4}$ & 3.5 & 15.9 & 4.6 & 35.4 \\
\hline LiF-NaF-RbF & 3.8 & 17.4 & 7.1 & 31.8 \\
\hline $\mathrm{RbF}-\mathrm{ZrF}_{4}$ & 4.8 & 17.6 & 8.9 & 48.7 \\
\hline $\mathrm{KCl}-\mathrm{MgCl}_{2}$ & 5.7 & 21.1 & 7.7 & 39.7 \\
\hline $\mathrm{RbF}^{-\mathrm{RbBF}_{4}}$ & 5.7 & 16.6 & 4.2 & 45.4 \\
\hline LiCl-KCl & 5.9 & 20.8 & 7.2 & 37.5 \\
\hline $\mathrm{NaCl}-\mathrm{MgCl}_{2}$ & 6.4 & 21.7 & 7.8 & 35.1 \\
\hline LiCl-RbCl & 9.0 & 21.3 & 6.9 & 44.5 \\
\hline $\mathrm{Na}$ & 13.2 & 20.3 & 3.5 & 1.6 \\
\hline $\mathrm{Pb}$ & 33.6 & 28.5 & 5.4 & 5.4 \\
\hline
\end{tabular}

\subsection{FISSION PRODUCT CONSIDERATIONS}

In addition to understanding the thermophysical properties of relatively pure binary, ternary and quaternary salt mixtures, there is also a need to develop predictive tools that quantify how salt properties change with time as elements are added to the reactor salt during operations. For example, one of the key questions for the design and optimization of MSR concepts is how the ingrowth of fission products will affect the thermophysical and thermochemical properties of the fuel and coolant salts. Fission products may complicate reactor operation in the following ways $[64,65]$ :

- Unwanted neutron absorption (poisoning).

- Precipitation/plate out in piping leading to increased dose rates thus complicating routine maintenance operations

- Precipitation leading to particulates circulating within the primary coolant

- Volatilization, transport, deposition in cover gas leading to increased dose rates thus complicating routine maintenance operations.

Compere et al. [64] identified isotopes of the following elements as having the highest yields within the MSRE: Sr, Y, Zr, Nb, Mo, Ru, Ag, Sb, Te, I, Cs, Ba, Ce, Nd, Pm, with the relative yields depend strongly of the fissile source $\left({ }^{233} \mathrm{U},{ }^{235} \mathrm{U},{ }^{239} \mathrm{Pu}\right)$. 
Based on chemical behavior, Compere et al. [64] grouped fission products into two broad categories:

- Salt seeking elements that are soluble in fluoride and chloride salts (Rb, $\mathrm{Sr}, \mathrm{Y}, \mathrm{Zr}, \mathrm{Cs}, \mathrm{Ba}, \mathrm{La}, \mathrm{Ce}$, I and other lanthanides)

- Noble elements that are predicted to be insoluble in fluoride and chloride salts $(\mathrm{Nb}, \mathrm{Mo}, \mathrm{Tc}, \mathrm{Ru}$, $\mathrm{Rh}, \mathrm{Pd}, \mathrm{Ag}, \mathrm{Sb}, \mathrm{Te})$.

It is unknown how the buildup of salt-soluble fission products (alkali metals, alkaline earths) will change key thermophysical properties such as melting point, viscosity, heat capacity, and thermal conductivity. This will be one of the main research questions addressed in planned MSR campaign activities to be discussed in Section 5.

\subsection{METHODS, STANDARDS, AND QUALITY ASSURANCE}

A key conclusion drawn from the literature summary presented in Section 2.2 above is that there is a need for the development and documentation of standard methods for the measurement of molten salt thermophysical properties at relevant temperatures $\left(500^{\circ} \mathrm{C}-1000^{\circ} \mathrm{C}\right)$. Systematic discrepancies between datasets and empirical correlations are common throughout the literature for salt compositions that are generally thought of as "well characterized". An example of discrepancies in density data correlations for LiF-NaF-KF is shown in Figure 8.

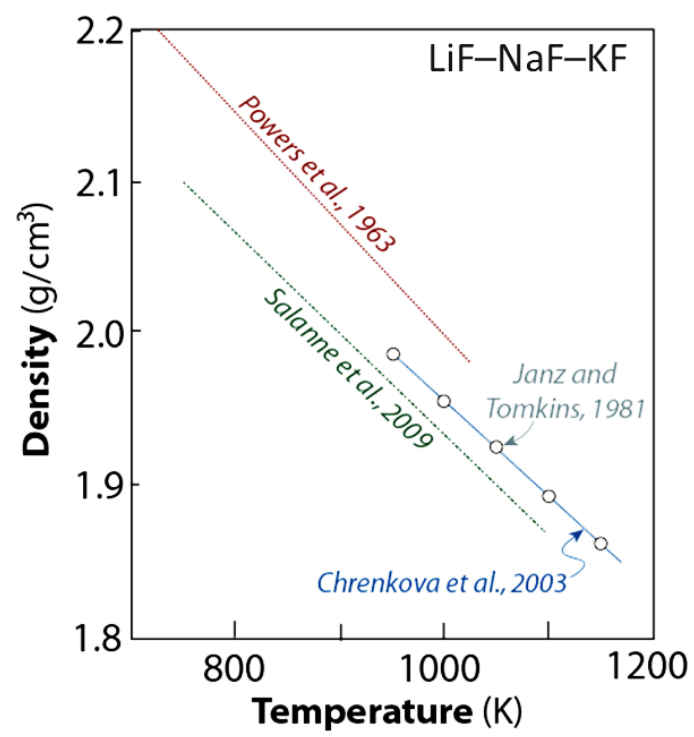

Figure 8 Example of density data from the literature for Li-NaF-KF highlighting discrepancies between measurements.

In an effort to quantify and reduce uncertainties in the new experimental and modeling work performed for the MSR campaign, a program of rigorous quality assurance will be implemented that clearly documents preferred standard methods, best practices and uncertainty quantification requirements.

Because one of the key objectives of the MSR campaign is to produce datasets and modeling and simulation tools that support MSR licensing, having a rigorous QA framework from the outset of the project is paramount. An important part of developing QA rigor is to quantify the uncertainties within the salt properties databases and to establish standard methods and best practices for new experimental 
measurements. Fortner et al. [66] provides an assessment of measurement techniques for key [66]is to identify experimental controls and calibrations that can be used to new thermophysical and thermodynamic measurements provide repeatable results that are reproducible among different analytical laboratories.

The specific topics dealt with in the review of Fortner et al. [66] include the following:

- Fuel salt and coolant salt compositional considerations

- Density measurements:

- Archimedes method

- Gas pycnometer

- Dilatometry

- Diffraction

- Heat capacity measurements:

- drop calorimetry (DC),

$\circ$ differential scanning calorimetry (DSC)

- Thermal Conductivity

$\bigcirc \quad$ Laser flash method (thermal diffusivity)

- Transient hot wire method

Fortner et al. [66] identify standard procedures for specific experimental methods. For example, several operating procedures and test methods have been developed by ASTM International and other organizations:

- ASTM D2766 - 95 (2009). Standard Test Method for Specific Heat of Liquids and Solids.

- ASTM E1269-11. Standard Test Method for Determining Specific Heat Capacity by Differential Scanning Calorimetry.

- ASTM E967-08 (2014). Standard Test Method for Temperature Calibration of Differential Scanning Calorimeters and Differential Thermal Analyzers.

- ASTM E968-02. Standard Practice for Heat Flow Calibration of Differential Scanning Calorimeters.

- DIN 51007. General principles of differential thermal analysis.

- ASTM E1461 - 13 Standard Test Method for Thermal Diffusivity by the Flash Method

Similarly, Fortner et al. [66] identify several standard reference materials applicable to thermophysical property measurements:

- $\quad$ NIST SRM 720- Sapphire, heat capacity (10 K to $2250 \mathrm{~K})$.

- $\quad$ NIST SRM 781D2- Molybdenum, heat capacity (273.15 to $2800 \mathrm{~K}$ ).

- NIST SRM 2234- Gallium for thermal analysis (Melting temperature, enthalpy of fusion at $302.9146 \mathrm{~K})$.

- NIST SRM 2235- Bismuth for thermal analysis (Melting temperature, enthalpy of fusion at $544.556 \mathrm{~K})$.

- $\quad$ NIST SRM 1450d- Fibrous glass board $\left(280 \mathrm{~K}\right.$ to $\left.340 \mathrm{~K}, \sim 0.03 \mathrm{Wm}^{-1} \mathrm{~K}^{-1}\right)$

- NIST SRM 8420- Electrolytic iron $\left(2 \mathrm{~K}\right.$ to $\left.1000 \mathrm{~K}, \sim 30-100 \mathrm{Wm}^{-1} \mathrm{~K}^{-1}\right)$

Standard reference materials are lacking for some relatively new experimental methods, such as the laserflash thermal diffusivity technique. For these cases, well-characterized simple salts $(\mathrm{NaCl}, \mathrm{KCl})$ can be used as instrument checks before and following the measurements of salt samples to indicate proper operation. 
As discussed by Fortner et al. [66], one of the key issues with data quality for molten salt property measurements is salt purity and sample homogeneity. The ubiquitous presence of $\mathrm{O}_{2}$ and $\mathrm{H}_{2} \mathrm{O}$ as well as the possibility of significant levels of impurities (cations and anions) in commercially available pure component salts pose significant challenges. There is however, significant experience and expertise within the DOE National Laboratory complex in dealing with these challenges. Therefore, one of the key first steps in establishing rigorous standard practices for salt property measurements to be made as part of the MSR campaign will be establishing inter-laboratory protocols for the production and purification of salts to be tested. These protocols will include suggested sampling and subsampling techniques to ensure homogeneity. Particular areas that will be addressed in these protocols include:

- Best practices for maintaining an inert atmosphere environment when working with salt mixtures at elevated temperatures,

- Selection of a suitable molten salt container and crucible materials for elevated temperature work,

- Container/crucible materials that are compatible with the halide salt to be measured must be used. Microporous graphite should be "baked out" under vacuum to remove residual moisture/oxygen or glassy carbon used.

- Accurate, reliable determination of the salt composition,

○ Melting point check

○ Chemical analyses: X-ray fluorescence, mass spectrometry

As part of the MSR Campaign's efforts to establish the highest possible quality thermophysical and thermochemical molten salt databases, technical assessments similar to those in Fortner et al. [66] will be applied to all relevant salt compositions and property measurement techniques to identify critical aspects to be controlled to ensure quality data are generated. This effort will be performed as part of each activity conducted to determine salt property values and their dependencies.

\section{THERMOCHEMICAL MODELS AND DATABASE DEVELOPMENT}

\subsection{MODELS}

The recommended approach for representing the thermochemical behavior should rely on the CALPHAD method [67] to link solution phase models to their physical and chemical properties. The molar Gibbs energy of these solutions can be viewed as comprised of three parts given by the equation below.

$G=G^{r e f}-T \Delta S+G^{x s}$

Here the first two terms represent an ideal mixing. $G^{\text {ref }}$ is a weighted average of the Gibbs energies of each mixing constituent, the so called 'end members,' $T$ is temperature, $\Delta \mathrm{S}$ is the configurational entropy and $G^{x s}$ modifies ideal behavior by taking into account interactions. The convention is to use a polynomial expansion in $T$ and composition to represent $G^{x s}$, for example the Redlich Kister equation for binary solution given below.

$G^{x s}=x_{1} x_{2}{ }^{0} L+x_{1} x_{2} \sum{ }^{i} L\left(x_{1}-x_{2}\right)^{i}$

where $x_{i}$ are molar fractions of component $i$ and ${ }^{i} L$ are termed interaction parameters. 
Coefficients of $G^{x s}$ and, in some cases fictive or hypothetical Gibbs energy functions of the end members, are optimized using the largest reliable data set possible. This both improves confidence when interpolating within the limits of the experimental measurements and offers higher fidelity thermodynamic predictive capabilities.

The vapor phase at conditions relevant to MSRs are well within the $T$ - $P$ regime, where $P$ is pressure, to be treated as an ideal gas mixture. Therefore, the $G^{x s}$ term can be neglected. Stoichiometric compounds and pure substances are modeled as simple Gibbs energy polynomials with coefficients of temperature. The solution phase that needs the most attention for MSRs is, as one might expect, the molten salt. The modified quasichemical model (MQM) and the two-sublattice ionic liquid model (TSLM) are extensively used within the CALPHAD community to represent these melts. Of the two, the MQM incorporates more physics and better describes the configurational entropy by accounting for nearest neighbor and next nearest neighbor short range ordering. However, the TSLM is convenient to use and can do as well as the MQM in representing the Gibbs energy of both ionic and metallic melts, in the latter case it reduces to the regular solution model. Due to the existing body of literature reporting use of the MQM for molten salts [68-72] and the considerations discussed above, that model should be used to represent the liquid phase.

The CALPHAD solution models can be extended to represent the highly multicomponent systems that will inevitably evolve in the molten salt fuel during operation. Further, they can be coupled to other physics to predict transport phenomena, nucleation, precipitation, corrosion and other material performance behavior.

\subsection{APPLICATIONS}

\subsubsection{Corrosion}

Experience has indicated that corrosion of structural materials within a fluoride cooled or fueled MSR will occur, and that selection/development of materials that can sufficiently resist corrosive attack is a challenge. $[73,74]$ Current thinking is that nickel-based alloys similar to Hastelloy-N will be used, and thus systems largely containing nickel, molybdenum, and chromium. While molten salt corrosion of alloys are complex phenomena, an understanding of the thermochemical driving forces and stable phases/species can be helpful in characterizing and formulating rate-controlling mechanisms. Thus, the ability to model the equilibrium state of complex salts together with prospective structural alloys needs to be pursued. The effect of fluoride salt redox state on corrosion is recognized, and thus the ability to compute the redox state and identify transitions can be valuable. It has been observed for example, that in molten fluoride salt containing tellurium, the oxidation potential substantially influences the extent of cracking in Hastelloy-N.[75]

To illustrate the type of information that thermochemical models of fuel-alloy behavior provide, the limited database for $\mathrm{LiF}-\mathrm{UF}_{4}-\mathrm{UF}_{3}-\mathrm{BeF}_{2}$ (where $\mathrm{UF}_{3}$ is treated as having zero interaction energies with respect to other components) was used to determine fluorine potential reaction thresholds. Equilibrium states with nickel and chrome were computed as a function of fluorine activity at $700^{\circ} \mathrm{C}$ with the corresponding computed U(IV) to U(III) ratio, i.e., the redox potential (Fig. 1). Such calculations thus provide some insight into mechanisms and can indicate possible regions of concern. Other mechanisms are seen to cause possible cracking in oxidative regions, and thus it is expected that a balance between too reducing or too oxidative conditions will need to be maintained.[75] Thus operational guidance is provided from a combination of thermochemical simulations and observations of material behavior, 


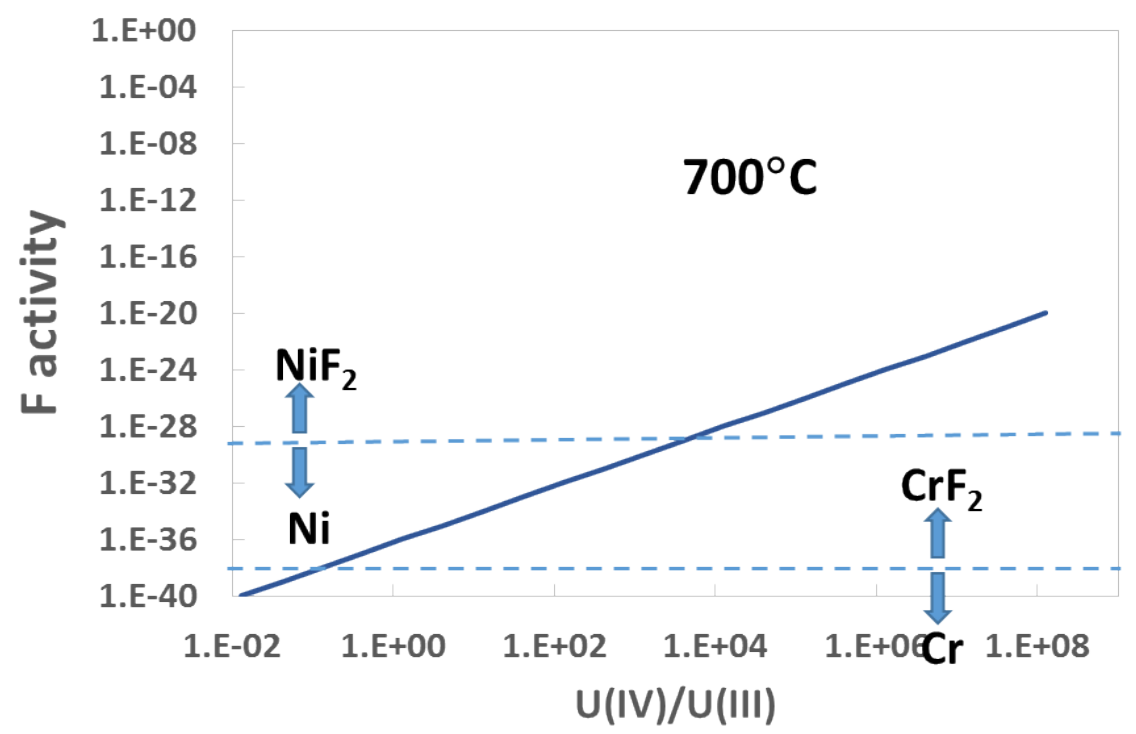

Figure 9 Computed fluorine activity versus redox (ratio of U(IV) to U(III)).

\subsubsection{Phase Equilibria}

A thermochemical database can be used to compute phase diagrams for binary and ternary systems of interest for MSRs. The real value of such a database for complex MSR fuel systems, however, is seen in representing many-element compositions. As liquid-fuel and/or simply molten salt coolant reside in the reactor, composition will vary with time. As noted earlier, transuranic formation, fission product generation, corrosion, and contamination will result in composition changes. It is worth pointing out that elements apart from those in the fresh salt will always be minor constituents. The ultimate goal for design and analysis purposes, and in real time for monitoring reactor/fuel performance, is the capability to supply the thermochemical state of the fuel. Again, for purposes of maintaining appropriate fluid state, for controlling precipitation/plating, to understand composition and therefore reactivity, and for redox control.

A means for visualizing such complex phase equilibria is via pseudo-binary or -ternary diagrams. Figure 9 and Figure 10 are examples of such diagrams. A pseudo-ternary liquidus projection with invariant points for $\mathrm{LiF}-\mathrm{UF}_{4}-\mathrm{ThF}_{4}$ computed from a proprietary database accompanying the FactSage software suite[76] is seen in Figure 10, where the computed states include the fixed fractions of $0.05 \mathrm{CrF}_{2}$ and 0.05 $\mathrm{UF}_{3}$. Similarly, Figure 11 is a diagram computed from the initial $\mathrm{LiF}_{-} \mathrm{BeF}_{2}-\mathrm{UF}_{4}$ database from the JRCKarlsruhe, now including $\mathrm{UF}_{3}$ at a fixed fraction of 0.05 (again, lacking interaction energy terms). Such diagrams allow manual exploration of composition-temperature space for multi-element systems, providing insight into the same calculations being performed during reactor/fuel simulations with dozens of elements varying with time and position in the reactor. 


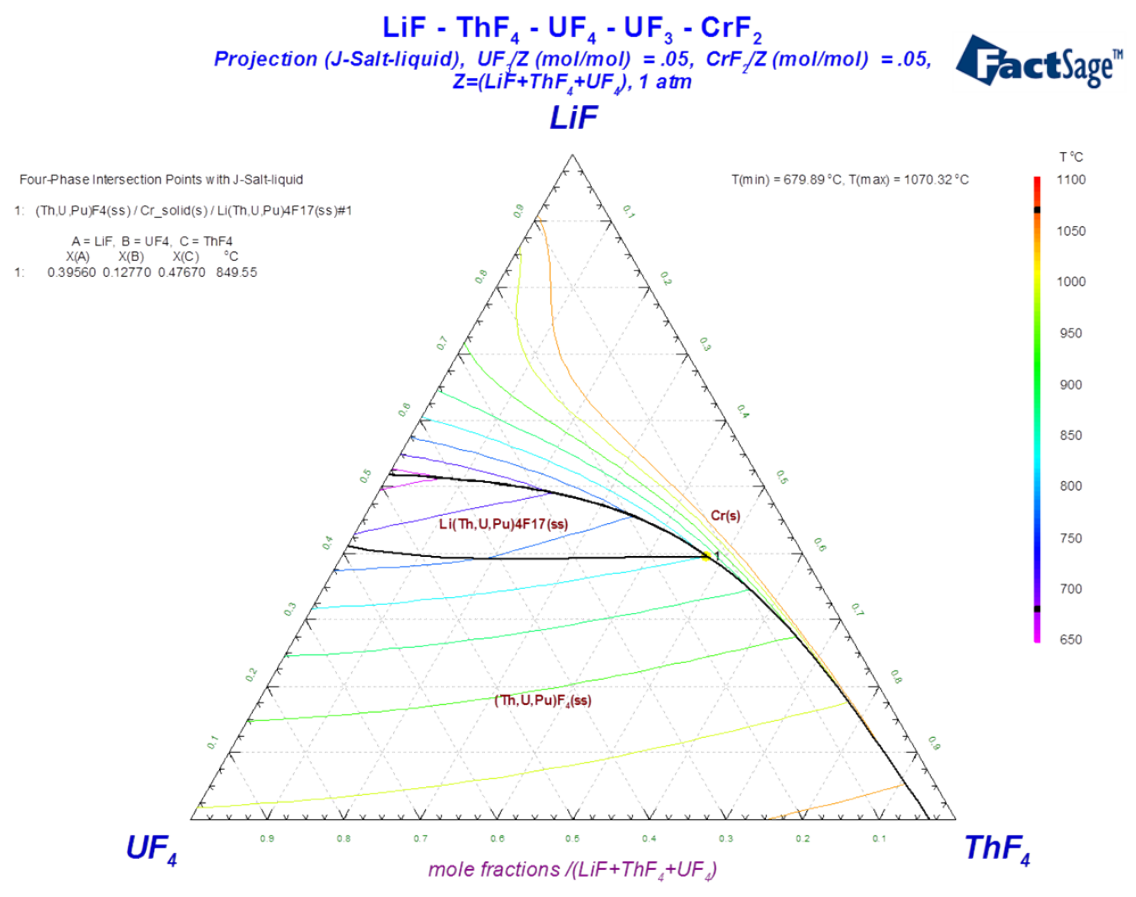

Figure 10 Liquidus projection and invariant points with phase boundaries for LiF-UF $-\mathrm{ThF}_{4}$ with fixed fractions of $0.05 \mathrm{CrF}_{2}$ and $0.05 \mathrm{UF}_{3}$. Computed using proprietary FactSage [77] database.

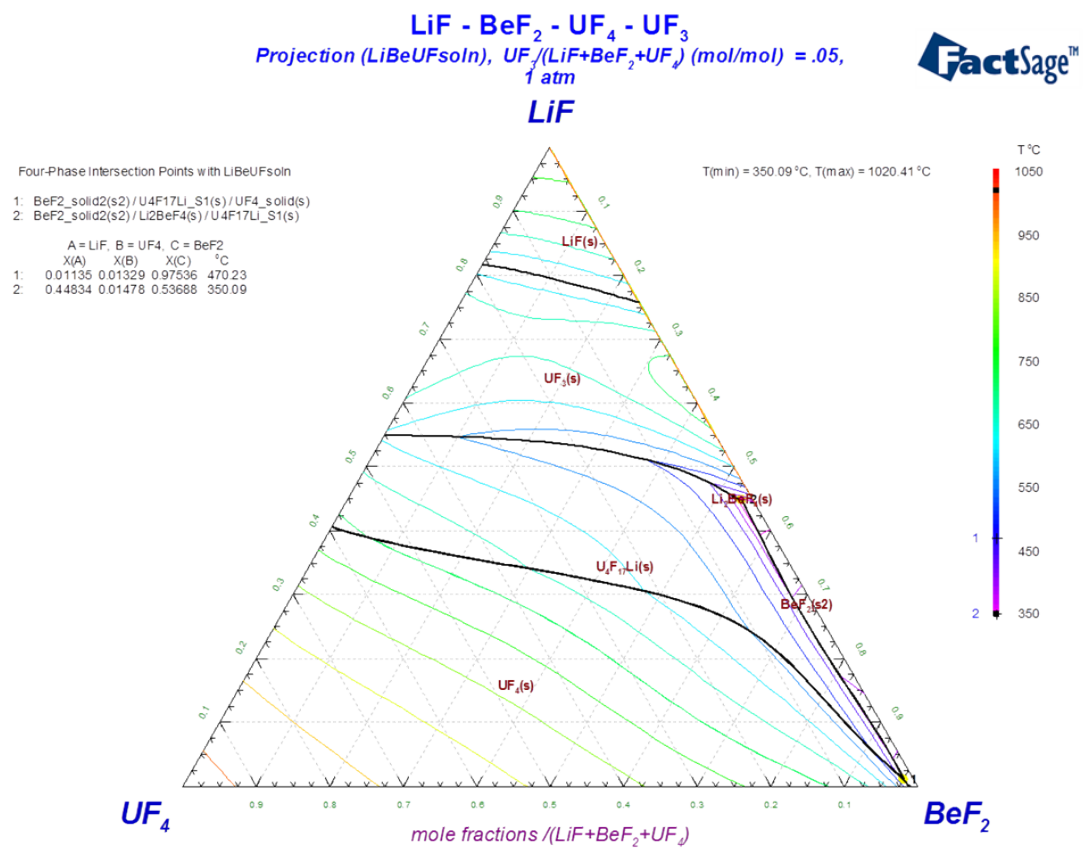

Figure 11 Liquidus projection and invariant points with phase boundaries for $\mathrm{LiF}-\mathrm{UF}_{4}-\mathrm{BeF}_{2}$ with fixed fraction of $0.05 \mathrm{UF}_{3}$. Computed using initial database from the JRC-Karlsruhe with added $\mathrm{UF}_{3}$ species. 


\subsubsection{Heat and Mass Transport}

A thermochemical database inherently contains the thermal properties of the constituents and allows direct computation of released or absorbed heats of reaction. The FactSage computational suite [76] provides thermal properties output from either directly written reactions or properties of material assemblages in their equilibrium state and the result of transitions to the equilibrium state. Below is an example output from FactSage for equilibrium calculations for a complex composition offering the product heat capacity, and in the case where reactions from non-standard states is considered, changes in enthalpy, entropy, etc. Note that values in the output are "absolute" values of enthalpy, entropy, and free energy, of which only that for entropy has physical significance. The heat capacity, however, is the heat capacity of the product, which in this case is single-phase molten salt of the composition below.

Input

$\begin{array}{ll}\mathrm{T}=700^{\circ} \mathrm{C} & \\ \mathrm{P}=1 \mathrm{~atm} & \\ & \\ \text { STREAM CONSTITUENTS } & \text { AMOUNT/mol } \\ \text { LiF } & 5.7300 \mathrm{E}-01 \\ \text { BeF2 } & 2.8700 \mathrm{E}-01 \\ \text { UF4 } & 1.4000 \mathrm{E}-01 \\ \text { UF3 } & 1.0000 \mathrm{E}-02\end{array}$

$\underline{\text { Output }}$

\begin{tabular}{|c|c|c|c|c|}
\hline $\mathrm{Cp}$ & $\mathrm{H}$ & S & G & V \\
\hline $\mathrm{J} . \mathrm{K}-1$ & $\mathrm{~J}$ & $\mathrm{~J} . \mathrm{K}-1$ & $\mathrm{~J}$ & dm3 \\
\hline $8747695+01$ & x*xᄎ & 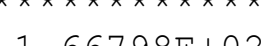 & 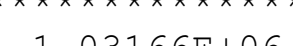 & 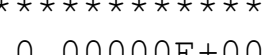 \\
\hline
\end{tabular}

Similar calculations can be performed, however by specifying non-standard conditions real changes in energy can be obtained in the transition from the starting conditions to final equilibrium conditions. For example, the salt constituents above can be assumed to originally be at room temperature, $25^{\circ} \mathrm{C}$, and then the salt is mixed and brought to $700^{\circ} \mathrm{C}$. The computed_values will reflect both the mixing and heating requirements, as seen below. The input amounts for all constituents are shown along with initial temperature, and the initial thermal properties provided, again absolute values for heat capacity, enthalpy, entropy, and free energy are provided with the same caveat as noted above.

$\underline{\text { Input }}$

$\mathrm{T}=700 \mathrm{C}$

$\mathrm{P}=1 \mathrm{~atm}$

$\mathrm{V}=0 \mathrm{dm} 3$

\begin{tabular}{|c|c|c|c|}
\hline STREAM CONSTITUENTS & AMOUNT/mol & TEMPERATURE/C & PRESSURE/atm \\
\hline LiF_Griceite_(NaCl_rock_ & $5.7300 \mathrm{E}-01$ & 25.00 & $1.0000 \mathrm{E}+00$ \\
\hline 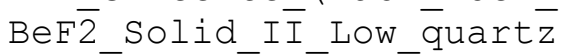 & $2.8700 \mathrm{E}-01$ & 25.00 & $1.0000 \mathrm{E}+00$ \\
\hline UF4_solid - - - & $1.4000 \mathrm{E}-01$ & 25.00 & $1.0000 \mathrm{E}+00$ \\
\hline UF3_solid & $1.0000 \mathrm{E}-02$ & 25.00 & $1.0000 \mathrm{E}+00$ \\
\hline 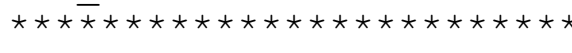 & 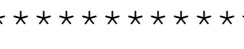 & 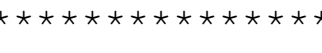 & 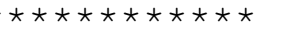 \\
\hline $\begin{array}{l}\mathrm{CP} \_ \text {INI } \\
\mathrm{J} \cdot \mathrm{K}-1\end{array}$ & $\begin{array}{l}S \_I N I \\
J \cdot K-1\end{array}$ & $G_{-}^{G} I N I$ & $\begin{array}{c}V_{\overline{d m} 3} \text { INI } \\
\text { dm }\end{array}$ \\
\hline
\end{tabular}




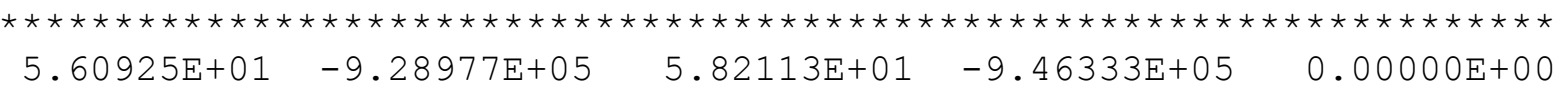

The results of the calculations are shown below, again for the product single-phase molten salt. The first set of values prefaced with "DELTA" represent the change in state from the separate input materials at $25^{\circ} \mathrm{C}$ to the molten salt mixture at $700^{\circ} \mathrm{C}$. The following set of values represent the product properties, with the heat capacity and entropy only having direct physical meaning. All such results can be obtained from any general thermodynamic equilibrium solver, and thus can be extracted from the simulations performed by Thermochimica, for example, operating as coupled in MSR reactor/fuel performance codes.

Output

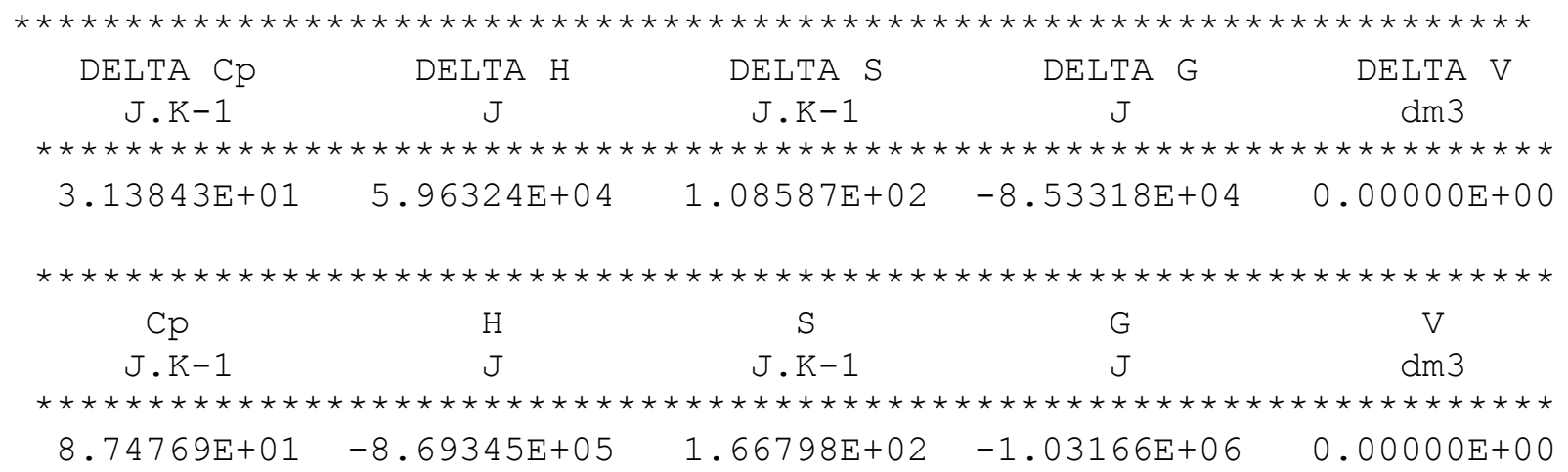

Non-equilibrium results can be obtained provided knowledge of the non- or sluggishly-forming phases is available and thus they can be omitted from the calculation of the equilibrium state. Reacting flow can be simulated via "open" calculations which allow small aliquots of the total fluid volume to react with subsequent removal of the products and appropriate adjustment of the remaining reactant composition. Such capability is currently in FactSage and similar proprietary software, and relatively easily generated in open source codes such as Thermochimica,[78] recommended for use within MSR reactor/fuel performance codes.

\subsubsection{Source Term Tracking}

The molten salt equilibrium state directly provides chemical activities for all constituents, which include fugacity (activity) for all gaseous/vapor species. Thus, the partial pressure for species are known and can be used to determine, at least under equilibrium conditions, the vapor pressure of active species to provide source terms for accident analysis. These types of calculations have been actively used in determining possible vapor pressures of radionuclides under accident conditions, providing initial information for subsequent detailed analysis of the kinetics and mass transport behavior. Thus, they form the basis for determining source terms, and for MSRs where an aqueous environment is avoided, can even more directly provide transport rates of radionuclide species. While there are currently insufficient thermodynamic models for source term elements of interest in fluoride-based fuel systems, (e.g., cesium, iodine, and strontium), one can look to analogous efforts in oxide fuels to note what can be determined. Examples are seen in Figure 12 and Figure 13 which are computed equilibrium state vapor pressures for some LWR fuel volatile, reactive species that were used in the further development of the MELCOR code.[79] 


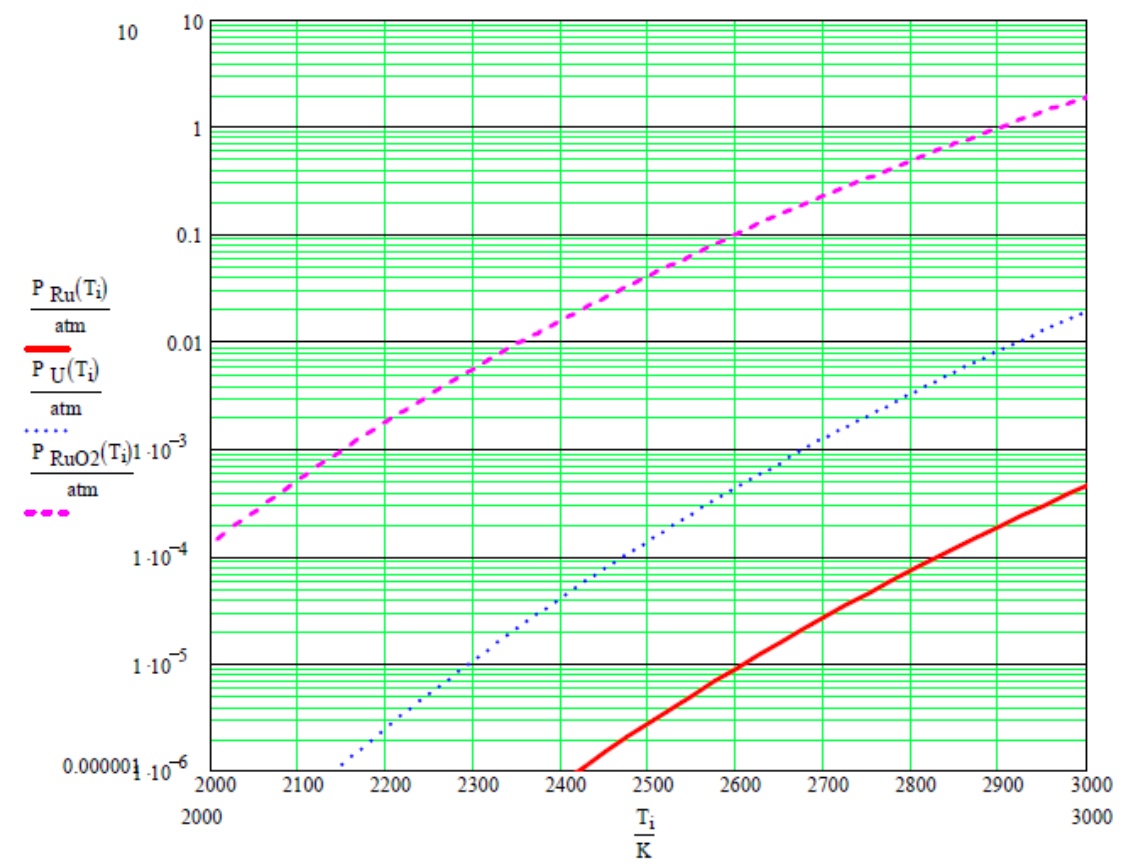

Figure 12 Ruthenium species vapor pressures computed from equilibrium state of LWR fuel utilized in MELCOR.[79]

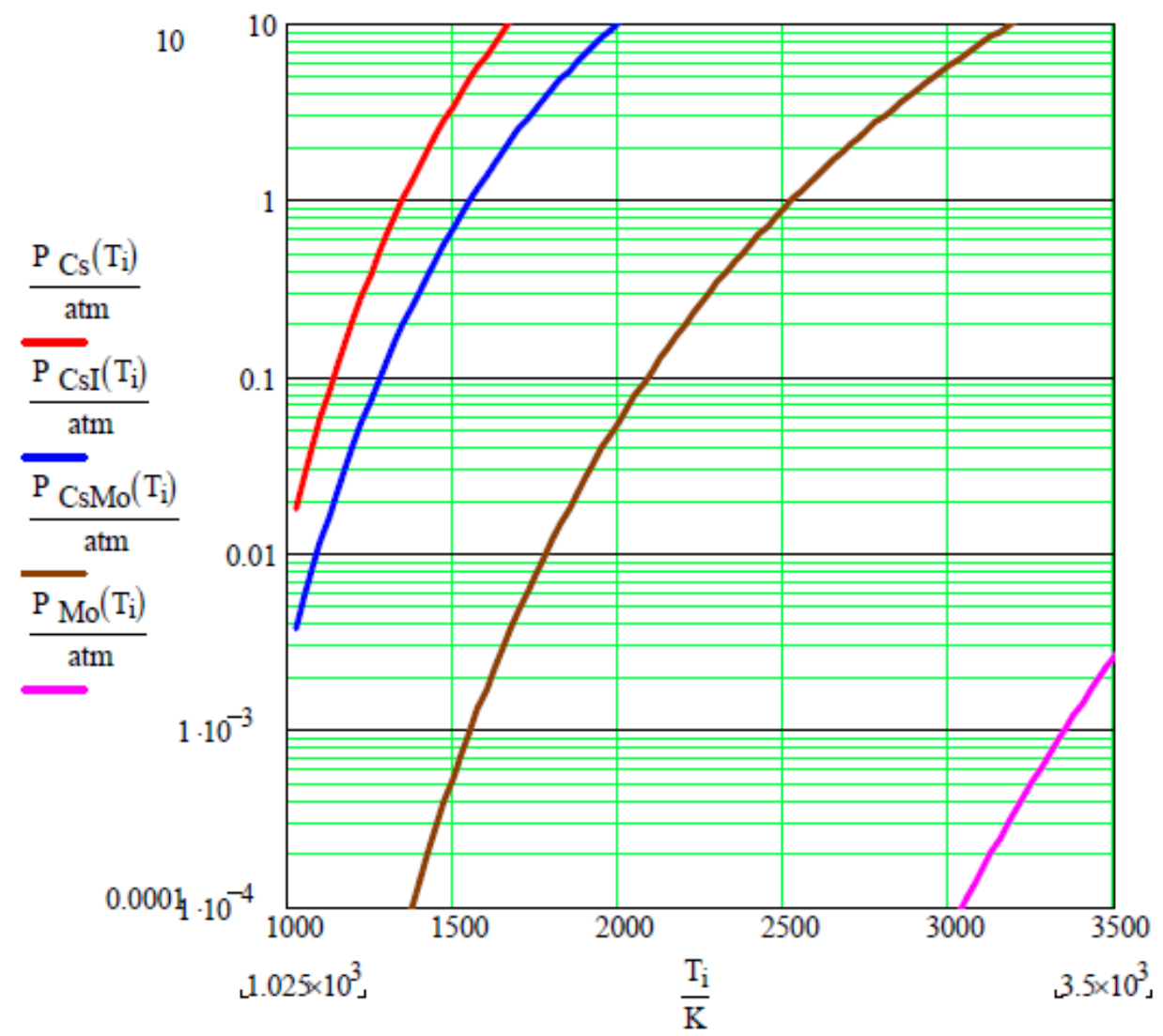

Figure 13 Cesium species vapor pressures computed from equilibrium state of LWR fuel utilized in MELCOR.[79] 


\subsection{GAP ANALYSIS}

\subsubsection{Fluoride Systems}

Three fluoride-based US MSR concepts and their corresponding compositions are shown in Table 13. A great deal of research has been performed for these compositions, and thermodynamic models are available for several of the subsystems. Figure 14 and Figure 15 show the current state of binary and ternary systems which have published models. Most of these modeled systems include consideration of any solid solutions in addition to the molten salt liquid solution.

Table 13 Main fluoride-based US MSR concepts [22, 80].

\begin{tabular}{|c|c|c|c|}
\hline Reactor Design & Neutron Spectrum & Primary Composition & $\begin{array}{l}\text { Additional Compounds of } \\
\text { Interest }\end{array}$ \\
\hline \multirow[t]{2}{*}{ Breeder } & Thermal & ${ }^{7} \mathrm{LiF}-\mathrm{BeF}_{2}-\mathrm{ThF}_{4}-\mathrm{PuF}_{3}-\mathrm{UF}_{4}$ & $\mathrm{ZrF}_{4}, \mathrm{PuF}_{4}, \mathrm{UF}_{3}$ \\
\hline & Fast & ${ }^{7} \mathrm{LiF}-\mathrm{ThF}_{4}-\mathrm{PuF}_{3}-\mathrm{UF}_{4}$ & $\mathrm{NaF}, \mathrm{BeF}_{2}, \mathrm{CaF}_{2}, \mathrm{PuF}_{4}, \mathrm{UF}_{3}$ \\
\hline Actinide Burner & Fast & LiF-NaF-BeF $2-\mathrm{PuF}_{3}-\mathrm{UF}_{4}$ & $\mathrm{KF}, \mathrm{RbF}, \mathrm{PuF}_{4}, \mathrm{UF}_{3}$ \\
\hline
\end{tabular}

\begin{tabular}{|l|c|c|c|c|c|c|c|c|c|c|}
\hline & $\mathrm{NaF}$ & $\mathrm{BeF}_{2}$ & $\mathrm{KF}$ & $\mathrm{RbF}$ & $\mathrm{CaF}_{2}$ & $\mathrm{ZrF}_{4}$ & $\mathrm{ThF}_{4}$ & $\mathrm{PuF}_{3}$ & $\mathrm{UF}_{4}$ & $\mathrm{UF}_{3}$ \\
\hline $\mathrm{LiF}$ & $\checkmark$ & $\checkmark$ & $\checkmark$ & $\checkmark$ & $\checkmark$ & $\checkmark$ & $\checkmark$ & $\checkmark$ & $\checkmark$ & $\checkmark$ \\
\hline $\mathrm{NaF}$ & & $\checkmark$ & $\checkmark$ & $\checkmark$ & $\checkmark$ & & $\checkmark$ & $\checkmark$ & $\checkmark$ & $\checkmark$ \\
\hline $\mathrm{BeF}_{2}$ & & & & & & $\checkmark$ & $\checkmark$ & $\checkmark$ & $\checkmark$ & \\
\hline $\mathrm{KF}$ & & & & $\checkmark$ & $\checkmark$ & & & $\checkmark$ & & \\
\hline $\mathrm{RbF}$ & & & & & & & & $\checkmark$ & & \\
\hline $\mathrm{CaF}_{2}$ & & & & & & & $\checkmark$ & & & \\
\hline $\mathrm{ZrF}_{4}$ & & & & & & & & & $\checkmark$ & \\
\hline $\mathrm{ThF}_{4}$ & & & & & & & & $\checkmark$ & $\checkmark$ & \\
\hline $\mathrm{PuF}_{3}$ & & & & & & & & & $\checkmark$ & \\
\hline $\mathrm{UF}_{4}$ & & & & & & & & & & $\checkmark$ \\
\hline
\end{tabular}

Figure 14 Binary subsystems of LiF-BeF $2-\mathrm{NaF}_{2} \mathrm{ThF}_{4}-\mathrm{PuF}_{3}-\mathrm{UF}_{4}-\mathrm{UF}_{3}-\mathrm{ZrF}_{4}-\mathrm{KF}-\mathrm{RbF}-\mathrm{CaF}{ }_{2}$ 


\begin{tabular}{|c|c|c|c|c|c|c|c|c|c|c|c|}
\hline & $\mathrm{LiF}$ & $\mathrm{BeF}_{2}$ & $\mathrm{NaF}$ & $\mathrm{ThF}_{4}$ & $\mathrm{PuF}_{3}$ & $\mathrm{UF}_{4}$ & $\mathrm{UF}_{3}$ & $\mathrm{ZrF}_{4}$ & $\mathrm{KF}$ & $\mathrm{RbF}$ & $\mathrm{CaF}_{2}$ \\
\hline $\mathrm{LiF}-\mathrm{BeF}_{2}$ & & & $\checkmark$ & $\checkmark$ & $\checkmark$ & $\checkmark$ & & $\checkmark$ & & & \\
\hline LiF-NaF & & & & $\checkmark$ & $\checkmark$ & $\checkmark$ & & & $\checkmark$ & & $\checkmark$ \\
\hline $\mathrm{BeF}_{2}-\mathrm{NaF}$ & & & & $\checkmark$ & $\checkmark$ & $\checkmark$ & & & & & \\
\hline $\mathrm{LiF}-\mathrm{ThF}_{4}$ & & & & & $\checkmark$ & $\checkmark$ & & & & & \\
\hline $\mathrm{BeF}_{2}-\mathrm{ThF}_{4}$ & & & & & & $\checkmark$ & & & & & \\
\hline $\mathrm{NaF}_{-} \mathrm{ThF}_{4}$ & & & & & & $\checkmark$ & & & & & \\
\hline LiF-PuF 3 & & & & & & $\checkmark$ & & & & & \\
\hline \multicolumn{12}{|l|}{$\mathrm{BeF}_{2}-\mathrm{PuF}_{3}$} \\
\hline \multicolumn{12}{|l|}{$\mathrm{NaF}-\mathrm{PuF} \mathrm{F}_{3}$} \\
\hline \multicolumn{12}{|l|}{$\mathrm{ThF}_{4}-\mathrm{PuF}_{3}$} \\
\hline $\mathrm{LiF}_{-\mathrm{UF}_{4}}$ & & & & & & & & $\checkmark$ & & & \\
\hline $\mathrm{BeF}_{2}-\mathrm{UF}_{4}$ & & & & & & & & $\checkmark$ & & & \\
\hline \multicolumn{12}{|l|}{$\mathrm{NaF}_{-\mathrm{UF}_{4}}$} \\
\hline \multicolumn{12}{|l|}{$\mathrm{ThF}_{4}-\mathrm{UF}_{4}$} \\
\hline \multicolumn{12}{|l|}{$\mathrm{PuF}_{3}-\mathrm{UF}_{4}$} \\
\hline \multicolumn{12}{|l|}{$\mathrm{ZrF}_{4}-\mathrm{UF}_{3}$} \\
\hline \multicolumn{12}{|l|}{$\mathrm{KF}_{-} \mathrm{UF}_{3}$} \\
\hline \multicolumn{12}{|l|}{$\mathrm{RbF}_{-} \mathrm{UF}_{3}$} \\
\hline \multicolumn{12}{|l|}{$\mathrm{CaF}_{2}-\mathrm{UF}_{3}$} \\
\hline \multicolumn{12}{|l|}{$\mathrm{KF}-\mathrm{ZrF}_{4}$} \\
\hline \multicolumn{12}{|l|}{$\mathrm{RbF}-\mathrm{ZrF}_{4}$} \\
\hline \multicolumn{12}{|l|}{$\mathrm{CaF}_{2}-\mathrm{ZrF}_{4}$} \\
\hline $\mathrm{KF}-\mathrm{RbF}$ & $\checkmark$ & $\checkmark$ & & & & & & & & & \\
\hline $\mathrm{KF}-\mathrm{CaF}_{2}$ & $\checkmark$ & $\checkmark$ & & & & & & & & & \\
\hline $\mathrm{RbF}-\mathrm{CaF}_{2}$ & & & & & & & & & & & \\
\hline
\end{tabular}

Figure 15 Ternary subsystems of $\mathrm{LiF}-\mathrm{BeF}_{2}-\mathrm{NaF}-\mathrm{ThF}_{4}-\mathrm{PuF}_{3}-\mathrm{UF}_{4}-\mathrm{UF}_{3}-\mathrm{ZrF}_{4}-\mathrm{KF}-\mathrm{RbF}-\mathrm{CaF}_{2}$

In addition to the main components of the compositions, it is necessary to investigate the interaction of dilute constituents which may enter the MSR system during operation, or as initial contaminants. This includes fission products, transuranics, corrosion products, and contaminants such as oxygen and nitrogen. These dilute constituents need only be investigated with regard to their interactions with the main salt components, as they will be present in relatively small amounts. A few interactions between dilute constituents were found in literature. They are:

- $\mathrm{Ce}$ assessed in the $\mathrm{LiF}_{-} \mathrm{ThF}_{4}-\mathrm{CeF}_{3}$ and $\mathrm{LiF}-\mathrm{NaF}-\mathrm{CeF}_{3}$ systems.[81, 82]

- Am assessed as $\mathrm{AmF}_{3}$ in the LiF-NaF-KF-PuF $-\mathrm{UF}_{4}$ system. [83]

- $\mathrm{Cs}$ and I assessed in the $\mathrm{LiF}_{-} \mathrm{ThF}_{4}-\mathrm{CsF}-\mathrm{LiI}-\mathrm{ThI}_{4}-\mathrm{CsI}, \mathrm{CsF}-\mathrm{PuF}$, and $\mathrm{CsF}-\mathrm{LaF}_{3}$ systems.[84-86]

- $\mathrm{La}$ assessed in the LiF-NaF-RbF-LaF, $\mathrm{LaF}_{3}-\mathrm{PuF}_{3}$, and LiF-NaF-CaF $-\mathrm{LaF}_{3}$ systems. ${ }^{[85-87]}$

- Corrosion of $\mathrm{Ni}$ assessed in the $\mathrm{LiF}-\mathrm{NiF}_{2}, \mathrm{NaF}_{-} \mathrm{NiF}_{2}$, and $\mathrm{KF}-\mathrm{NiF}_{2}$ systems. ${ }^{[8]}$ 
- Corrosion of $\mathrm{Cr}$ interaction with $\mathrm{UF}_{3} / \mathrm{UF}_{4}$ by the reaction: $\mathrm{Cr}$ (alloy) $+2 \mathrm{UF}_{4} \rightarrow \mathrm{CrF}_{2}+$ $2 \mathrm{UF}_{3} \cdot[88]$

- $\quad \mathrm{Nb}-\mathrm{F}$ binary system assessed.[89]

- Both $\mathrm{O}_{2}$ and $\mathrm{N}_{2}$ have the potential to form numerous compounds with the main salt components.[90]

- $\quad \mathrm{Np}$ may form $\mathrm{NpF}_{\mathrm{x}}, \mathrm{Nd}$ may form $\mathrm{NdF}_{3}$, Pr may form $\operatorname{Pr} \mathrm{F}_{\mathrm{x}}$, Mo may form $\mathrm{MoF}_{\mathrm{x}}, \mathrm{Rh}$ may form $\mathrm{URh}_{3}$, Ru may form $\mathrm{Ru}_{\mathrm{x}} \mathrm{Zr}$ or $\mathrm{RuF}_{5}$, $\mathrm{Pd}$ may form $\mathrm{PdF}_{\mathrm{x}}$.

- No data for Tc, Sm, or Cm interacting with the main salt components.

\subsubsection{Chloride Systems}

A thermodynamic evaluation and optimization of chlorides of $\mathrm{Li}, \mathrm{Na}, \mathrm{K}, \mathrm{Rb}, \mathrm{Cs}, \mathrm{Mg}$, and $\mathrm{Ca}$ has been performed by Chartrand and Pelton [91]. They use assessed fundamental binary and ternary subsystems to develop MQM for multicomponent molten salts. While this approach is common and can produce good results, they are extrapolations into uncharted composition space. As was done for the fluorides, there is therefore a crucial need for thermochemical measurements to define and validate the multicomponent system behavior, including $U$, the actinides and fission products. The following methods are indicated: differential scanning calorimetry (DSC), thermogravimetry (TG), mass spectrometry and other evolved gas analysis techniques (EGA), and advanced characterization including but not limited to high temperature x-ray and neutron diffraction. Much more experimental and computational work is needed for the chloride systems compared to the fluorides. The paucity of data and literature therefore does not permit an in-depth analysis such as was presented above in 3.3.1.

In order to advance the thermodynamic description of these molten salt systems, there is still much work to be done for both the experimental and modeling efforts. Below are some of the major pieces still required to create a complete model listed in order of priority:

1. Obtain targeted experimental data focused towards the optimization of the interactions of the missing binary and ternary subsystems of highest relative composition.

2. Alongside the binary optimizations from item 1 , the cation-cation coordination numbers need also be determined for the subsystems that have yet to be modelled.

3. Determine the subsystems in need of a solid solution description, design experiments if needed, and create the solid solutions.

4. Design experiments for the dilute constituents which have yet to be investigated in literature and add to the model.

5. Determine if the optimization of any quaternary subsystems is necessary for the complete description, and if so, perform them.

6. Continually verify the model against experimental data and update as necessary as new data is discovered.

\section{COUPLING CHEMISTRY TO REACTOR PERFORMANCE MOD-SIM}

\subsection{THERMODYNAMIC SOLVER CHARACTERISTICS}

Thermochimica is a stand-alone thermodynamics solver that has been designed for direct integration into multi-physics codes [92]. Conventional CT software are generally used to perform a small handful of point calculations or to compute phase diagrams. Coupling to multi-physics codes places significantly greater requirements on computational performance due to the exceedingly large number of evaluations. As a result, a significant advantage to using Thermochimica is that during development great care has 
taken to implement effective numerical approaches to solve thermochemical equilibria. In particular, significant efforts have been placed on effective global optimization algorithms to handle non-convexity in the objective function [93].

Thermochimica requires as input temperature (double scalar), pressure (double scalar), mass of each chemical element (double vector), character variables for each of the aforementioned variables representing the units (e.g., 'kelvin'), and finally a character variable representing the data-file pathname. More details on usage can be found in the Thermochimica user manual [94]. Input and output operations associated with Thermochimica are best understood with respect to Figure 16.

On output, Thermochimica is able provide the following variables:

- Identification of stable phases and their quantities (i.e., moles).

- Speciation of solutions phases (i.e., mole fractions of species and site fractions of constituents).

- Integral Gibbs energy of system.

- Molar Gibbs energy of stable phases.

- Driving force of metastable phases.

- Chemical potentials of all chemical elements.

The following quantities can be provided with some additional programming effort:

- Heat capacity

- Enthalpy

- Entropy

- Partial thermodynamic properties (e.g., partial molar excess Gibbs energy of mixing of a species). 


\section{Input}

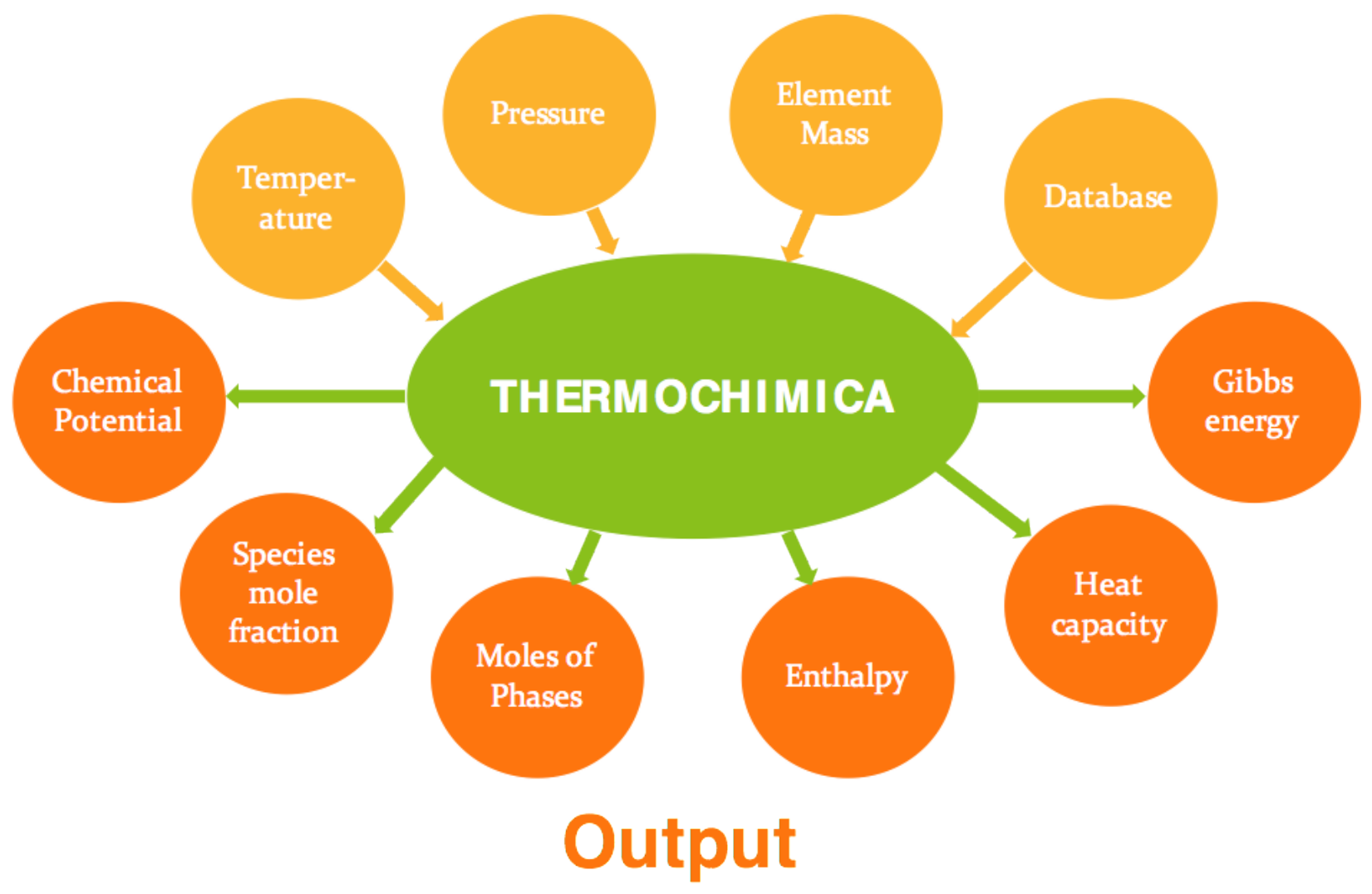

Figure 16 A schematic of input/output operations with Thermochimica.

Many of the aforementioned quantities are useful to other phenomena captured by the overall multiphysics framework. For instance, heat capacity and enthalpy are useful for heat transfer calculations, speciation of the salt can be used to compute viscosity for momentum equations, and phase quantities can be used for multi-phase flow calculations (e.g., combinations of salt, gas, and solid precipitates).

The next step in the development of Thermochimica is the implementation of the MQM model, which is indicated for representing molten salts and is described in greater detail by Pelton et al [68]. The MQM model is fundamentally different than other thermodynamic models in that the focus is not on the mixing of chemical species or constituents on a lattice, but rather the mixing of species as pairs to capture shortrange order in liquid or solid solutions.

Significant progress has been made in upgrading the capabilities of Thermochimica to handle the MQM model with various salts as a testbed, which is described in another report [95]. However, it is worth noting that a critical component to any such thermochemical calculations rests on having access to a suitable thermochemical database. 


\subsection{THERMOCHIMICA DEVELOPMENT AND INTEGRATION WITH ORIGEN AND COBRA-TF}

Thermochimica [96] is proposed for the thermodynamics solving capability. It is an equilibrium thermodynamics solver, specifically designed for use with multi-physics codes. Thermochimica works by finding unique combination of species and phases which minimizes the value of the integral Gibbs energy of an isothermal-isobaric closed system, under the constraints of the Gibbs phase rule and mass balance. Thermochimica uses many different thermodynamic models, and its capabilities are expanding to be suitable for molten salt reactors.

While thermodynamic equilibrium is not instantly attained in nuclear fuel under irradiation, the assumption of rapid equilibrium is sound [92]. Firstly, due to the high temperature of the fuel, chemical equilibrium is attained in comparatively short durations, whereas, due to fissioning, the chemical elements in the fuel are randomly mixed. In a molten salt reactor, where fission product atoms are in a liquid state, they are even more mobile, making this argument even more valid. Further, the timescales in nuclear performance simulations are typically very long. It is also clear that a significant fraction of the experimental efforts on nuclear material behavior have been centered on equilibrium conditions rather than time-dependent conditions.

As an example of the soundness of the rapid equilibrium assumption, consider a study comparing the phase distribution of irradiated solid $\mathrm{UO}_{2}$ in simulations with experiments on actual irradiated fuel [92]. In this work, Thermochimica was coupled with ORIGEN-S and Advanced Multi-Physics (AMP). It was found that, within experimental error, the phase distribution across the radius of a fuel pellet matched experimental results.

Work at the University of Ontario Institute of Technology (UOIT) is currently focused on incorporating the MQM into Thermochimica as well as interfacing it with ORIGEN [97] (isotope physics code) and COBRA-TF [98] (thermal-hydraulics code) using a $\mathrm{C}++$ framework. The interfacing contribution is illustrated in Figure 17.

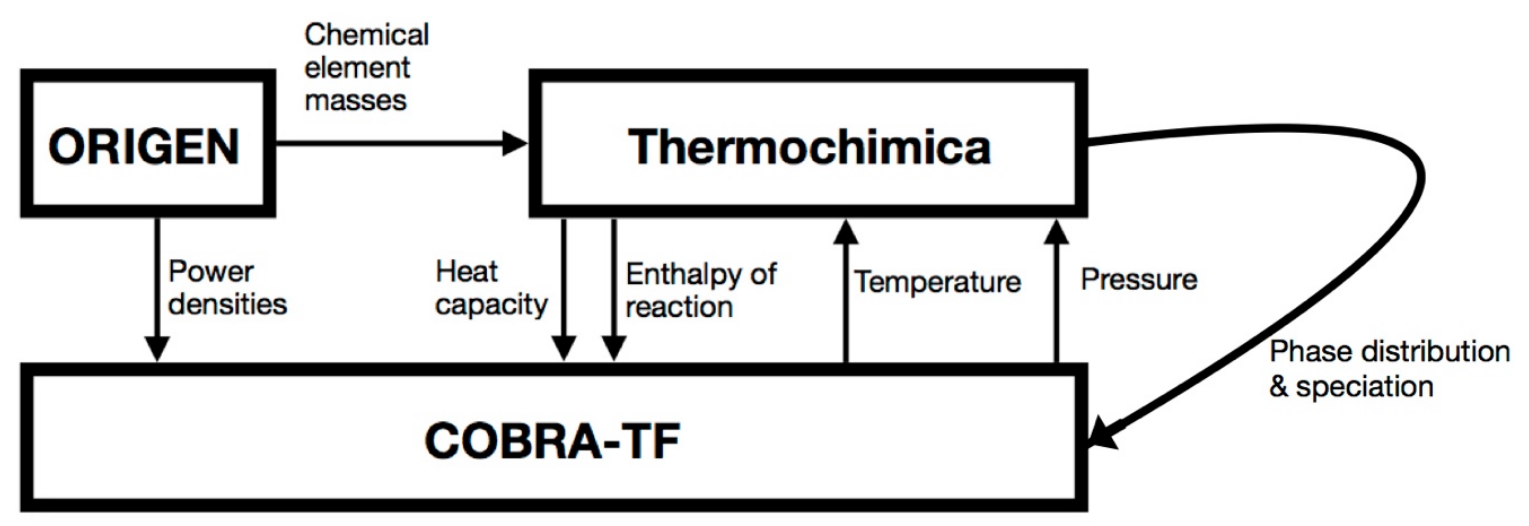

Figure 17 Interfacing contribution of UOIT, between Thermochimica (thermodynamics), ORIGEN (isotope physics) and COBRA-TF (thermal-hydraulics).

Integration of Thermochimica with ORIGEN for isotope physics via the ORIGEN API (application programming interface) has already been achieved. Consider a fictive demonstration in Figure 18 (isotopic evolution) and Figure 19 (phase evolution). Since materials libraries are not yet available for 
molten salt reactors, a demonstration simulation considers a system of 3.6\% enriched $\mathrm{UO}_{2}$ fuel [99]. However, a power history from the MSRE (Molten Salt Reactor Experiment) is employed [100]. The system studied is the noble metal system (Mo-Ru-Tc-Rh-Pd). Although they are not intended to be physical, these fictive simulations demonstrate the capability of the software and interfacing at this stage of development.

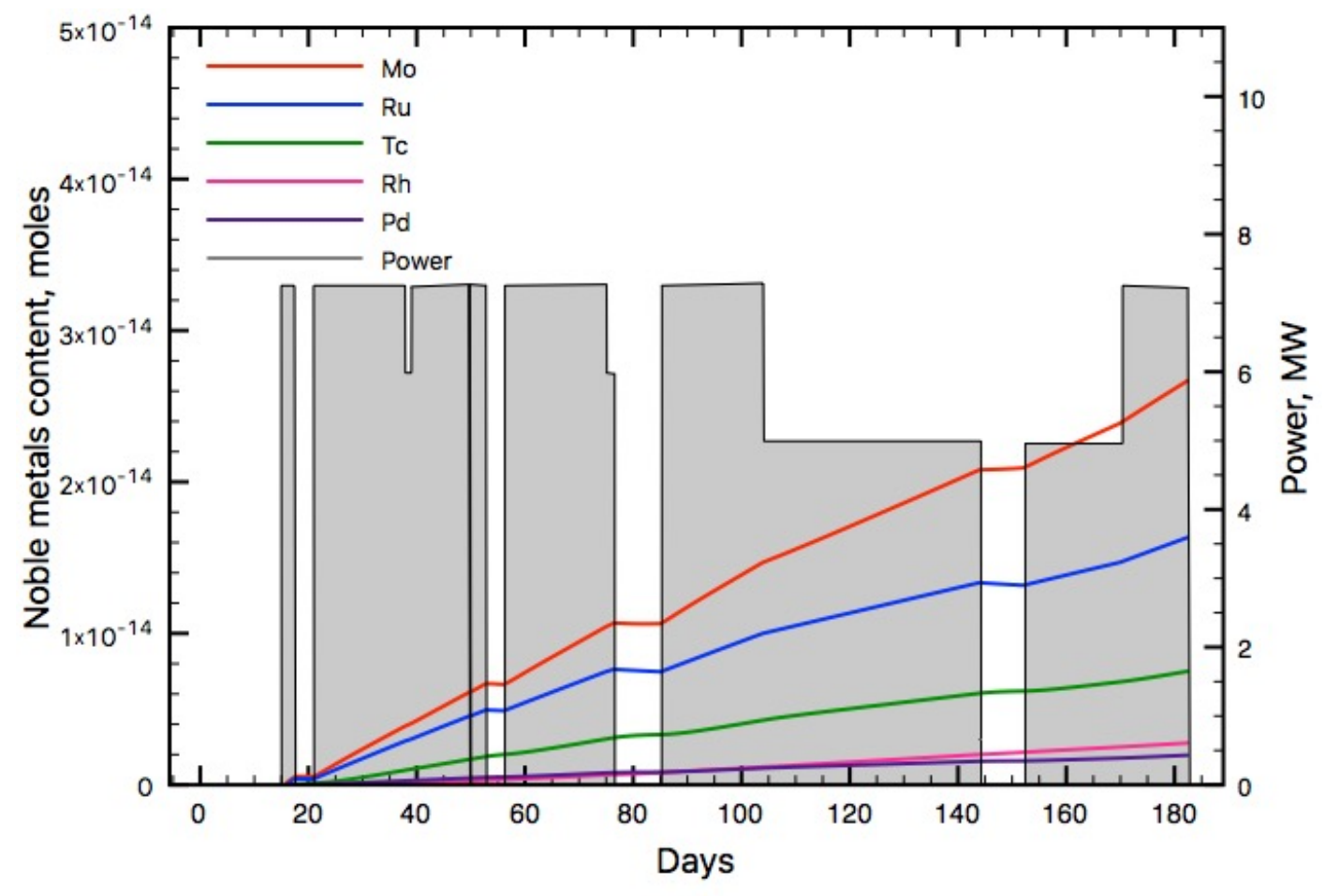

Figure 18 Isotopic evolution of the noble metal system, from ORIGEN, using a power history from the MSRE. Power history taken from [100]. 


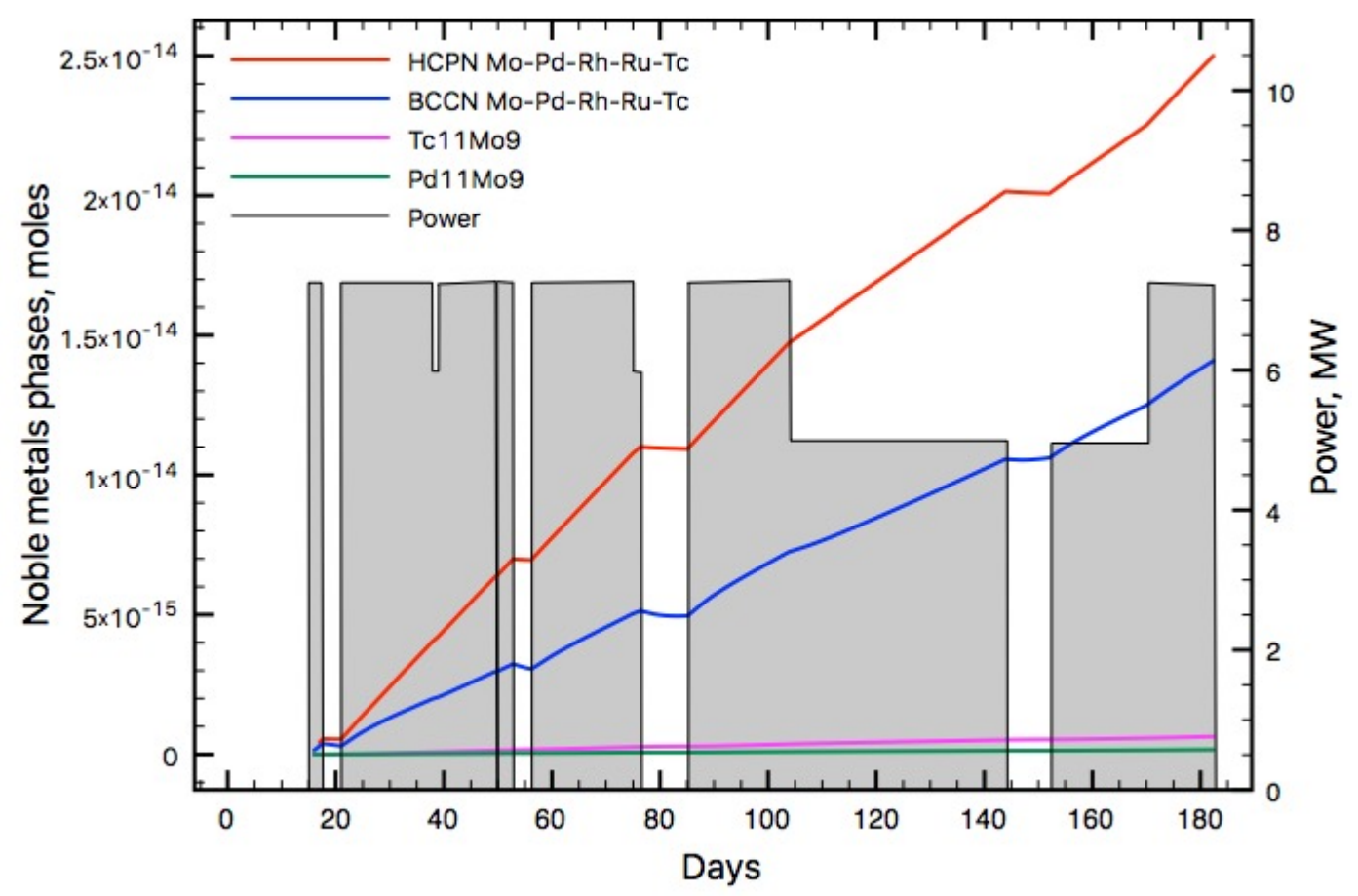

Figure 19 Thermodynamic simulation of phase evolution, from Thermochimica, coupled to the ORIGEN-API. Power history from [100].

In the above example, the interface considers a $60 \mathrm{~g}$ specimen of $3.6 \%$ enriched $\mathrm{UO}_{2}$ fuel. This is created as a "material" object using the ORIGEN API. A depletion calculation is performed in ORIGEN, and the isotopic distribution along 200 time points is transferred to an array. A simple algorithm then distills the distribution of over 2000 isotopes into a distribution of over 40 chemical elements, and then passes this information as a vector to Thermochimica. Thermochimica is called one time for every time point while assuming a constant temperature and pressure. More realistic physics will require interfacing with a thermal-hydraulic code.

\subsection{THERMOPHYSICAL DATA INTEGRATION INTO MOD-SIM FRAMEWORK}

Thermophysical data are material properties that impact heat storage and transfer (i.e., thermal hydraulics). This includes viscosity, thermal conductivity, heat capacity, thermal diffusivity, thermal expansion properties, mass diffusion coefficients, thermal radiative properties, and thermal diffusion coefficients. These properties are dependent on the chemical makeup of the material, as well as temperature, pressure, etc. For light water reactors, thermophysical data for the fuel pellet, gap, cladding, and water coolant are needed. This fuel pellet to water coolant heat transfer problem changes with fuel depletion as the pellet physically deforms under irradiation, the gap composition changes as it closes, and cladding elongates. As the gap composition changes, its thermophysical properties change. Under irradiation, the fuel pellet undergoes relocation due to cracking, densification from porosity reduction, and swelling due to fission product generation. Fuel performance tools model these changes and their impact on thermophysical properties of the fuel pellet (Stimpson et al., 2018), and thus thermal hydraulic calculations. Similar fuel performance tools are applicable for TRISO-based fuel forms.

In liquid-fueled molten salt reactors, structural and moderator materials may undergo some changes that effect their thermophysical properties (e.g., graphite). But, there are no concerns of cracking, 
densification, or swelling in a liquid fuel salt. It is the continuously changing chemical composition that drives the change in the thermophysical properties of the liquid fuel salt. While the rate of this change is dependent on reactor design and salt selection and may be gradual, high burnup objectives of many liquid-fueled designs increase the importance of knowing the thermophysical properties of a fuel salt that contains significant concentrations of fission products and actinides.

Following the measurement and uncertainty quantification for these thermophysical properties, the efficient storage and recall of quantities at a specific condition (e.g., temperatures and pressures) is important for high-fidelity modeling and simulation tools. These tools may need to recall hundreds or thousands of quantities on a very short time scale. Parallels do exist in the use of nuclear data in neutron transport: cross sections are tabulated in temperature and energy, and Monte Carlo transport tools first generate a set to use at a given temperature and during the transport repeatedly searches the list and interpolates to generate a cross section at a given energy. With this parallel, it is reasonable to imagine a precomputed dataset of thermophysical properties (with uncertainties) tabulated in relevant condition (e.g., temperature) for a given chemical composition (e.g., fresh FLiBe). Multiple datasets with different chemical compositions may then be generated to effectively tabulate a given initial composition in burnup, impurity, for fission product concentration.

\subsection{DATABASE REQUIREMENTS}

The highest level functional requirements for the molten salt chemistry database include the following:

1. The data will be stored on secure computer systems controlled by a national laboratory using accepted protocols.

2. The data will be remotely accessible, but access can be controlled. This will include data that is potentially proprietary, classified, or export controlled.

3. Updates to the database are permitted by approved users.

4. The data will be searchable and interactive.

5. The database will facilitate high performance computing within multi-physics modeling frameworks.

The basic functional requirements include the following:

1. Provide thermophysical data for salt mixtures using measured data and provide interpolation and extrapolation schemes between state properties (temperature) and compositions.

2. Salt compositions to be included are those with MSR design applicability: both fuel and coolant salts.

3. Thermophysical data includes, at a minimum, density, viscosity, heat capacity, and thermal conductivity in a range of MSR-relevant temperatures. Other variables can be added dependent on their significance for the evaluation.

4. Thermophysical data are included for relevant temperatures: at normal operation, which includes AOOs, ranging from the melting point, typically around $500^{\circ} \mathrm{C}$, to $1000^{\circ} \mathrm{C}$ (an approximate peak temperature expected for some anticipated operational occurrences but dependent on reactor design).

The database should be both interactive and exploratory and it should be developed for efficient computation. For the database to be used for interactive, exploratory use (personal computer or web page) a script language, such as Python, would be a suitable language to use as an interface. For the database to be used in high performance computing codes, these high-level scripting languages are too slow. The database will be designed for HPC with either (1) an interface can be created for interactive usage, or (2) 
a preprocessing code that generates an HPC database from a more interactive database. If the memory requirements or performance of this library are unsuitable for HPC applications, a replacement model (perhaps a database of interpolated values) will be implemented; a combination of models will be considered and studied.

\subsection{EXISTING STANDARDS AND DATABASES}

There is precedent for standard thermophysical databases that can be leveraged for the development a property database for molten salts. The following are a few examples of extensive databases that have wide acceptance in academia and industry. Many more examples of property databases exist. For example, the chemical and process engineers depend on extensive, proprietary databases for chemical separations, reactions, and transport such as Aspen Properties (AspenTech, 2001) and the DIPPR 801 database from the Design Institute for Physical Properties (DIPPR)(Wilding et al., 1998).

\subsubsection{REFPROP}

The REFPROP database is considered the gold standard in thermophysical databases and is maintained by the National Institute of Standards and Technology (NIST) (Lemmon, 1989). The NIST REFPROP database provides thermophysical property models for a variety of industrially important fluids and fluid mixtures, including accepted standards. The distribution of this database is through NIST (https://www.nist.gov/srd/refprop) and includes the FORTRAN source code so that it can be compiled and distributed, per license requirements, to customer specific applications. For example, there exists wrappers that permits querying the database from tools such as Python, MATLAB, Excel, Labview, and $\mathrm{C} / \mathrm{C}++$ (https://github.com/usnistgov/REFPROP-wrappers).

\subsubsection{COOLPROP}

CoolProp (http://www.coolprop.org/) is an opensource thermophysical property database with state-ofthe-art formulations for the thermophysical properties of fluids (Bell et al., 2014). It has many similarities to REFPROP though does not support quite as many fluids. One of the major limitations of CoolProp is that its support of fluid mixtures is still being developed and improved however it has integrated an interface to NIST REFPROP provided the user has a REFPROP license. Given the code is open-source $\mathrm{C}++$ library (https://github.com/CoolProp/CoolProp), can be wrapped for a given application (e.g., Python, MATLAB, and Modelica), and that CoolProp is widely used, it provides an excellent example of how, in many aspects, a modern, accessible molten salt database would be developed, maintained, and function.

\subsection{VALIDATION AND CONSISTENCY}

It is often the case that measured quantities taken from different sources may not be combined in a way that is consistent with either the original data or the intended use of combined measurements. Hence, a strong effort will be made to validate each entry under a variety of usage scenarios. This effort will also include comparisons of replicate (or similar) measurements and recognition of ranges of applicability. In addition, the original source documents will be referenced, and for unpublished or obscure sources, the entire document should be included.

\subsection{FUNCTIONAL NEEDS OF A THERMOPHYSICAL MOLTEN SALT DATABASE}

Simulation tools, from basic programming languages to system modeling tools to computational fluid dynamic codes, largely rely on the ability to query basic property data of the simulation fluid to perform the varying degrees of research, engineering, and design analysis. Specific codes and methods will at 
some point diverge in the information required just as varying levels of fluid data is available to describe the fluid behavior. Given the wide variety of fueled and non-fueled salts likely to be of interest to research, development, and design of molten salt reactors, the databases for thermophysical property interrogation must be robust in its ability to handle all potential fluids and thorough in providing available properties to users. The following is an initial list of high-level requirements of a thermophysical database. It is expected that additional work on database creation would extend this list to improve the databases usefulness and general applicability.

For the thermophysical data, a software library that provides an API to access a property database is required. In a full-core mutliphysics simulation, it is anticipated that $25-30 \%$ computational effort will be related to chemistry thermophysical property coupling. However, it is possible to have fast and slow options by making some approximations depending on the frequency of computation. For some computations, the species concentration is needed only at the hot and cold temperatures to bound the operation, but in others, information will be required on a node-by-node basis. Thus, thermophysical properties may need to be looked up very frequently (several times for each node at each time step). Thermochimica is set up as an API for code coupling. It reads Fortran formatted text files as part of the initial loading process and then uses it multiple times as needed. The format for the thermochemical data is already defined.

Mapping generally occurs from enthalpy to temperature, then from temperature to all other properties. The pressure differences expected within a reactor generally have limited impact on property values. The computation will specify the materials at the beginning of a time step and then evaluate that set of materials at multiple temperatures spanning the range of interest. Standard polynomials and table interpolation (or combinations of the two) are the likely forms of thermophysical data. The database will include provenance metadata on the physical properties, and it should provide experimental uncertainties when available. A method to treat missing data and report it to the analyst is needed. The ability to perturb the values in this database for sensitivity studies may also be valuable.

To improve computational efficiency, and to open the possibility of multithreaded/GPU implementations, it would be prudent for the interface to provide compositions, temperatures, and pressures for a batch of cells at a time, rather than just a single cell. This, for example, would allow consecutive Thermochimica solves to use the previous (and presumably similar in composition) cell as an initial solution for calculating chemical equilibria.

Representation inconsistencies between codes (i.e., ORIGEN, VERA-MSR, and Thermochimica, and TRANSFORM) must be handled. The use of nuclide information is the baseline representation as opposed to elemental inputs. Radionuclides are used directly in ORIGEN and VERA-MSR to get source spectra and intensity in each computational element of the reactor. Thermochimica accepts element input and a routine to collapse nuclides into elements is required.

The isotopic concentration of reactor salts is calculated using a mature depletion methodology (ORIGEN). The data and models used for this analysis are well-established for solid-fueled systems and are extendable to liquid-fueled systems with a few approximations to account for chemical processing. Depletion calculations account for fission, transmutations, nuclear decay, and isotopic removal and production.

The thermophysical database:

- Should enable varying levels of implementation for fluids of interest, from loose characterizations to fully validated and verified data (i.e., FLiBe and Fueled-FLiBe) 
- Should be extendable to fluids of differing complexities (i.e., single-phase and two-phase)

- Should be able to handle fluids of varying compositional mixtures (i.e., number of species between fluids and varying mole or mass fraction for the same fluid). The determination of species to be included in the composition for thermophysical property determination will be limited to the species that significantly impact the properties. This prioritization or filtering criteria must be established. Other species of interest to the user's applications that do not impact thermophysical properties will be handled external to the database at the user's discretion.

- Should enable additional fluids to be added without code restructure

- Should have queryable properties as a function of the proper state variables. The variety of properties available will depend on the base class for fluids of different complexities. For example, incompressible fluid mixtures will require functions to be a function of temperature and composition and will not be required to contain any information of phase transition properties. Two-phase fluids and compressible fluid mixtures on the other hand will, at a minimum, require two state variables (e.g., pressure and temperature) and a composition variable and will require information for phase transition information.

- Should not be required to couple the database with more complex codes for every required evaluation. For example, online simulation of the thermophysical database with Thermochemica. Necessary equations of state, table data, etc. for the thermophysical database would be prepopulated using more complex, computationally expensive codes offline.

- Should conform to established conventions/standards (RSICC/NIST/ASME).

- Should be operating system/platform agnostic. This requires the ability, in some form, to compile and distribute the database to a variety of end-uses such as Linux and Windows machines, desktop and web applications, and a variety of compilers (e.g., Intel, GCC, and Visual Studio).

- Should be efficiently implemented to limit computation cost of querying the database for properties.

\subsection{THERMOPHYSICAL PROPERTIES IN THE DATABASE}

There is a minimum set of information common to all fluids necessary for certain applications, e.g., thermal-hydraulic/transport calculations. An initial set of properties required of all fluids entered into the database include:

- Density and specific volume as a function of temperature and composition

- Specific heat capacity as a function of temperature and composition

- Specific enthalpy as a function of temperature and composition

- Thermal conductivity as a function of temperature and composition

- Dynamic viscosity as a function of temperature and composition

- Melting and boiling point as a function of composition

- Partial derivatives of these variables each with respect to the appropriate state variables

\subsection{DATABASE IMPLEMENTATION}

A preliminary Molten Salt Chemistry Database has been implemented on a secured data server at ORNL. The database repository is named "SaltChemProp." The database will house thermophysical property data for specific salt compositions, in addition to thermochemistry input files for specific salt families. Within the database, files can be produced and saved for specific concepts operated under any number of proposed operational histories. 
The initial storage location is an internal server hosted through ORNL using GitLab. This allows access to be controlled and proprietary and export-controlled information to be stored. This arrangement allows for online storage of large data files in a central storage location and also allows for a streamlined process to develop the repository and interfaces.

An open-source CoolProp repository is stored inside SaltChemProp. The CoolProp data files are stored in JSON format. JSON is not an efficient file storage format for accessing data on HPC systems. Based upon previous experience with neutronics data used in transport simulations on laptops, small clusters, and HPC systems, the ultimate format for the database files will be HDF5. HDF5 is a hierarchical data format that is portable and extensible. It supports an unlimited variety of datatypes and is designed for flexible and efficient input and output for high volume and complex data. The variables and format of the JSON files will be used for preliminary testing, but data will be ported to the more efficient and comprehensive HDF5 data files.

Within SaltChemProp folders store the source, documentation, and data. The data folder houses any open salt properties but also provide a place to insert extra repositories of proprietary data. The infrastructure will look for these proprietary repositories and give approved users access to the proprietary data through its interfaces. If proprietary data is not present, the infrastructure will give the user access to only the open salt properties. This structure allows a company or user to provide new salt data but retain control over who can access that data in a way that is seamless to the physics codes interfacing with the database.

The requirements for the structure and data included in the SaltChemProp database are as follows:

1. Needs to allow for efficient access on laptops, small clusters, and HPC machines.

2. Should be operating system/platform agnostic. This requires the ability, in some form, to compile and distribute the database to a variety of end-uses such as Linux and Windows machines, desktop and web applications, and a variety of compilers (e.g., Intel, GCC, and Visual Studio).

3. Should be efficiently implemented to limit computation cost of querying the database for properties and allow for extensibility.

4. Must interact efficiently with VERA-MSR and TF.

5. Should consist of wrappers for interfaces and underlying $\mathrm{C}++$ code for extensibility including Fortran, Python, and C.

6. Should include the raw data used for Thermochimica, the data produced from Thermochimica, raw thermal properties, and the processed thermophysical data.

\section{FUTURE NEEDS}

\subsection{DEVELOPMENT NEEDS}

\subsubsection{Transport Models}

Thermo-physical property measurements and salt isotopics directly influence reactor behavior. Salt thermodynamic properties describe the behavior of complexes within the salt. The missing elements of reactor evaluation are the transport models that describe how salt components move within the system. The forms of interest from salt operation within the reactor include gaseous products, solids, noble metals, and reactive elements as salts in solution. Other elements of interest include potential corrosion products 
from the structural materials (likely chromium, iron, and nickel) or elements that enter the salt through on-line or side stream processes. These elements may have limited impact on reactor behavior, but even trace amounts could have large impacts on associated processing systems or eventual waste forms.

Noble metals are expected to plate-out into the system and thus represent a static, growing, and distributed source term. Where they plate-out and if and when they leave a surface is important to understand. The five noble metals associated with solid fuels are $\mathrm{Mo}, \mathrm{Rh}, \mathrm{Ru}, \mathrm{Pd}$, and $\mathrm{Tc}$. For reactor concepts that utilize lithium or beryllium within the primary system salt, a significant quantity of tritium can be produced. Three elements considered primary drivers of off-site dose consequence are iodine, strontium, and cesium. Thus, the behavior of these specific elements must be understood. Therefore, for molten salt reactors, the following transport models require development:

1. Solid formation of salt constituents

2. Nucleation, growth, and transport of solids

3. Transport of non-reactive particles within the salt

4. The predominate noble metals ( $\mathrm{Mo}, \mathrm{Rh}, \mathrm{Ru}, \mathrm{Pd}$, and $\mathrm{Tc}$ )

5. Tritium

6. Iodine

7. Cesium

8. Strontium

\subsubsection{Severe Accidents Analysis}

Because of a lack of design detail, it is not yet possible to accurately predict severe accident progression within an MSR. However, it is possible to consider the end state of a severe accident progression: the primary system salt is significantly drained out of the primary system. In this scenario for a liquid-fueled MSR, the radioactive products are already distributed throughout the plant (effluent systems), the salt, or on primary system structures.

It is likely the salt constituents that normally leave or would be removed from the salt under normal operating conditions are mostly removed at the time of any operational incident and additional models are not required. Additional issues relate to what occurs when a salt goes to significantly higher or lower temperatures, reduced flow rates, or potentially drains out of the primary system. If the temperature increases, otherwise soluble constituents could begin to evolve out of the system. Systems to manage effluents, such as the off-gas systems, must be designed to handle the total anticipated loading and the maximum loading rate with sufficient margin.

\subsubsection{Expansion to Chemistry Separation Processes}

Liquid fueled molten salt reactors operate by consuming fission products directly within the salts. This process adds fission products, along with activation and corrosion products, to the salt over time. As fuel is consumed and as neutron absorption increases, additional fissile materials must be added. Liquid fueled MSRs will have salt constituents that leave the salt at a liquid-gas interface and this loss of material must be accurately modeled. Other constituents may move to the wall where they can adhere, or, as in the case of tritium, may diffuse out. The balance of constituents will remain in the salt.

Constituents within the salt can be allowed to remain or they can be actively separated. The separation process can be from active systems, such as the use of inert gas sparging to increase migration of gas species out of the salt, or from additional ancillary chemical processing; the chemistry used to facilitate removals and the systems that implement that system must also be modeled. At issue are the separation efficiencies, the cross-contamination, and the energy balances associated with the processes. 


\subsubsection{Thermochemical Measurements}

In FY18, the preliminary modeling framework was established. Fiscal year 19 activities will focus on establishing experimental capabilities and generating prioritized data summarized in Table 14.

Table 14 Relevant MSR thermochemical data and appropriate measurement technique.

\begin{tabular}{|l|l|}
\hline \multicolumn{1}{|c|}{ Data } & \multicolumn{1}{c|}{ Technique } \\
\hline Vapor pressures & Knudsen effusion, transpiration \\
\hline Phase equilibria & $\begin{array}{l}\text { Differential Scanning Calorimetry (DSC), } \\
\text { Differential Thermal Analysis (DTA) } \\
\text { Mass Spectrometry (MS) }\end{array}$ \\
\hline Heat capacities & DSC \\
\hline Heats of fusion & DSC, other calorimetry \\
\hline Corrosion behavior & Redox potentials, exposure testing, etc. \\
\hline
\end{tabular}

\subsubsection{Chemistry Model Development}

Currently, CALPHAD modeling efforts have focused on pseudobinaries such as the $\mathrm{KCl}-\mathrm{MgCl}_{2}$. However, an assessment of the integral and fundamental ternaries like $\mathrm{K}-\mathrm{Mg}-\mathrm{Cl}$ are needed to build functional multicomponent system models. Key work is needed to extend the MQM to include excess metal species, for example $\mathrm{K}$ and $\mathrm{Mg}$ in the $\mathrm{K}-\mathrm{Mg}-\mathrm{Cl}$ ternary. In FY18, the framework was developed to do just that; results are shown in Figure 20. 


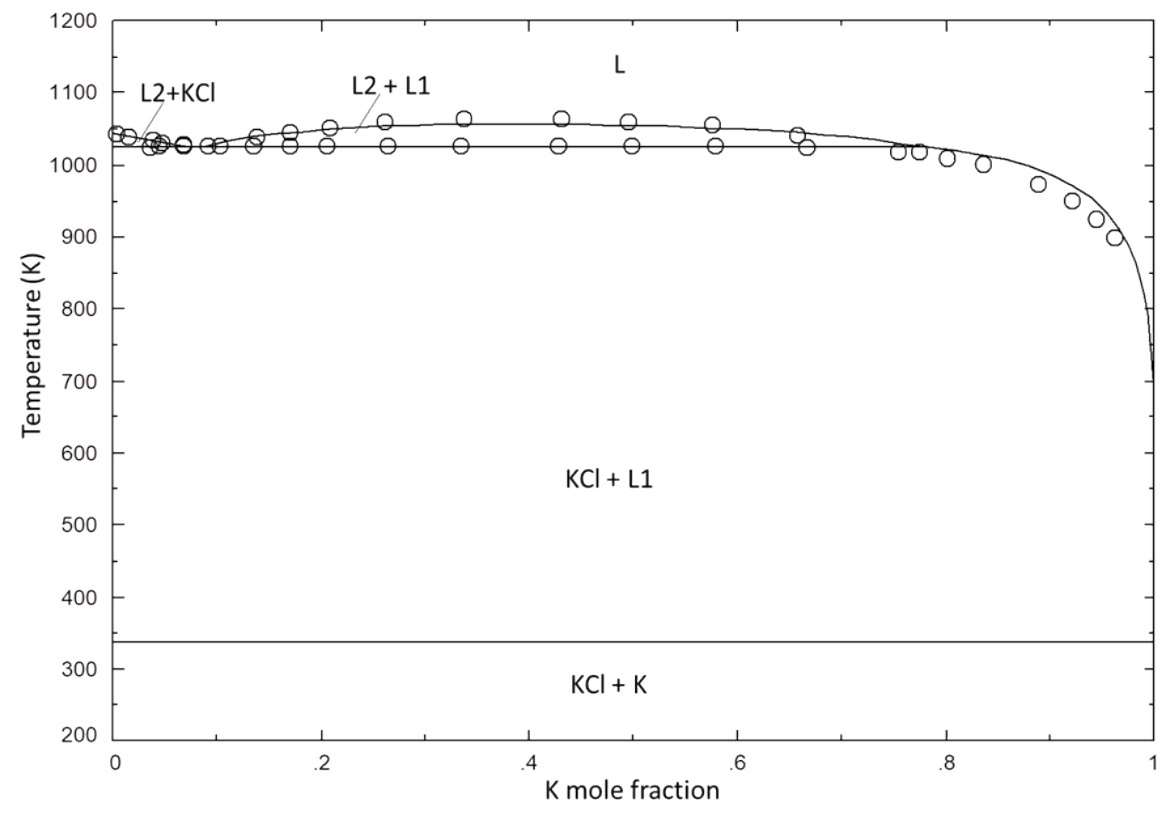

Figure 20 Computed K-KCl phase diagram using the solution model for the melt developed in this work with the thermodynamic values from the FactSage 7.1 database [77] for pure components and stoichiometric compounds. Symbols are experimental points from [101].

The same approach should be applied to other metal chloride and fluoride systems to facilitate high fidelity multicomponent CALPHAD representations of molten salts and more reliable reactor performance simulations.

\subsubsection{Thermochimica Development}

Exploration of interfacing ORIGEN and Thermochimica with COBRA-TF is underway. Simulations for any particular grid element and time will again start with ORIGEN, to provide the isotopic physics calculations for the fuel. Individual isotopes are incremented or decremented by decay, depletion and generation. As output, ORIGEN will provide the specific power due to neutron capture and fission as well as the mass of all isotopes under consideration. The results for isotopic masses are distilled and Thermochimica is called. Specific power data will be sent to COBRA-TF to provide source term information for heat transfer calculations.

Thermochimica provides the molar quantities of phases stable at thermodynamic equilibrium, as well as the chemical potentials of the system components, and the molar heat capacity, entropy, enthalpy and Gibbs energy of the system. Heat capacity, enthalpy and phase distribution \& speciation will be sent to COBRA-TF. As input, Thermochimica requires hydrostatic pressure, temperature, the mass of each element (from ORIGEN) and a thermodynamics database, for example [7]. Temperature and pressure will be received from COBRA-TF. The thermodynamics databases available for molten salt reactors will require an upgrade to Thermochimica's capabilities. The individual phases will be sorted into gas, liquid and solid precipitates, to be submitted to COBRA-TF. Heat capacity and enthalpy of the reactions will be used by COBRA-TF to calculate the temperature, which will be needed by Thermochimica to calculate the next iteration. After the completion of the time step, the calculation will repeat, starting with ORIGEN. See Figure 1 for the flows of information between physics codes. 
Additional applications are being considered. While material properties such as viscosity, enthalpy and heat capacity could be approximated with some simplified models, a multi-physics simulation with Thermochimica is required to calculate these properties for phase fractions for multi-phase flow. In addition to the preceding, Thermochimica has the potential to provide boundary conditions and material properties for structural mechanics and heat transfer calculations [1]. Thermochimica's unique capabilities make it especially suitable for multi-physics simulations such as irradiated fuel in coolant.

Special attention will be paid to boundary conditions, where certain metals will plate-out (surface corrosion). This is an area of interest to the authors, as the decay heat from plated noble metals may cause thermal failure of the pipe in event of a loss of cooling. Another particular area of interest is the use of $\mathrm{He}$ gas to bubble up poisons such as Xe or radiotoxins such as I.

Potential future work with Thermochimica may include the following activities:

- Code maintenance.

- Supporting integration into the multi-physics framework (e.g., provide appropriate quantities for input to COBRA).

- Interfacing with surface corrosion models.

- Development of numerical methods to accelerate convergence to avoid bottlenecking.

\subsubsection{First Principles and Machine Learning Approach to Thermodynamic Values}

The CALPHAD approach relies on a suite of data (either experimentally measured or theoretical values from simulation) to evaluate adjustable model parameters. While some types of data are available and easily measured, others are difficult or impossible to determine experimentally.

As it has been demonstrated over the last couple decades, first-principles density functional theory (DFT) calculations can successfully predicted thermochemical values of condensed phases. A similar benefit is anticipated in predicting thermochemistry of chlorine and fluorine phases to facilitate CALPHAD assessments of molten salt systems.

Data analytics approaches for predicting thermochemical values deserves attention as well. Figure 21 shows predicted formation enthalpies and entropies at $25^{\circ} \mathrm{C}$ of complex oxides using a machine learning approach. Linear regression and Bayesian Ridge regression models have been trained with about 100 experimentally measured thermochemical data using a key descriptor, polyhedra, as illustrated in Figure 22 . The accuracy of trained model for formation entropy is slightly lower $(96.7 \%)$ than that of enthalpy $(99.7 \%)$, however, these are remarkably accurate surrogate models. It should be noted here that a number of oxides in the training dataset are transition and rare-earth metal oxides, which continue to be problematic when computing thermochemical values with DFT. Well-trained machine learning models make it now possible to accurately predict thermochemistry of phases with correlated electronic structures. 


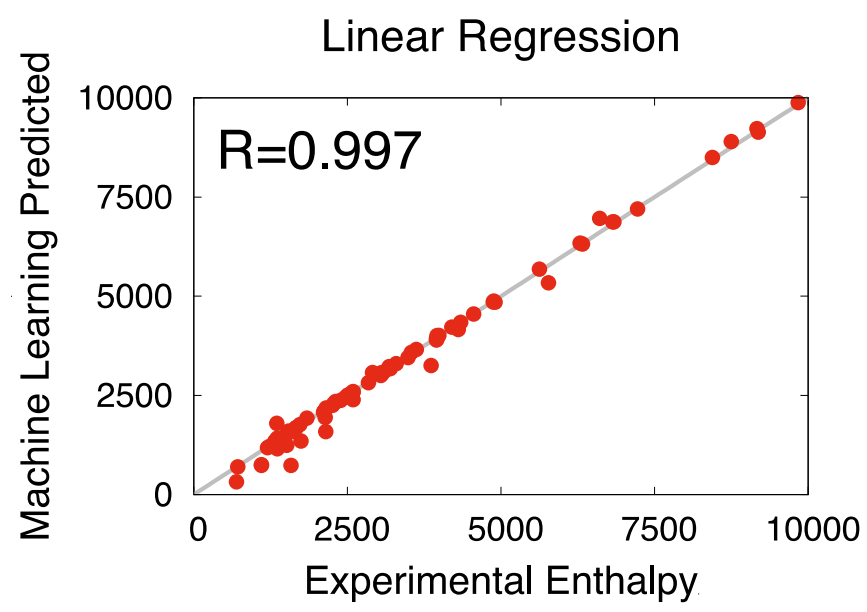

Bayseian Ridge Regression

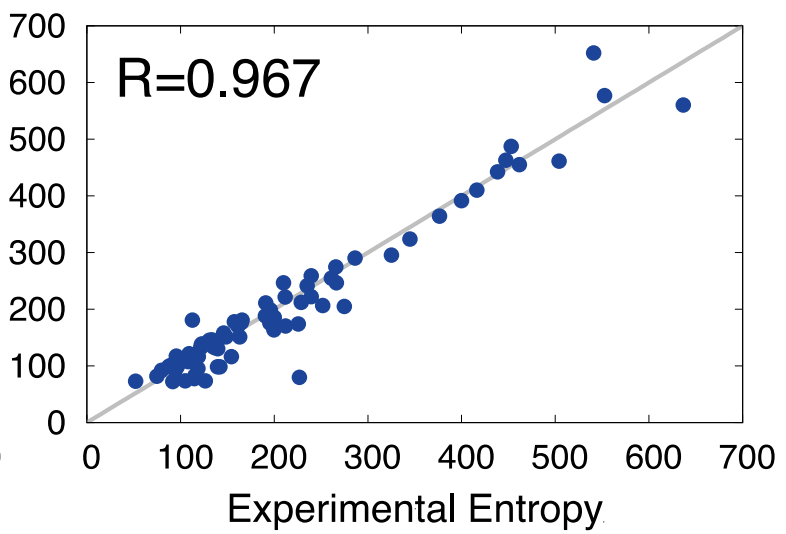

Figure 21 Predicted enthalpies (left) and entropies (right) of complex oxides via machine learning approach. About 100 oxides thermochemistry data obtained from experiments have been used to train linear regression model to predict formation enthalpies and Bayesian Ridge regression model to predict formation entropies at $25^{\circ} \mathrm{C}$, respectively.

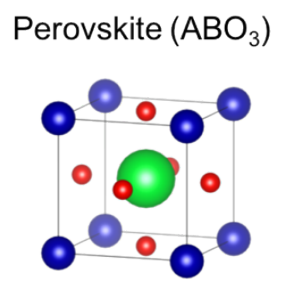

Brownmillerite $\left(\mathrm{ABO}_{2.5}\right)$

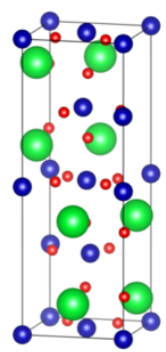

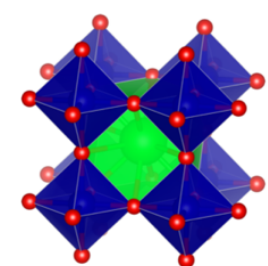

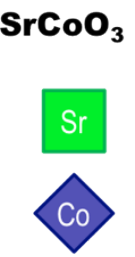

$\Delta \mathrm{H}_{\mathrm{f}}=-712 \mathrm{~kJ} / \mathrm{mol}$
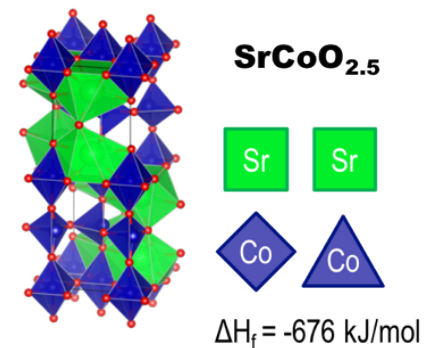
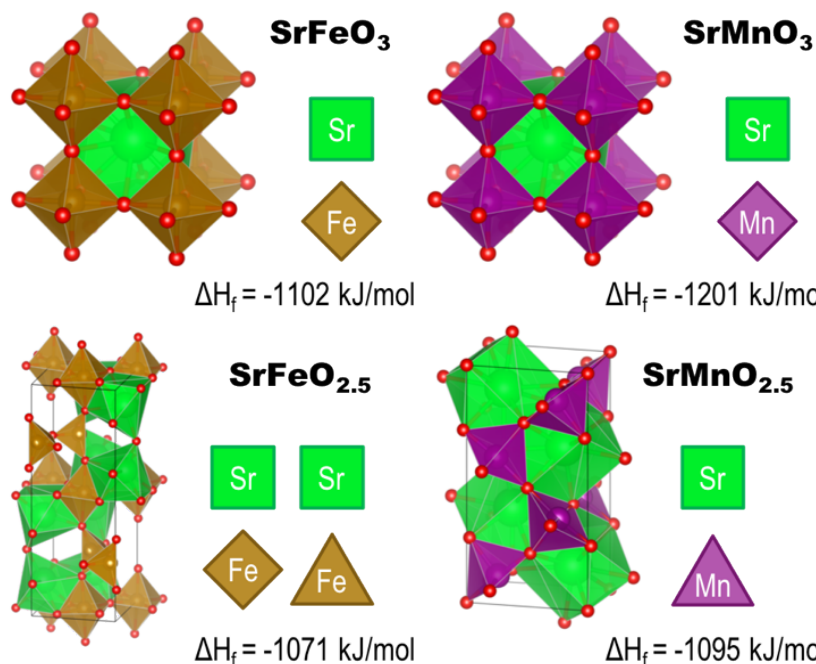

$\Delta \mathrm{H}_{\mathrm{f}}=-1102 \mathrm{~kJ} / \mathrm{mol}$

$\Delta \mathrm{H}_{\mathrm{f}}=-1201 \mathrm{~kJ} / \mathrm{mol}$

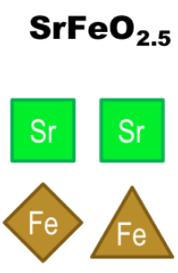

$\Delta H_{\mathrm{f}}=-1071 \mathrm{~kJ} / \mathrm{mol}$

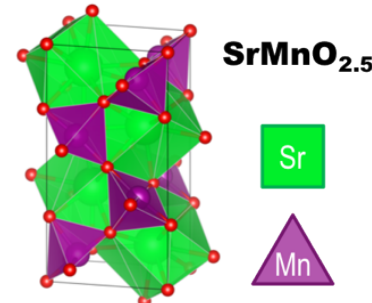

$\Delta \mathrm{H}_{\mathrm{f}}=-1095 \mathrm{~kJ} / \mathrm{mol}$

Figure 22 Polyhedra-based features used to train machine learning models to predict thermochemistry of complex oxides. Examples shown here are perovskite and brownmillerite phases which have similarities in both stoichiometry and crystal structures. Polyhedra can simultaneously capture composition (with oxidation states of metal ions) and crystal structural information.

It is anticipated that a machine learning technique using descriptors like bond types, bond lengths, and bond angles for example, will be able to accurately predict thermochemistry of vapor species, which is largely missing in molten salt systems. 


\subsubsection{Thermophysical Property Models and Measurements}

The ultimate goal of the thermophysical properties database development activity is to implement a stateof-the-art functional information tool (database/module) that provides the fuel/coolant salt properties needed to model MSR performance as a function of temperature, pressure and salt composition. The database will be integrated into the system-level modeling and simulation tools being developed as part of the MSR campaign to assess and optimize the performance of particular MSR concepts. The specific content of the database will include experimentally measured thermophysical properties (e.g., density, viscosity, thermal conductivity) as well as predictive functions for the interpolation and extrapolation of those properties. The envisioned relationship between the thermophysical database/module and other components of the MSR modeling and simulation framework is shown Figure 1.

Relevant literature was reviewed and summarized as an initial step in developing the thermophysical database/module. The results of this initial literature summary identify specific data gaps and provide objectives for future R\&D activities in this area. For example, the technical context for A set of R\&D activities addressing those gaps is presented schematically in Figure 23 and relevant experimental data sets and comparisons of thermophysical property models to be enhanced by those activities are provided in Section 2.2.

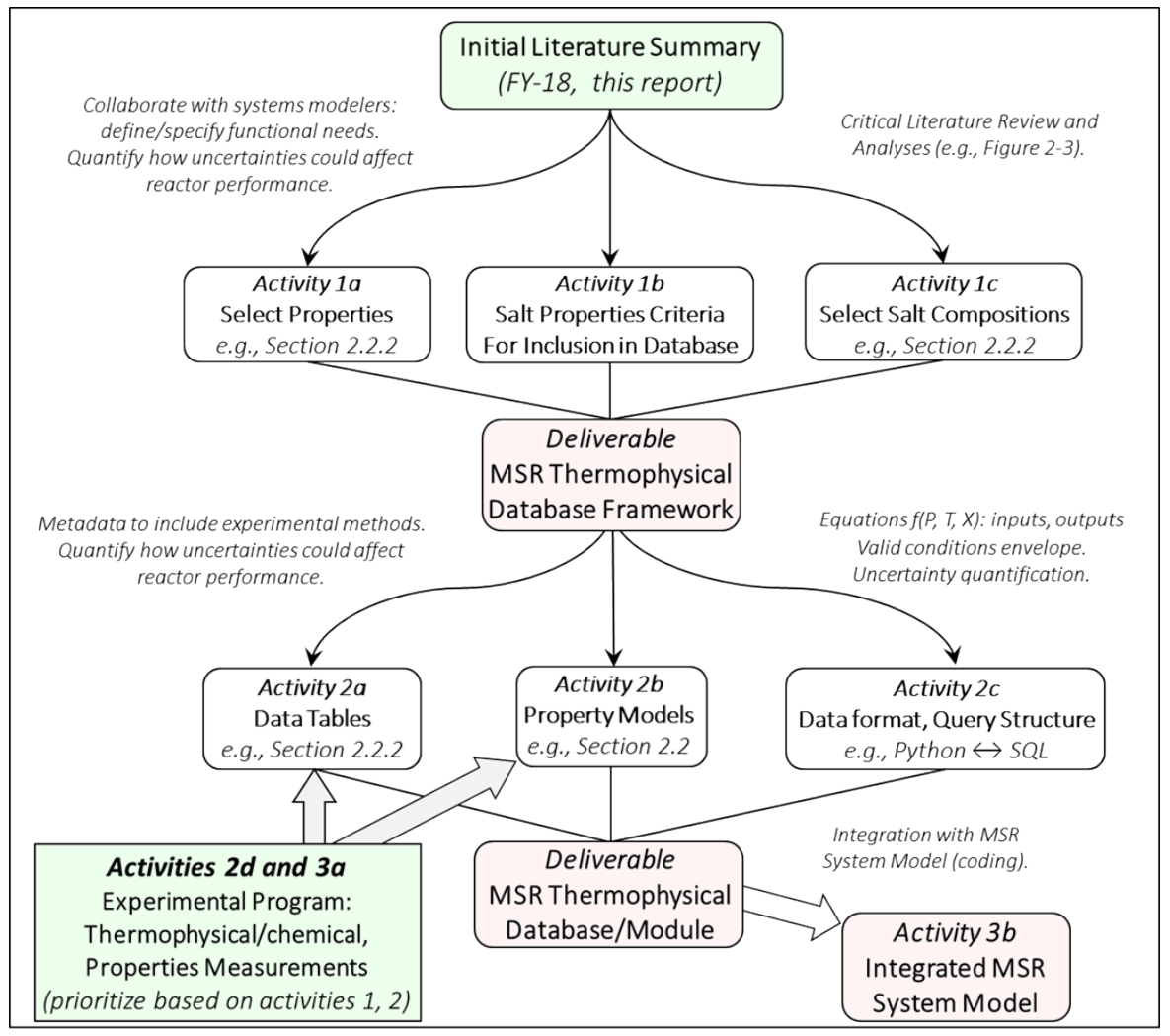

Figure 23 Diagram showing gaps addressed by $R \& D$ activities to develop a state of the art thermophysical database/module for design and assessments of MSRs.

Database development will require close coordination and abundant discussion between molten salt reactor developers, systems engineers, modeling and simulation experts, chemists and chemical engineers. A general flow diagram depicting the activities for database development identified in Figure 23 are summarized as follows: 
- Activity 1a: Select properties to be included in thermophysical database,

$\circ$ Define and specify functional data needs for design and assessments of MSR systems

- Identify data quality required for MSR modeling and simulation

- Identify and assess salt property model needs to enable salt property extrapolation and interpolation

- Identify and evaluate data sensitivity to quantify how uncertainty ranges for selected thermophysical properties affect modeled reactor performance

- Activity $1 b$ : Determine the criteria used to rank, select and prioritize use of fuel/coolant and coolant salt systems

- Identify and review availability and quality of salt property values for MSR systems currently under development or proposed for development

○ Identify alternative salt systems that may facilitate MSR system development

- Activity 1c: Select salt compositions to be included in thermophysical database.

- Identification and assessment of available literature data

- Identify additional data needs for database development

- Preliminary review of available literature for salt systems identified

- Deliverable: Document defining the framework for the molten salt thermophysical properties database using existing example data or place-holder values and models.

- Activity 2a: Populate database with critically reviewed data

- Identify and document methods for critical data review

- Critically review salt property data for base salt systems identified in Activity 1c

- Compile Metadata for database entries including experimental methods, quantified uncertainties if available and an uncertainty assessment

- Activity 2b: Input established property models that can be used for interpolation and extrapolations of property values outside of the experimental conditions

- Define inputs and outputs of property models

- Integrate the thermophysical properties database with the thermochemical solver and the MSR model needs

- Activity $2 c$ Determine most efficient data format, query structure

○ Database will likely be implemented in Python to facilitate calculations with an SQL tiein for the databasing aspects.

- Deliverable: Fully functional MSR thermophysical module/database

- Activity $2 d$ : Plan experimental program to fill key data gaps identified in thermophysical database

- Develop test plans based on Activities $2 \mathrm{a}$ and $2 \mathrm{~b}$ and implementation of inter-laboratory QA guidelines, salt purity criteria, standard methods, best practices, etc.

- Activity 3a: Perform experimental work to fill data gaps in database

$\circ$ Conduct experiments within programmatic QA guidelines, sharing samples, reference materials and best practices between laboratories

- Conduct critical review of experimental data and upload to database with quality figure of merit

- Activity 3b: Integrate MSR thermophysical databased with multi-physics MSR optimization/performance codes.

- Collaborate with modelers to efficiently integrate the thermophysical database/module into system codes that can be used to support MSR design, optimization, and licensing

- Campaign deliverable: Integrated MSR system model that includes thermophysical database/module

The preliminary literature review summarized in Section 2 included critical reviews of existing salt properties databases. For example, [19] provides an assessment of a thermophysical properties for 
candidate coolant salts for the Advanced High-Temperature Reactor concept. This review serves as a good starting point and guide for the early stages of thermophysical properties database development activities for the MSR Campaign. The review results of [19] are summarized in Figure 24.

\begin{tabular}{|c|l|l|l|}
\hline $\begin{array}{c}\text { Predictive Model } \\
\text { Existing Database }\end{array}$ & Accurate & Approximate & Information Gap \\
\hline Accurate & Density & $\begin{array}{c}\text { Melting Point } \\
\text { Vapor Pressure } \\
\text { Viscosity }\end{array}$ \\
\hline $\begin{array}{c}\text { Data Gap: } \\
\text { Dard Measurement } \\
\text { Method Exist }\end{array}$ & Heat Capacity & \\
\hline $\begin{array}{c}\text { Data Gap: } \\
\text { Difficult to Measure }\end{array}$ & & $\begin{array}{c}\text { Thermal } \\
\text { Conductivity }\end{array}$ \\
\hline
\end{tabular}

Figure 24 Qualitative assessment of the thermophysical properties database and predictive modeling methods for high temperature coolant salts by [19] (adapted).

The key finding of the initial literature assessment is that there is a strong need for an experimental program focused on measuring the thermophysical properties for key binary and ternary salt systems. The experimental program would include activities to fill gaps in the existing salt properties database (e.g., in Table 1, Table 2, Table 3, and Table 4) and would validation of the analytical expressions (e.g., Table 5) used for interpolation and extrapolation of property values for compositionally complex salts (e.g., fission product- and actinide-rich salts). The following list identifies the main properties that need to be quantified to support modeling MSR systems and represent the core salt properties addressed in a functional database. Also listed are the preferred measurement methods that have been recommended in recent state-of-the-art literature reviews (Serrano [23], Capelli [41] and Fortner et al. [66]

- Salt composition, phase equilibria

○ Differential Scanning Calorimetry (DSC),

- Differential Thermal Analysis (DTA)

- Mass Spectrometry (MS)

- Heats of fusion

- DSC, other calorimetric methods

- Heat capacities

- DSC, other calorimetric methods

- Thermal conductivity

- Laser flash method (thermal diffusivity)

- Density

- Archimedes method

- Vapor pressure

- Knudsen effusion

- Transpiration

- Volume Expansion

○ Archimedes method

- Viscosity

○ Rotating spindle viscometer, 
- Oscillating cup viscometer

The target uncertainties levels (e.g., accuracy and precision requirements) for each property measurement will be determined by iterative sensitivity analyses using preliminary MSR performance codes being developed as part of this campaign e.g., references $[6,7]$. In these sensitivity analyses, reactor performance will be calculated using different values of properties such as density, viscosity, and thermal conductivity within some reasonable ranges and compared to identify the relative sensitivity to each parameter. In addition to providing target uncertainty levels for property measurements, the results will establish priorities for properties evaluated in the experimental program.

\section{ACKNOWLEDGEMENTS}

This research was funded by the US Department of Energy Office of Nuclear Energy Advanced Reactor Technology program. The contribution from Dr. M.H.A Piro and B. Fitzpatrick at the University of Ontario Institute of Technology was funded, in part, by the Canada Research Chairs program (950231328) of the Natural Sciences and Engineering Research Council of Canada.

\section{REFERENCES}

[1] B. R. Betzler, N. R. Brown, B. Feng, F. Heidet, C. Rabiti, T. Sofu, A. J. Wysocki, Molten Salt Reactor Modeling and Simulation Functional Needs, Oak Ridge National Laboratory, NTRD-MSR-2018- 000450 (2018)

[2] Z. Taylor, R. Salko, B. Collins, G. Maldonado, Implementation of General Species Transport

Capability into VERA-CS for Molten Salt Reactor Analysis, Driving the Future of Nuclear Technology, American Nuclear Society Annual Meeting, (2018)

[3] S. M. Bowman, Nucl. Technol., 174 (2) (2011) 126-48.

[4] I. C. Gauld, G. Radulescu, G. Ilas, B. D. Murphy, M. L. Williams, D. Wiarda, Nucl. Technol., 174 (2) (2011) 169-95.

[5] M. D. DeHart, S. M. Bowman, Nucl. Technol., 174 (2) (2011) 196-213.

[6] B. R. Betzler, J. J. Powers, A. Worrall, Annals of Nuclear Energy, 101 (2017) 489-503.

[7] B. R. Betzler, J. J. Powers, N. R. Brown, B. J. Reardon, Molten Salt Reactor Neutronics Tools in SCALE, International Conference on Mathematics \& Computational Methods Applied to Nuclear Science and Engineering, Jeju, Korea; April 16-20, 2017, (2017)

[8] D. A. Petti, R. Hill, J. Gehin, e. al., Advanced Demonstration and Test Reactor Options Study, Idaho National Laboratory (INL), Idaho Falls, ID (United States), (2017)

[9] M. Drouin, B. J. Wagner, J. Lehner, V. Mubayi, Historical Review and Observations of Defense-indepth, US Nuclear Regulatory Commission, Office of Nuclear Regulatory Research, 2016.

[10] D. Samuel, IAEA Internship Report, INPRO COOL, (2009)

[11] C. W. Forsberg, P. F. Peterson, R. Kochendarfer, Design options for the advanced high-temperature reactor, 8,Proceedings of ICAPP, (2008) 8-12.

[12] T. Auger, G. Barreau, J.-P. Chevalier, X. Doligez, S. Delpech, H. Flocard, B. Haas, D. Heuer, E. Merle-Lucotte, PACEN CNRS, Summary of Activities) June, (2008)

[13] K. Furukawa, Y. Kato, T. Kamei, International work shop on Thorium utilization for sustainable development of nuclear energy, Beijing, China, (2007) 8.

[14] W. Grimes, Reactor Chemistry Division Annual Progress Report for Period Ending December 31, 1965, Oak Ridge National Laboratory, (1966) 27.

[15] O. Beneš, R. Konings, J. Fluorine Chem., 130 (1) (2009) 22-9.

[16] V. Ignatiev, O. Feynberg, A. Myasnikov, R. Zakirov, Reactor physics \& fuel cycle analysis of a molten salt advanced reactor transmuter, 3,ICAPP, (2003) 4-7. 
[17] D. Ingersoll, Status of preconceptual design of the advanced high-temperature reactor (AHTR), ORNL, (2004)

[18] D. Williams, Assessment of candidate molten salt coolants for the NGNP/NHI heat-transfer loop, Oak Ridge National Laboratory (ORNL), Oak Ridge, TN (United States), (2006)

[19] D. Williams, Assessment of candidate molten salt coolants for the advanced high temperature reactor (AHTR), ORNL, (2006)

[20] C. W. Forsberg, P. F. Peterson, D. F. Williams, Practical aspects of liquid-salt-cooled fast-neutron reactors, Proceedings of the 2005 International Conference on Advances in Nuclear Power Plants, Seoul, Korea, May 15-19, (2005)

[21] C. Latge, Alternative coolants for intermediate loops of innovative SFR, ALISIA Info Meeting, Paris, France; (2018)

[22] O. Benes, R. J. M. Konings, J. Fluorine Chem., 130 (1) (2009) 22-9.

[23] R. Serrano-López, J. Fradera, S. Cuesta-López, Chemical Engineering and Processing: Process Intensification, 73 (2013) 87-102.

[24] M. S. Sohal, M. A. Ebner, P. Sabharwall, P. Sharpe, Engineering database of liquid salt thermophysical and thermochemical properties, Idaho National Laboratory (INL), (2010)

[25] J. C. Gehin, D. E. Holcomb, G. F. Flanagan, B. W. Patton, R. L. Howard, T. J. Harrison, Fast Spectrum Molten Salt Reactor Options, Oak Ridge National Laboratory (ORNL), (2011)

[26] A. Mourogov, P. Bokov, Energy conversion and management, 47 (17) (2006) 2761-71.

[27] C. Renault, M. Delpech, MOST Final Report, European Commission, (2005)

[28] V. Ignatiev, A. Merzlyakov, V. Afonichkin, V. Khokhlov, A. Salyulev, Transport properties of molten-salt reactor fuel mixtures: the case of $\mathrm{Na}, \mathrm{Li}, \mathrm{Be} / \mathrm{F}$ and $\mathrm{Li}, \mathrm{Be}, \mathrm{Th} / \mathrm{F}$ salts, Seventh Information Exchange Meeting on Actinide and Fission Product Partitioning and Transmutation, 14th-16th October, Jeju, Republic of Korea, (2002)

[29] V. Khokhlov, V. Ignatiev, V. Afonichkin, J. Fluorine Chem., 130 (1) (2009) 30-7.

[30] G. Janz, F. Dampier, G. Lakshminarayanan, P. Lorenz, R. Tomkins, MOLTEN SALTS: VOLUME I. ELECTRICAL CONDUCTANCE, DENSITY, AND VISCOSITY DATA, Rensselaer Polytechnic Inst., Troy, NY, (1968)

[31] G. Janz, U. Krebs, H. Siegenthaler, R. Tomkins, J. Phys. Chem. Ref. Data, 1 (3) (1972) 581-746.

[32] G. Janz, R. Tomkins, J. Phys. Chem. Ref. Data, 12 (3) (1983) 591-815.

[33] G. Janz, R. Tomkins, C. Allen, J. Downey Jr, S. Singer, J. Phys. Chem. Ref. Data, 6 (2) (1977) 409596.

[34] G. J. Janz, Physical Properties Data Compilations Relevant to Energy Storage: Molten salts: data on additional singe and multi-component salt systems, US Department of Commerce, National Bureau of Standards, 1981.

[35] G. J. Janz, Thermodynamic and transport properties for molten salts: correlation equations for critically evaluated density, surface tension, electrical conductance, and viscosity data, Amer Inst of Physics, 1988.

[36] G. J. Janz, C. B. Allen, J. R. Downey Jr, R. Tomkins, Physical properties data compilations relevant to energy storage. I. Molten salts: Eutectic Data, NATIONAL STANDARD REFERENCE DATA SYSTEM, (1978)

[37] G. J. Janz, C. Dijkhuis, G. Lakshminarayanan, R. Tomkins, J. Wong, MOLTEN SALTS. VOLUME 2. SECTION 1. ELECTROCHEMISTRY OF MOLTEN SALTS: GIBBS FREE ENERGIES AND EXCESS FREE ENERGIES FROM EQUILIBRIUM-TYPE CELLS. SECTION 2. SURFACE TENSION DATA, Rensselaer Polytechnic Inst., Troy, NY, (1969)

[38] G. J. Janz, G. Gardner, U. Krebs, R. Tomkins, J. Phys. Chem. Ref. Data, 3 (1) (1974) 1-115.

[39] G. J. Janz, R. Tomkins, C. Allen, J. Downey Jr, G. Garner, U. Krebs, S. K. Singer, J. Phys. Chem. Ref. Data, 4 (4) (1975) 871-1178.

[40] G. J. Janz, R. P. Tomkins, J. Phys. Chem. Ref. Data, 9 (4) (1980) 831-1022.

[41] E. Capelli, (2012) 
[42] C. W. Forsberg, Molten-salt-reactor technology gaps, Proc. 2006 International Congress on Advances in Nuclear Power Plants (ICAPP'06), (2006) 4-8.

[43] D. Olander, J. Nucl. Mater., 300 (2-3) (2002) 270-2.

[44] M. R. Zaghloul, D. K. Sze, A. R. Raffray, Fusion science and technology, 44 (2) (2003) 344-50.

[45] S. Cantor, PHYSICAL PROPERTIES OF MOLTEN-SALT REACTOR FUEL, COOLANT, AND

FLUSH SALTS, Oak Ridge National Lab., Tenn., (1968)

[46] R. Konings, L. Morss, J. Fuger, Springer, Dordrecht, 4 (2006) 2113-224.

[47] W. Grimes, CHEMICAL RESEARCH AND DEVELOPMENT FOR MOLTEN-SALT BREEDER REACTORS, Oak Ridge National Lab., Tenn., (1967)

[48] E. Veliyulin, A. Voronel, H. Oye, J. Phys.: Condens. Matter, 7 (25) (1995) 4821.

[49] S. Cohen, T. Jones, Viscosity measurements on molten fluoride mixtures, Oak Ridge National Lab., Tenn., (1957)

[50] J. Cooke, Experimental Determinations of the Thermal Conductivity of Molten Fluoride Mixtures, Oak Ridge National Laboratory, (1966)

[51] J. Cooke, Development of the variable-gap technique for measuring the thermal conductivity of fluoride salt mixtures, OAK RIDGE NATIONAL LAB., TENN., (1973)

[52] C. Gabbard, Reactor power measurement and heat transfer performance in the Molten Salt Reactor Experiment, Oak Ridge National Lab., (1970)

[53] X.-H. An, J.-H. Cheng, H.-Q. Yin, L.-D. Xie, P. Zhang, Int. J. Heat Mass Transfer, 90 (2015) 872-7.

[54] C. Nieto de Castro, TEMPMEKO 2004 - 9th Symposium on Temperature and Thermal

Measurements in Industry and Science, Cavtat-Dubrovnik,Croatia; (2004) 1-9.

[55] K. Cornwell, J. Phys. D: Appl. Phys., 4 (3) (1971) 441.

[56] V. Khokhlov, Light Metals, (1998)

[57] A. Bengtson, H. Nam, S. Saha, R. Sakidja, D. Morgan, Comput. Mater. Sci., 83 (2014) 362-70.

[58] H. Nam, A. Bengtson, K. Vörtler, S. Saha, R. Sakidja, D. Morgan, J. Nucl. Mater., 449 (1-3) (2014)

148-57.

[59] H. O. Nam, D. Morgan, J. Nucl. Mater., 465 (2015) 224-35.

[60] M. M. El-Wakil, American Nuclear Society, (1978)

[61] J. Ambrosek, M. Anderson, K. Sridharan, T. Allen, Nucl. Technol., 165 (2) (2009) 166-73.

[62] F. P. Incropera, D. P. DeWitt, Fundamentals of Heat and Mass Transfer, Australia, John Wiley \& Sons, 2002.

[63] C. F. Bonilla, Comparison of Coolants, In: H Etherington editor Nuclear Engineering Handbook; 1958, pp. 9-90.

[64] E. Compere, S. Kirslis, E. Bohlmann, F. Blankenship, W. Grimes, Fission Product Behavior in the Molten Salt Reactor Experiment, Oak Ridge National Lab., Tenn.(USA), (1975)

[65] A. Houtzeel, F. Dyer, STUDY OF FISSION PRODUCTS IN THE MOLTEN-SALT REACTOR

EXPERIMENT BY GAMMA SPECTROMETRY, Oak Ridge National Lab., Tenn., (1972)

[66] J. Fortner, M. Rose, M. Williamson, Standard Methods for Heat Capacity and Thermal Conductivity Measurements for Molten Salts, (2018)

[67] CALPHAD, http://www.thermocalc.com/academia/researchers/calphad/

[68] A. Pelton, S. Degterov, G. Eriksson, C. Robelin, Y. Dessureault, Metallurgical and Materials

Transactions B, 31 (4) (2000) 651-9.

[69] P. Chartrand, A. D. Pelton, Metallurgical and Materials Transactions A, 32 (6) (2001) 1417-30.

[70] P. Chartrand, A. D. Pelton, Metallurgical and Materials Transactions A, 32 (6) (2001) 1397-407.

[71] A. D. Pelton, P. Chartrand, Metallurgical and Materials Transactions A, 32 (6) (2001) 1355-60.

[72] A. D. Pelton, P. Chartrand, G. Eriksson, Metallurgical and Materials Transactions A, 32 (6) (2001)

1409-16.

[73] N. S. Patel, V. Pavlik, M. Boca, Critical Reviews in Solid State and Materials Sciences, 42 (1)

(2017) 83-97. 
[74] K. Fukumoto, R. Fujimura, M. Yamawaki, Y. Arita, Journal of Nuclear Science and Technology, 52 (10) (2015) 1323-7.

[75] J. R. Keiser, Status of Tellurium-Hastelloy N Studies in Molten Fluoride Salts, Oak Ridge National Laboratory, (1977)

[76] C. W. Bale, E. Belisle, P. Chartrand, S. A. Decterov, G. Eriksson, A. E. Gheribi, K. Hack, I. H. Jung, Y. B. Kang, J. Melancon, A. D. Pelton, S. Petersen, C. Robelin, J. Sangster, P. Spencer, M. A. Van Ende, CALPHAD, 55 (2016) 1-19.

[77] C. Bale, P. Chartrand, S. Degterov, G. Eriksson, K. Hack, R. B. Mahfoud, J. Melançon, A. Pelton, S. Petersen, CALPHAD, 26 (2) (2002) 189-228.

[78] M. H. A. Piro, S. Simunovic, T. M. Besmann, B. J. Lewis, W. T. Thompson, Computational Materials Science, 67 (2013) 266-72.

[79] R. Gauntt, MELCOR 1.8.5 Modeling Aspects of Fission Product Release, Transport and Deposition Sandia National Laboratories, SAND2010-1635 (2010)

[80] O. Benes, R. J. M. Konings, J. Alloys Compd., 452 (1) (2008) 110-5.

[81] O. Benes, R. J. M. Konings, J. Nucl. Mater., 435 (1-3) (2013) 164-71.

[82] J. P. M. van der Meer, R. J. M. Konings, K. Hack, H. A. J. Oonk, Chem. Mater., 18 (2) (2006) 510-7.

[83] A. Degtyarev, A. Myasnikov, L. Ponomarev, Prog. Nucl. Energy, 82 (2015) 33-6.

[84] E. Capelli, O. Benes, R. J. M. Konings, J. Nucl. Mater., 501 (2018) 238-52.

[85] O. Benes, R. J. M. Konings, J. Nucl. Mater., 377 (3) (2008) 449-57.

[86] O. Benes, R. J. M. Konings, CALPHAD, 32 (1) (2008) 121-8.

[87] M. Beilmann, O. Benes, R. J. M. Konings, T. Fanghanel, J. Chem. Thermodyn., 43 (10) (2011) 151524.

[88] J. A. Ocadiz-Flores, E. Capelli, P. E. Raison, R. J. M. Konings, A. L. Smith, J. Chem. Thermodyn., 121 (2018) 17-26.

[89] E. Capelli, R. J. M. Konings, J. Fluorine Chem., 208 (2018) 55-64.

[90] A. L. Smith, C. Gueneau, J. L. Fleche, S. Chatain, O. Benes, R. J. M. Konings, J. Chem. Thermodyn., 114 (2017) 93-115.

[91] P. Chartrand, A. D. Pelton, Canadian metallurgical quarterly, 39 (4) (2000) 405-20.

[92] M. Piro, J. Banfield, K. T. Clarno, S. Simunovic, T. M. Besmann, B. Lewis, W. Thompson, J. Nucl. Mater., 441 (1-3) (2013) 240-51.

[93] M. Piro, S. Simunovic, Comput. Mater. Sci., 118 (2016) 87-96.

[94] M. H. Piro, S. Simunovic, T. M. Besmann, Thermochimica User Manual v1. 0, Oak Ridge National Laboratory (ORNL), (2012)

[95] M. Piro, Adding the Capability to Solve the Modified Quasichemical Model in Thermochimica for Molten Salts, (in preperation)

[96] M. Piro, S. Simunovic, T. M. Besmann, B. Lewis, W. Thompson, Comput. Mater. Sci., 67 (2013) 266-72.

[97] I. Gauld, O. Hermann, R. Westfall, ORNL/TM-2005/39, Version, 6 (2009)

[98] R. Salko, M. Avramova, COBRA-TF Subchannel Consortium for Advanced Thermal-Hydraulics Code Simulation of LWRs (CTF) Theory Manual; 2015.

[99] M. Kurata, T. Besmann, P. Turchi, J. Dumas, E. Corcoran, M. Piro, T. Ogata, R. Hania, B. Lee, R. Kennedy,

[100] M. Rosenthal, R. Briggs, P. Haubenreich, al. Molten salt reactor program semiannual progress report for period ending august 31, ORNL-4622. USA: Oak Ridge National Laboratory, 1970: 38-41, (1968)

[101] M. Bredig, Mixtures of metals with molten salts, Oak Ridge National Lab., Tenn., (1963) 
APPENDIX A. 


\section{APPENDIX A. DEMONSTRATION OF IMPLEMENTATION OF SPECIES TRANSPORT USING COBRA-TF}

A number of sample problems were created to test the added capabilities. These include testing volumetric source term generation and depletion and convective transport problems for a number of flow directions. Figure 1 shows the results from a sample problem for volumetric source term generation of a coupled system of ${ }^{135} \mathrm{Xe}$ and ${ }^{135} \mathrm{I}$ using a constant neutron flux. This is a single channel time dependent system with no flow. Figure 1 shows CTF converges to the analytical solution with a reduction in time step size.
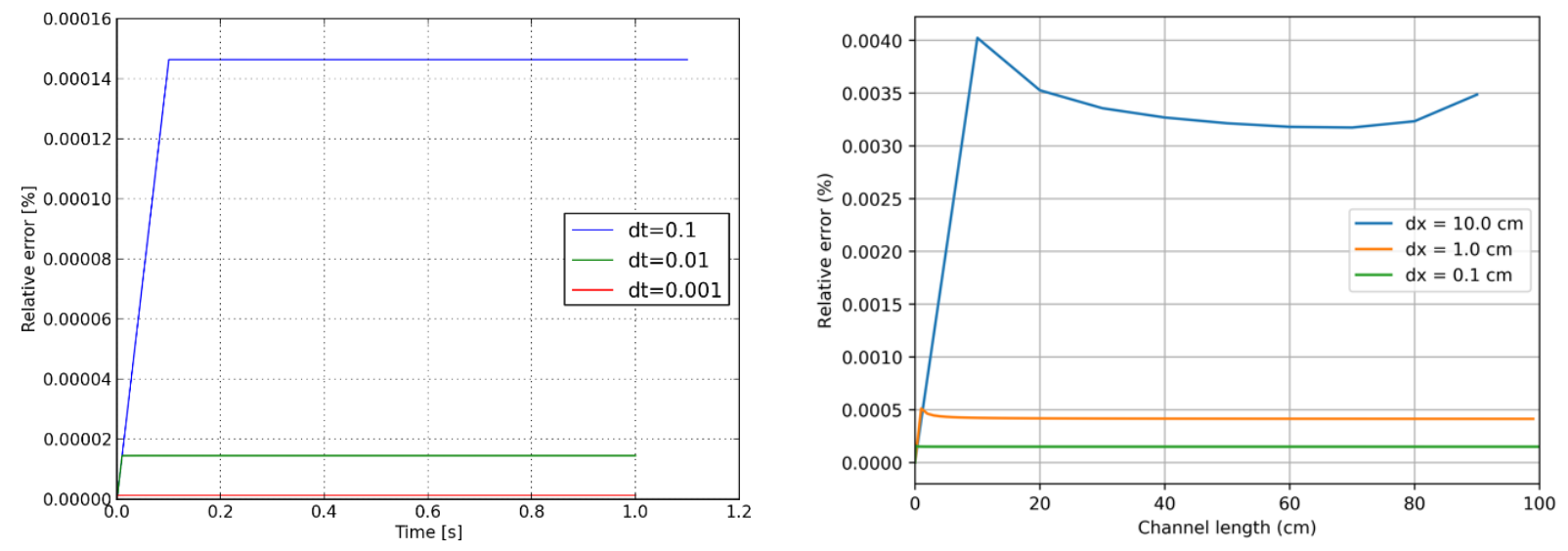

Figure 25 Relative error numerical vs analytical solution for various time steps [2]

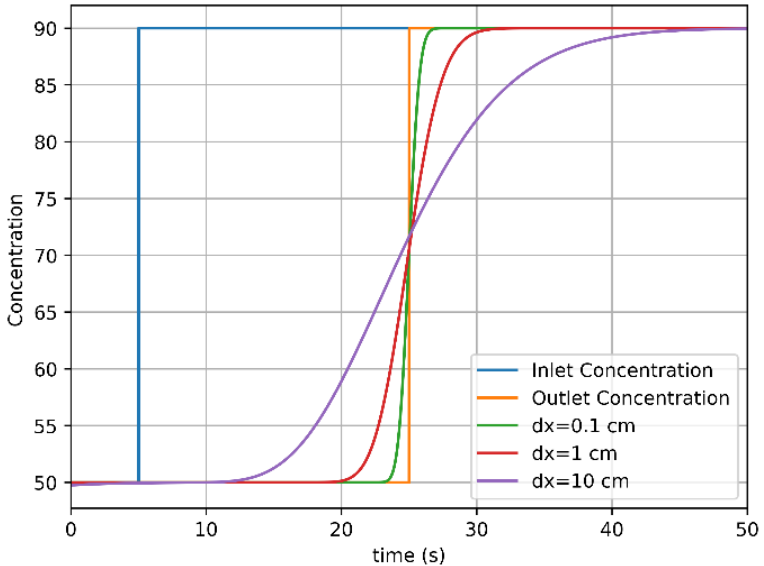

Figure 27 Step change in ${ }^{135} I$ concentration for various axial meshes [2]
Figure 26 Relative error in ${ }^{135} I$ concentration for various axial meshes [2]

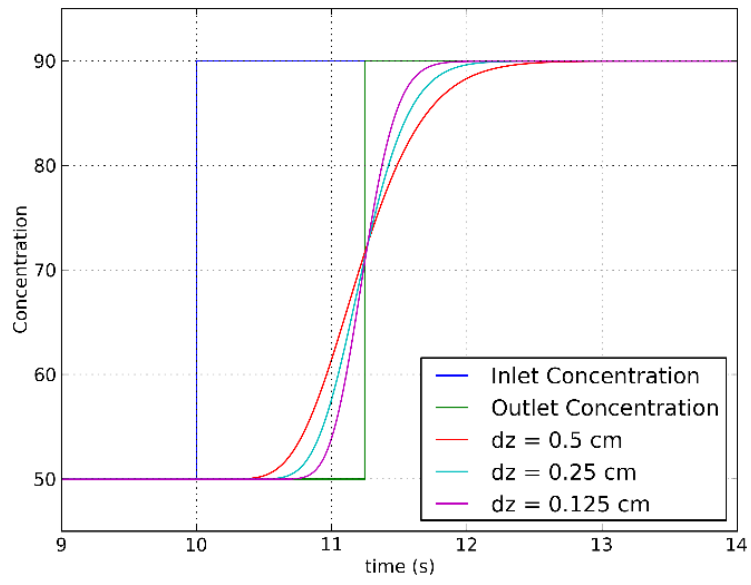

Figure 28 Step change in ${ }^{135} I$ concentration for various lateral meshes [2]

Figure 26 and Figure 27 how results for single channel flow in the $\mathrm{x}$ direction. For both problems, the fluid velocity and density were held constant. Figure 26 has a variable neutron flux applied in the $x$ direction and Figure 27has a step change in inlet concentration. Figure 28 shows results for a step change 
in concentration for a lateral flow problem ( $\mathrm{y}, \mathrm{z}$ directions). For a more detailed description of the previously mentioned test problems please refer to [2]. 
APPENDIX B. 


\section{APPENDIX B. THERMOCHEMICAL DATABASE FORMAT}

\section{The Basic Structure of a Data-File (.DAT file)}

A system is said to be assessed when sufficient experimental and theoretical data are available to optimize the adjustable parameters of each model representing each phase in that system. The models are mere smoothed continuous representations of the actual data. Computational thermodynamic (CT) software packages, like FactSage [77] or Thermocalc [67], provide a way to effectively and efficient call that data to predict equilibrium behavior. Both have wide use within the scientific community. However, Factsage is the only commercial CT code capable of handling the MQM. Further, the thermochemical equilibrium solver Thermochemica [96] for coupling to multi-physics platforms, relies on the FactSage thermochemical software suite [76] data structure written as a .DAT file. The format and models are specific to expected materials related to MSRs, namely molten and crystalline salts and the gas phase. Models for other materials, such as salt solid solution phases and metallic alloys can be included as well. These are not considered in the descriptions below.

For the purposes of the current description of a data-file, a limited example for the Li-U-F system was generated in FactSage and the software used to create the ASCII Chemsage file that appears in the APPENDIX A of this section. Note that FactSage data-files are not directly readable or editable, and their content can only be viewed and manipulated using modules of the FactSage software.

\section{Example Li-U-F system Chemsage Data-File (.DAT file)}

The header section is common to all data files. Details of the format are provided below.

\section{The header section}

Line 1 Descriptive title

The title may be up to $\mathbf{7 8}$ characters long and can be used to provide a brief description of, for example, the contents of the data-file and the source of the data. The title below describes the data-file LBUF.DAT which considers a limited Li-Be-U-F system.

Data and models for the LiF-UF4 system

Line 2 Defining the size of the data-file.

First value: Number of system components

Second value: Number of mixture phases (in this case the gaseous a liquid phase)

Third: Sequence of values for each mixture specifying number of components in the mixture in the sequence.

Last value: Number of stoichiometric condensed phases.

Note: The gas phase must always be considered as mixture phase number 1 .

Note: If a gas phase is not present in the data-file, the number of its components must still be included, and set to 0 .

For this example, system the line contains

\section{B-1}




$\begin{array}{lllll}3 & 2 & 7 & 3 & 9\end{array}$

Line 3 List of components in the system.

The names of the components are listed, as shown. Each string must consist of 25 characters, including empty spaces.

Note: If more than three components are contained in the system, extra lines must be used.

U

F

Li

Line 4 Molecular weights of the system components.

238.02891000

18.99840320

6.94100000

Line 5 Specifying Gibbs energy data format.

Code for the temperature dependence of the Gibbs energy equations for the species in the system. (Code values for the various Gibbs energy expressions are found in APPENDIX B.) These equations contain up to twelve terms of which the first six terms are predefined. The remaining six terms may be freely defined by the user.

$$
\begin{array}{r}
\mathrm{G}=\mathrm{A}+\mathrm{B} \mathrm{T}+\mathrm{C} \mathrm{T} \ln \mathrm{T}+\mathrm{D} \mathrm{T}^{2}+\mathrm{ET}^{3}+\mathrm{F} / \mathrm{T}+ \\
\mathrm{G} \mathrm{T}^{\mathrm{i}}+\mathrm{H} \mathrm{T}^{\mathrm{j}}+\mathrm{I} \mathrm{T}^{\mathrm{k}}+\mathrm{J} \mathrm{T}^{1}+\mathrm{K} \mathrm{T}^{\mathrm{m}}+\mathrm{L} \mathrm{T}^{\mathrm{n}}
\end{array}
$$

The powers in the additional terms may have any real value. However, a value of " 99 " is used as flag for "ln $\mathrm{T}$ ".

Note: It is permissible to use less than 6 terms. In this case the terms used must be chosen among the first 6 terms.

Note: If extra terms are used the total number of these terms may be between 1 and 6 . The first 6 terms as defined above are then used as the default for the entire file while the extra terms are entered with the actual substance data. Thus, each substance can have its own extra terms. Since the number of extra terms has to be entered for each temperature of a substance dataset it is even possible to choose a different number of extra terms for each temperature range.

The entry is number of terms followed by the positions in the Gibbs energy equation, i.e.,

$$
G(T)=A+B T+C T \ln T+D T^{2}+E T^{3}+F / T
$$

\section{6}

Line 6 Temperature and pressure terms.

Definition of the temperature and (if applicable) pressure dependence terms for the concentration coefficients in the excess Gibbs energy equation,

$$
L(T, P)=A+B T+C T \ln T+D T^{2}+E T^{3}+F / T+G P+H P^{2}
$$

\section{B-2}


Any number of the seven terms in the equation may be used. The entry in the data-file consists of the total number of terms defined, and their positions in the Gibbs energy equation. For the example of the LBUF.DAT file the values are the same as for the Gibbs energy equation,

\section{6}

\section{Data block for mixture phase(s)}

Data are entered for all ' $n$ ' mixture phases included in the system in any order sequence, except that the gas phase must always be considered as the first mixture phase. If a gas phase is not present, the number of its components must be set to 0 . Data for each component of a mixture phase are entered successively, as illustrated below.

\section{Data block for mixture phase 1 - the gas phase}

Entry 1: The phase name

GAS

Entry 2: The model name - IDMX (ideal mixing)

IDMX

Note that data for excess model concentration coefficients are usually entered after the last data entry for a phase component. These contributions are not present in the case of ideal mixing (IDMX).

Data for component 1 of mixture phase 1 - lithium fluoride gas

Entry 3: The component name

LiF

Entry 4: Thermodynamic data option for component 1.

First value specifies type of entered Gibbs energy data. In the example it is " 1 ," which specifies the "fixed-term Gibbs energies" format.

Second value: Number of Gibbs energy equations (to fit behavior it may be necessary to have equations for multiple temperature ranges).

Remaining values: Coefficients of the stoichiometry matrix. These are entered in the order defined in line 3 of the header. (For example, for "LiF" it is " 0.01 .01 .0 " as the stoichiometry for " $\mathrm{U}$ " is zero, and of course "Li" and " $F$ " are unity, "1.0."
11
0.0
1.0
1.0 
Note: For the "extended-term Gibbs energies" format where greater than the standard 6 terms are needed, an integer value ahead of the last line of each temperature range coefficients indicates how many terms beyond the standard 6 terms will be used, and that is followed by the values of the coefficient and the power to which temperature is raised. Again, "99" is used as flag for "ln T."

Entry 5: Specification of Gibbs energy coefficients temperature range and values of coefficients.

The upper temperature limit $(\mathrm{K})$ and the temperature coefficients for each term in the "fixedterm" Gibbs energy equation.

Note: The first value is the upper limit in Kelvin of the temperature range for the expression. The first set of values are always assumed to be valid from $298 \mathrm{~K}$ to the indicated temperature. So, the example above is valid from $298 \mathrm{~K}$ to $6000 \mathrm{~K}$ (In this example there is only one temperature range for $\operatorname{LiF}(\mathrm{g})$.)

$$
\mathrm{G}=\mathrm{A}+\mathrm{B} \mathrm{T}+\mathrm{C} \mathrm{T} \ln \mathrm{T}+\mathrm{D} \mathrm{T}^{2}+\mathrm{ET}^{3}+\mathrm{F} / \mathrm{T}
$$

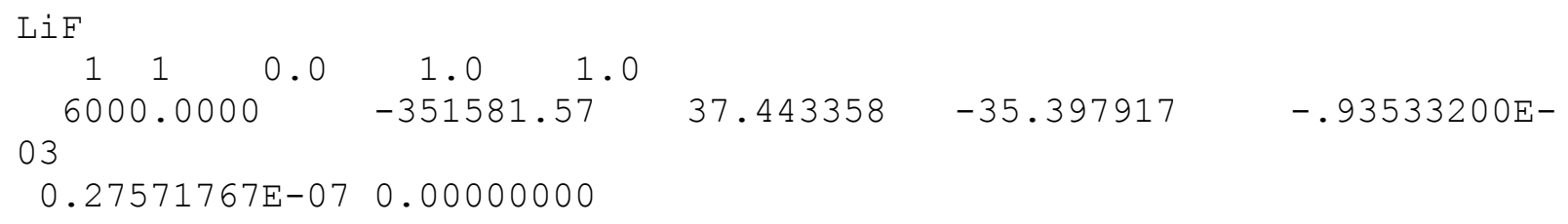

Data block for mixture phase 2 - the liquid phase

Entry 1: The phase name

LIQUSOln

Entry 2: The model name - SUBG (Modified Quasi-Chemical Model in the Quadruplet Approximation)

SUBG

Entry 3: Value of zeta.

2.4

Entry 4: Values for number of species and number of pairs/quadruplets.

23

Entry 5: The component name.

For the SUBG designated model it follows the convention of associating the relevant components, in this case Li and F, utilizing the data for LiF liquid.

$\mathrm{Li} / / \mathrm{F}$ 
Entry 6: Thermodynamic data

Values for the component following the format described above for gaseous species, again in this case values for LiF liquid.

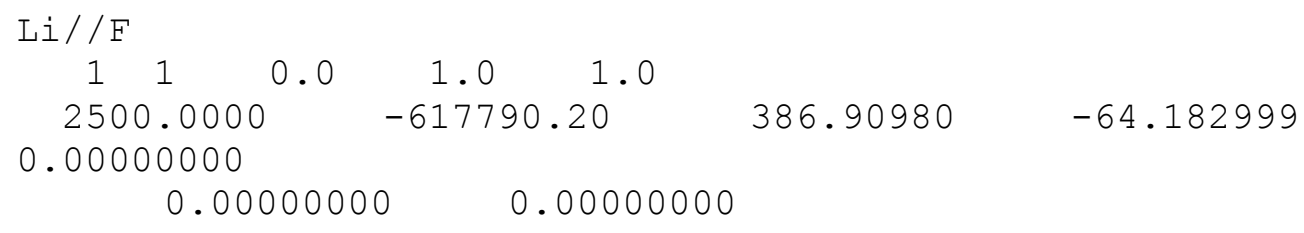

Entry 7: The number of cations and anions in the phase constituent.
1.00000
1.00000
0.000000
0.000000
0.000000

Entry 8: After completing entry of all the phase constituents within the solution, the data block specifies the number of components on each of the sublattices.

$$
21
$$

Entry 9: The components of the solution phase (cations line followed by anions line).

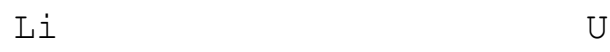

F

Entry 10: Charges on the cationic species
1.00000
4.00000

Entry 11: Cation position in listing (Li U)

12

Entry 12: Charges on the anionic species

1.00000

Entry 13: Cation position in listing (F)

1

Entry 14: Matrix to relating the phase components to the sublattice components

Each column pertains to one of the phase components, each line to one of the two sublattices. That is

Li U

F F

is specified as 
Entry 15: The four integer values ahead of the coordination numbers are the indices that specify the

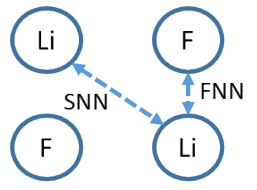
"quadruplets" representing coordination/interactions (FNN=first nearest neighbor; $\mathrm{SNN}=$ second nearest neighbor). Thus 1133 is for Li-F-Li-F, or visually in the image to the left. The remaining values in the line are the redundant coordination numbers for $\mathrm{Li}$ and $\mathrm{F}$ (6.0 for each). The two lines that follow are then obviously for U-FU-F and Li-F-U-F quadruplets.

Constituent indices followed by exponents on pair fractions.

G $1 \begin{array}{lllllllll}1 & 2 & 3 & 0 & 0 & 0 & 0\end{array}$

$\begin{array}{llllllll}1 & 1 & 3 & 3 & 6.0000000 & 6.0000000 & 6.0000000 & 6.0000000 \\ 2 & 2 & 3 & 3 & 6.0000000 & 6.0000000 & 1.5000000 & 1.5000000 \\ 1 & 2 & 3 & 3 & 2.0000000 & 6.0000000 & 1.7142857 & 1.7142857\end{array}$

The coordination numbers must follow the constraint

$$
q(i) / Z(i)+q(j) / Z(j)=q(x) / Z(x)+q(y) / Z(y)
$$

where $\mathrm{q}$ is the ionic charge and $\mathrm{Z}$ is the $\mathrm{SNN}$ coordination number. This thus results in the odd value of 1.7142857 for the Li-F-U-F quadruplet.

$$
1 / 2.00+4 / 6.00=1 / 1.7142857+1 / 1.7142857
$$

Entry 16: The excess Gibbs energies from the quadruplet-based (pair-wise) interactions are illustrated in the first set of values in the example data-file.

3

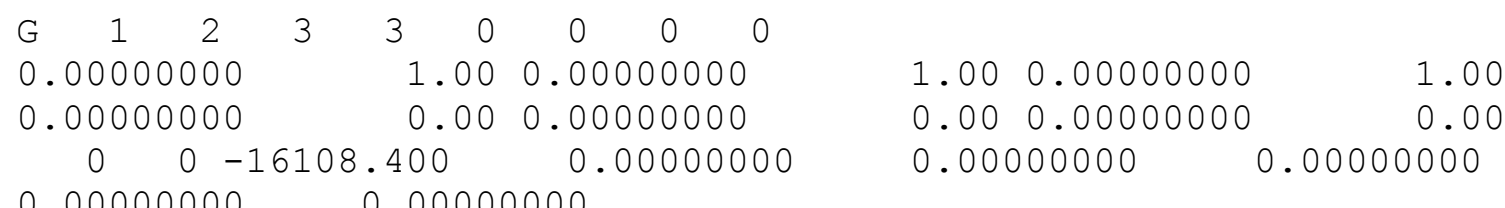

$0.00000000 \quad 0.00000000$

The first line has the value " 3 " indicating that the Gibbs energy expression uses three lines. The next line contains the letter " $G$ " to indicate a Gibbs energy expression, the following four integer values in the first line are the indices for the components $\mathrm{Li} \mathrm{U} \mathrm{F} \mathrm{F.} \mathrm{The} \mathrm{next} \mathrm{four} \mathrm{values} \mathrm{in} \mathrm{the} \mathrm{line} \mathrm{are} \mathrm{exponent} \mathrm{values} \mathrm{on}$ the site fractions, the first being $X(\mathrm{Li} \mathrm{Li} \mathrm{F} \mathrm{F)}$, next X(U U F F), then $\mathrm{X}(\mathrm{U}$ Li F F) and X ( $\mathrm{Li} \mathrm{U} \mathrm{F} \mathrm{F).} \mathrm{In}$ this case they are all zero, although in the second and third $\mathrm{G}$ expressions they have values of 1 for two of the site fractions.

The values for the Gibbs energy expression actually are only usefully found on the third line (which extends to a fourth line in the text, but in the code is the extended third line), with the first integer corresponding to the constituent index number for any ternary mixing terms. The second integer term is, provisionally, the exponent on the mixing site fraction. (If writing a file, use the values in the lines as shown for the first and second Gibbs energy expression.) The remaining values follow the standard Gibbs expression 


$$
\mathrm{G}=\mathrm{A}+\mathrm{B} \mathrm{T}+\mathrm{C} \mathrm{T} \ln \mathrm{T}+\mathrm{D} \mathrm{T}^{2}+\mathrm{ET}^{3}+\mathrm{F} / \mathrm{T}
$$

The last line for the series of solutions displays the integer value zero.

\section{Data block for stoichiometric compounds}

The Gibbs energy expressions for all the stoichiometric compounds are listed last in the data-file. Below is an example

\begin{tabular}{|c|c|c|c|}
\hline \multicolumn{4}{|c|}{$110 \mathrm{n}+10$} \\
\hline $\begin{array}{ccc}1 & 1 & 0.0 \\
2500.0000\end{array}$ & $\begin{array}{cc}1.0 & 1.0 \\
-632481.90\end{array}$ & 262.49310 & $-43,308980$ \\
\hline $\begin{array}{l}.81560840 \mathrm{E}-02 \\
-.84117330 \mathrm{E}-07\end{array}$ & 284561.80 & & \\
\hline
\end{tabular}

The first line contains the name of the compound.

The second line contains values as follows

- Integer value designating the Gibbs energy expression format.

- Integer value indicating the number of Gibbs energy expressions that are used to span the entire temperature range of interest.

- Series of values that are the stoichiometric coefficients of the phase in the order of the systems as designated in the third line at the top of the data-file. In this case the elements are listed in the order $\mathrm{U} \mathrm{Li} \mathrm{F}$, so for $\mathrm{LiF}$ the values are 0.01 .01 .0

- The last line contains the temperature range for the expression, the temperature coefficients, and where necessary temperature exponents for "extended" the Gibbs energy expression formats.

- Note that the first value is the upper limit in Kelvin of the temperature range for the expression. The first set of values are always assumed to be valid from $298 \mathrm{~K}$ to the indicated temperature. So, the example above is valid from $298 \mathrm{~K}$ to $2500 \mathrm{~K}$.

Finally, the data-file requires the inclusion of "dummy species/phases" added for numerical stability at standard states, yet they are not appearing as stable phases in any calculations. These are designated with the symbol "\#” on first line (example below).

\begin{tabular}{|c|c|c|c|}
\hline U_Solid-A(s) & \# & & \\
\hline $\begin{array}{lll}1 & 2 & 1.0\end{array}$ & $0.0 \quad 0.0$ & & \\
\hline 942.00000 & -8407.7857 & 130.87411 & -26.919856 \\
\hline $0.12510160 \mathrm{E}-02$ & & & \\
\hline$-.44263233 E-05$ & 38492.800 & & \\
\hline 5000.0000 & -17115.788 & 247.03511 & -42.927840 \\
\hline 0.00000000 & & & \\
\hline 0.00000000 & 0.00000000 & & \\
\hline
\end{tabular}


APPENDIX C. 


\section{APPENDIX C. EXAMPLE THERMOCHEMICAL DATABASE}

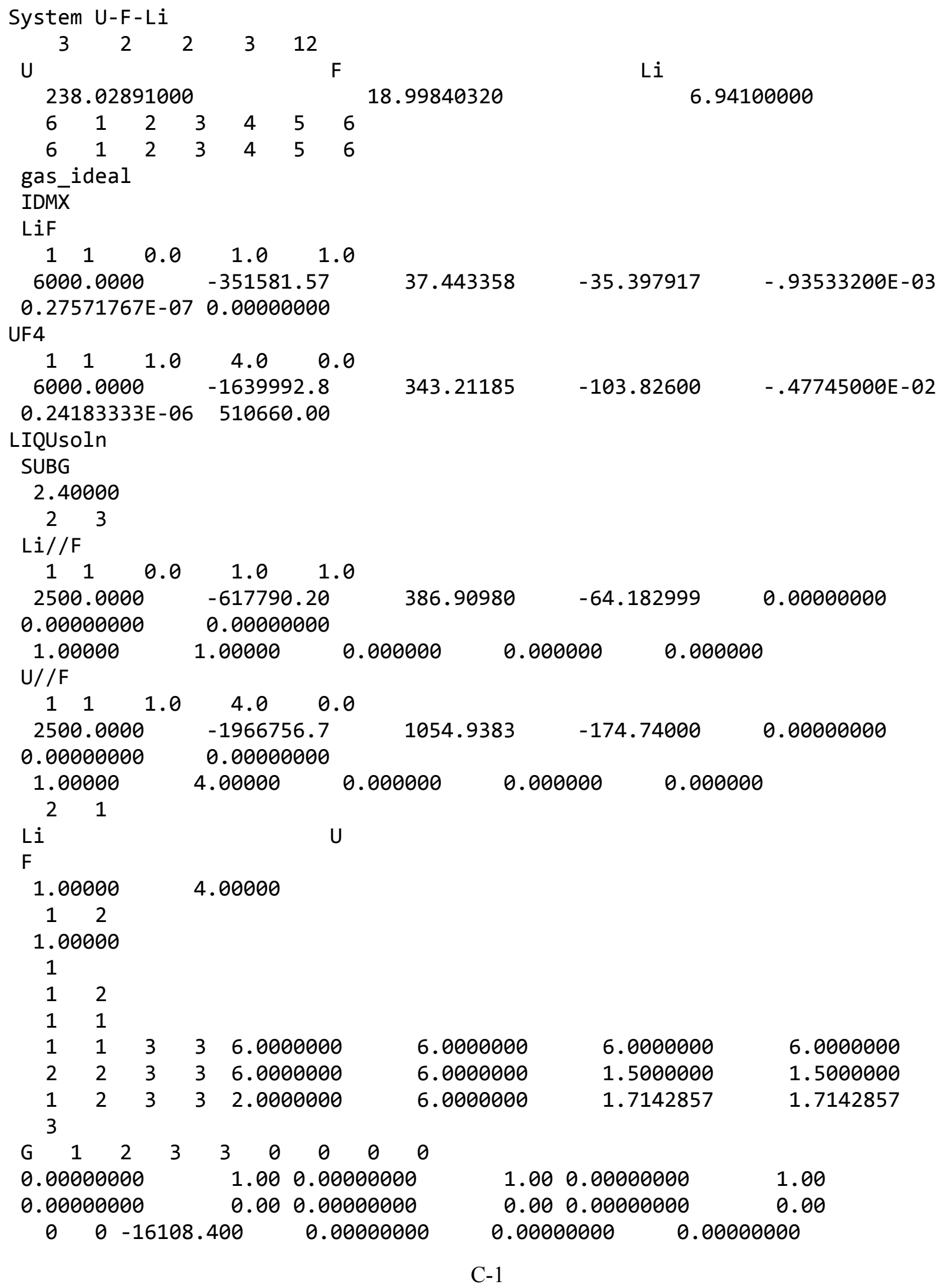




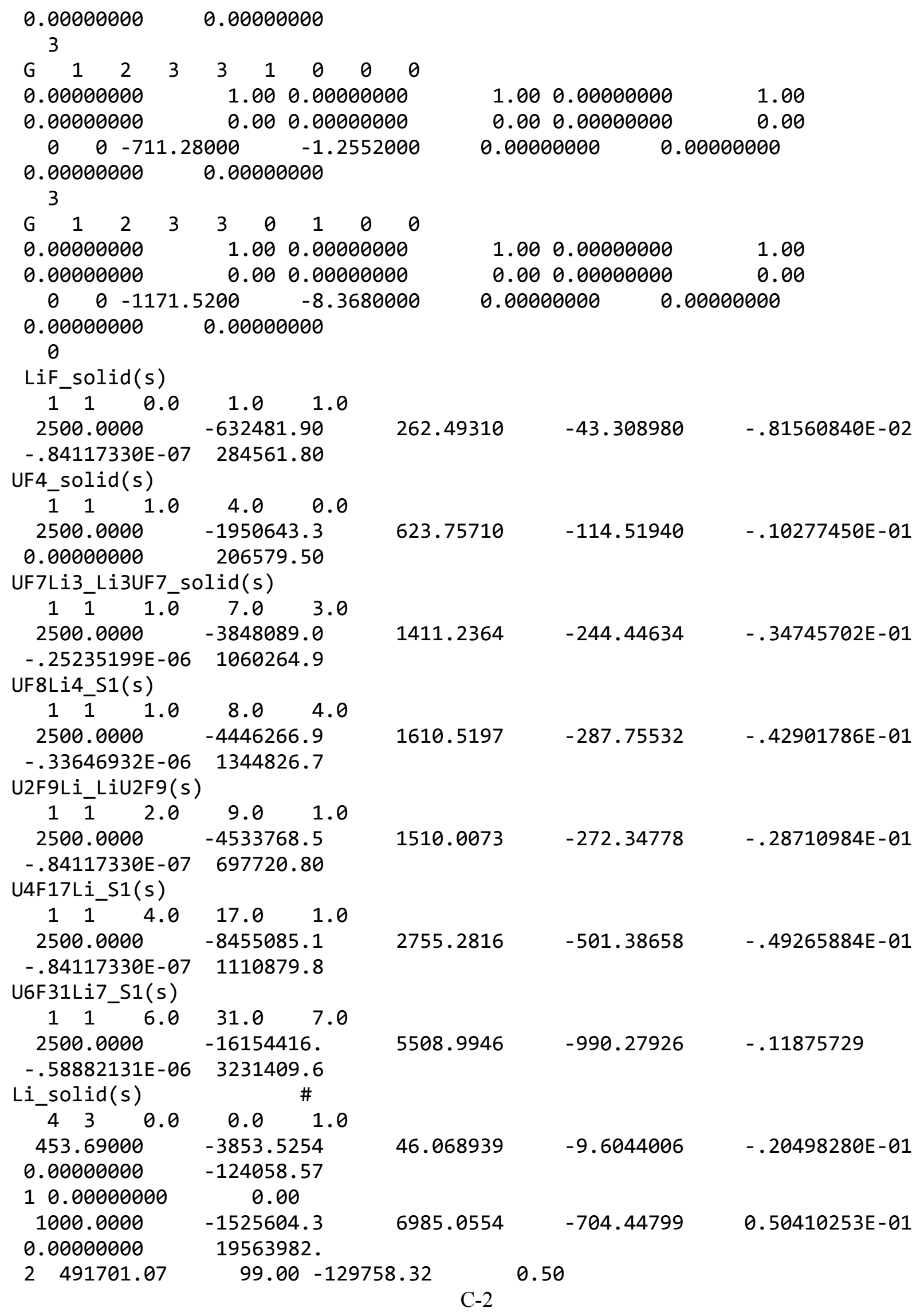




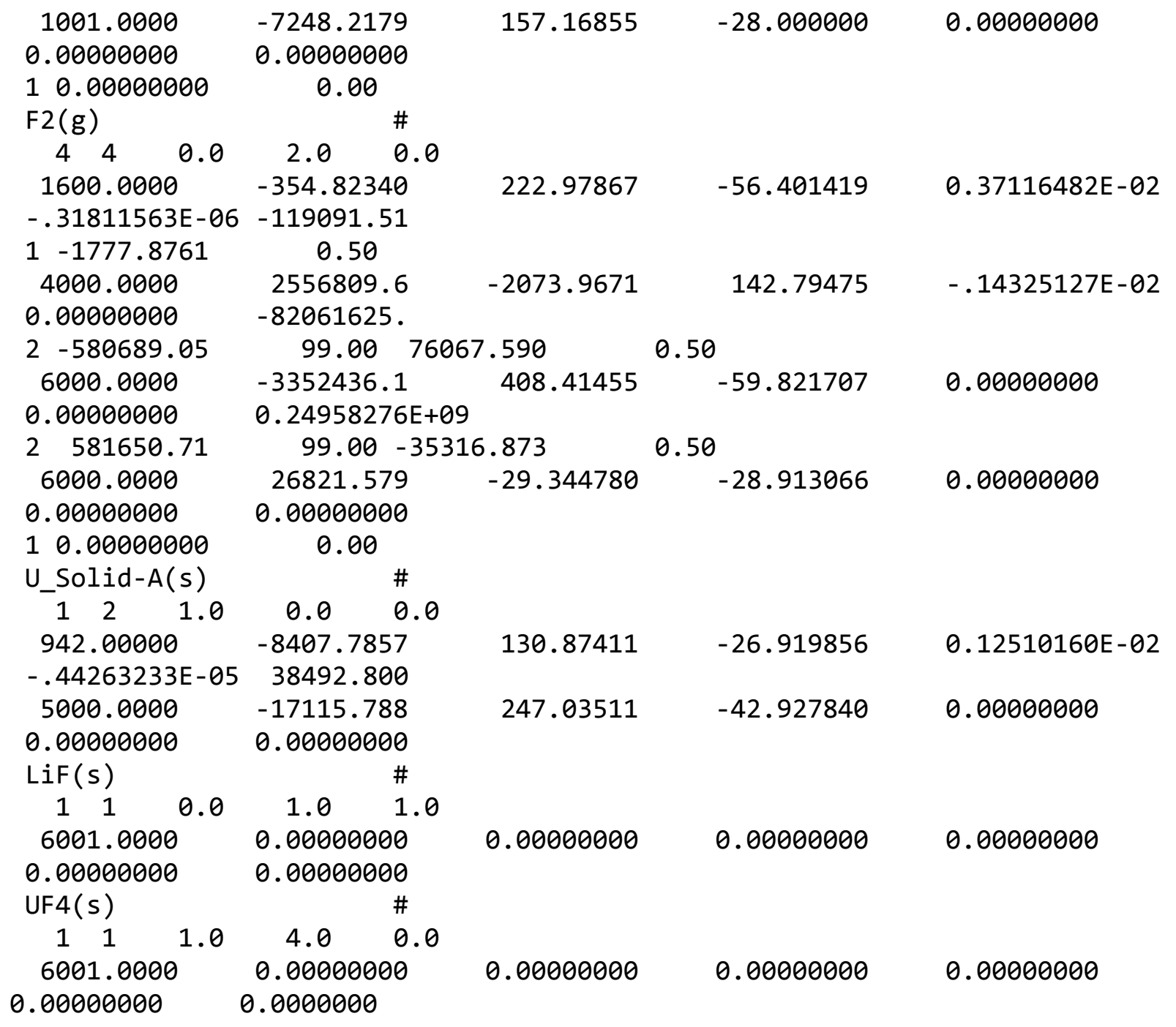


APPENDIX D. 


\section{APPENDIX D: THERMOCHEMICAL MODELS CODE VALUE KEY}

Code values for the various available expressions for the Gibbs energies

1 Fixed-term Gibbs energies

2 Fixed-term Gibbs energies, const. molar vols .

3 Fixed-term Gibbs energies, P,T-dependent molar vols .

4 Extended Gibbs energies

5 Extended Gibbs energies, const. molar vols

6 Extended Gibbs energies, P,T-dependent molar vols .

7 Enthalpy, entropy, fixed-term heat capacities

8 Enthalpy, entropy, fixed-term heat capacities, canst . molar vols.

9 Enthalpy, entropy, fixed-term heat capacities, P,T-dependent molar volumes

10 Enthalpy, entropy, extended heat capacities

11 Enthalpy, entropy, extended heat capacities, constant molar volumes

12 Enthalpy, entropy, extended heat capacities, P,T-dependent molar volumes

Add on 12 if magnetic contributions will be entered.

Fixed-term heat capacity equation $=\mathrm{a}+\mathrm{bT}+\mathrm{cT} \mathrm{T}^{2}+\mathrm{d} / \mathrm{T}^{2}$

Extended equations contain a maximum 6 additional terms with variable powers of temperature.

Gibbs energy format with the fixed-term values being the first 6 (A-F) and extended values the next $6(\mathrm{G}-\mathrm{L})$.

$$
\begin{array}{r}
\mathrm{G}=\mathrm{A}+\mathrm{B} \mathrm{T}+\mathrm{C} \mathrm{T} \ln \mathrm{T}+\mathrm{D} \mathrm{T}^{2}+\mathrm{ET}^{3}+\mathrm{F} / \mathrm{T}+ \\
\mathrm{G} \mathrm{T}^{\mathrm{i}}+\mathrm{H} \mathrm{T}^{\mathrm{j}}+\mathrm{I} \mathrm{T}^{\mathrm{k}}+\mathrm{J} \mathrm{T}^{1}+\mathrm{K} \mathrm{T}^{\mathrm{m}}+\mathrm{L} \mathrm{T}^{\mathrm{n}}
\end{array}
$$

